ARPEFS as an Analytic Technique

\author{
Alexis Eric Schach von Wittenau \\ Ph.D. Thesis \\ Materials and Chemical Sciences Division \\ Lawrence Berkeley Laboratory \\ University of California \\ Berkeley, CA 94720
}

April 1991

This work was supported by the Director, Office of Energy Research, Office of Basic Energy Sciences, Chemical Sciences Division of the U.S. Department of Energy under Contract No. DE-AC03-76SF00098. 


\title{
ARPEFS as an Analytic Technique
}

by

Alexis Eric Schach von Wittenau

\begin{abstract}
Two modifications to the ARPEFS technique are introduced. These are studied using $\mathrm{p}(2 \times 2) \mathrm{S} / \mathrm{Cu}(001)$ as a model system.

The first modification is the obtaining of ARPEFS $\chi(\mathrm{k})$ curves at tem peratures as low as our equipment will permit $(\sim 110 \mathrm{~K})$. While adding to the difficu ty of the experiment, this modification is shown to almost double the signal-to-ncise ratio of normal emission $\mathrm{p}(2 \times 2) \mathrm{S} / \mathrm{Cu}(001) \chi(\mathrm{k})$ curves. This is shown by visual comparison of the raw data and by the improved precision of the extracted structural parameters.

The second change is the replacement of manual fitting of the Fourier filtered $\chi(k)$ curves by the use of the simplex algorithm for parameter determination. Again using $\mathrm{p}(2 \times 2) \mathrm{S} / \mathrm{Cu}(001)$ data, this is shown to result in better agreement between experimental $\chi(k)$ curves and curves calculated based on model structures. The improved ARPEFS is then applied to $\mathrm{p}(2 \times 2) \mathrm{S} / \mathrm{Ni}(111)$ and $(\sqrt{3} \times \sqrt{3}) \mathrm{R} 30^{\circ} \mathrm{S} / \mathrm{Ni}(111)$.

For $\mathrm{p}(2 \times 2) \mathrm{S} / \mathrm{Cu}(001)$ we find a $\mathrm{S}-\mathrm{Cu}$ bond length of $2.26 \AA$, with the $\mathrm{S}$ adatom $1.31 \AA$ above the fourfold hollow site. The second $\mathrm{Cu}$ layer appears to be corrugated. Analysis of the $\mathrm{p}(2 \times 2) \mathrm{S} / \mathrm{Ni}(111)$ data indicates that the $\mathrm{S}$ adatom adsorbs onto the FCC threefold hollow site $1.53 \AA$ above the Ni surface. The $\mathrm{S}-\mathrm{Ni}$ bond length is determined to be $2.13 \AA$, indicating an outwards shift of the first layer $\mathrm{Ni}$ atonis. We are unable to assign a unique structure to $(\sqrt{3} \times \sqrt{3}) \mathrm{R3} 0^{\circ} \mathrm{S} / \mathrm{Ni}(111)$.
\end{abstract}


An analysis of the strengths and weaknesses of ARPEFS as an experimental and analytic technique is presented, along vith a summary of problems still to be addressed.

Finally, results of normal emission ARPES measurements of Au(001) are presented. Empirical valence band dispersion relations along the $\Gamma-\mathrm{X}$ line are determined and values of critical point energies at $\Gamma$ and $X$ are presented. 


\section{Dedication}

This thesis is dedicated to my family and especially to my parents. 


\section{Contents}

Dedication $\quad$ ii

List of Figures vi vi

List of Tables $\quad$ ix

Acknowledgements $\quad$ -

1 Introduction 1

1.1 ARPEFS, Single Scattering Approximation . . . . . . . . . 1

1.2 Figure Captions ..................... 7

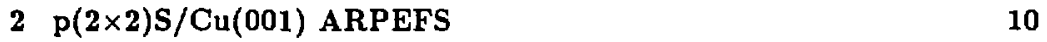

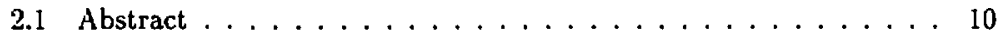

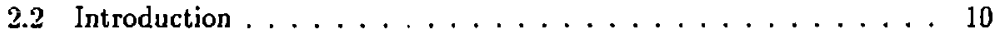

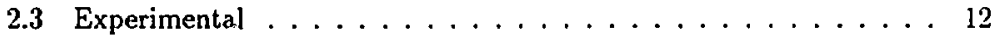

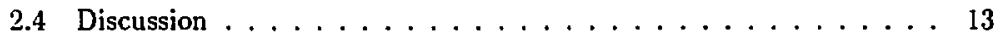

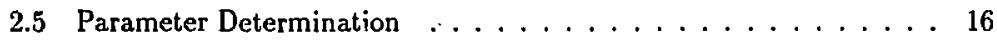

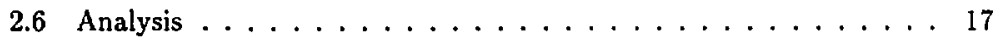

2.6.1 Normal Emission, $110 \mathrm{~K}$ and $155 \mathrm{~K} \ldots \ldots . . \ldots 17$

2.6.2 $40^{\circ}$ Off Normal Emission, $140 \mathrm{~K} \ldots \ldots . \ldots 18$

$2.6 .350^{\circ}$ Off Normal Emission, $110 \mathrm{~K} \ldots \ldots 18$ 
2.6 .4 Overview $\ldots \ldots \ldots \ldots \ldots \ldots \ldots \ldots$

2.7 Conclusion . . . . . . . . . . . . . . . . 20

2.8 Error Analysis . . . . . . . . . . . . . . . . . . 20

2.9 Comparison with Previous Work . . . . . . . . . . . . 22

2.10 Acknowledgements $\ldots \ldots \ldots \ldots \ldots \ldots$

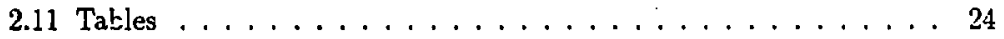

2.12 Figure Captions $\ldots \ldots \ldots \ldots \ldots \ldots \ldots \ldots \ldots$

3 S/Ni(111) ARPEFS 46

3.1 Abstract ........................ . . 46

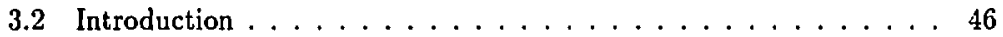

3.3 Experimental $\ldots \ldots \ldots \ldots \ldots \ldots \ldots$

3.4 Discussion $\ldots \ldots \ldots \ldots \ldots \ldots \ldots \ldots \ldots \ldots$

$3.4 .1 \mathrm{p}(2 \times 2) \mathrm{S} / \mathrm{Ni}(111) \ldots \ldots \ldots \ldots \ldots$

$3.4 .2(\sqrt{3} \times \sqrt{3}) \mathrm{R} 30^{\circ} \mathrm{S} / \mathrm{Ni}(111) \ldots \ldots \ldots \ldots \ldots$

3.4.3 Summary, $(\sqrt{3} \times \sqrt{3}) \mathrm{R30} 0^{\circ} \mathrm{S} / \mathrm{Ni}(111) \ldots \ldots \ldots \ldots$

3.5 Conclusions $\ldots \ldots \ldots \ldots \ldots \ldots \ldots \ldots \ldots \ldots \ldots \ldots \ldots \ldots \ldots$

3.6 Acknowledgements $\ldots \ldots \ldots \ldots \ldots \ldots \ldots$

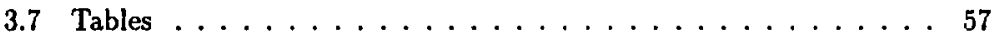

3.8 Figure Captions . . . . . . . . . . . . . . . 58

4 ARPEFS as an Analytic Technique 74

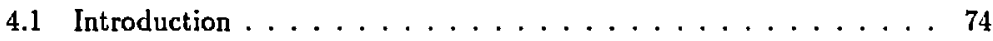

4.2 Getting the Data . . . . . . . . . . . . 74

4.2 .1 1s Spectra . . . . . . . . . . . . . 75

4.2.2 Background Spectra $\ldots \ldots \ldots \ldots \ldots$

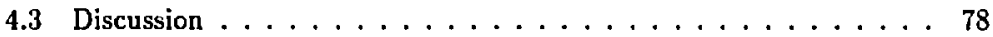


4.4 Modelling the Data $\ldots \ldots \ldots \ldots \ldots \ldots$

4.4.1 Calculating Theoretical TSMQNE Spectra . . . . . . . 81

4.4.2 Varying the Structural Parameters $\ldots \ldots \ldots \ldots$

4.4.3 Assorted Constraints . . . . . . . . . . . 86

4.5 Conclusions $\ldots \ldots \ldots \ldots \ldots \ldots \ldots \ldots$

4.6 Tables $\ldots \ldots \ldots \ldots \ldots \ldots \ldots \ldots \ldots \ldots \ldots$

4.7 Figure Captions . . . . . . . . . . . . . . 94

5 Effects of Surface Reconstruction in Valence Band Photoemission:

$\begin{array}{ll}\text { Applications to } \mathrm{Au}(001) & 108\end{array}$

5.1 Abstract . . . . . . . . . . . . . . . . 108

5.2 Introduction $\ldots \ldots \ldots \ldots \ldots \ldots \ldots \ldots \ldots \ldots \ldots$

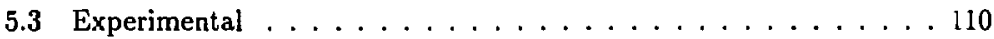

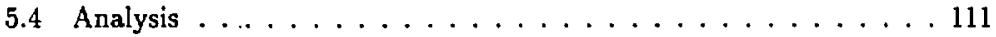

5.4 .1 Selection Rules $\ldots \ldots \ldots \ldots \ldots \ldots \ldots$

5.4 .2 Valence Band Structure $\ldots \ldots \ldots \ldots . \ldots 112$

5.4 .3 Critical Point Energies . . . . . . . . . . . . 114

5.5 Umklapp Effects . . . . . . . . . . . . . . . . 114

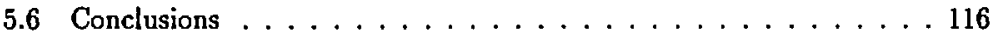

5.7 Acknowledgements $\ldots \ldots \ldots \ldots \ldots$

5.8 Table . . . . . . . . . . . . . . . . . . . 118

5.9 Figure Captions $\ldots \ldots \ldots \ldots \ldots \ldots$

6 Conclusions $\quad 128$

6.1 Table ........................ . 131

$\begin{array}{ll}\text { References } & 132\end{array}$ 


\section{List of Figures}

1.1 Schematic of the ARPEFS Process $\ldots \ldots \ldots \ldots \ldots$

1.2 Flowchart of ARPEFS Experiments . . . . . . . . . . 9

$2.1 \mathrm{p}(2 \times 2) \mathrm{S} / \mathrm{Cu}(001)$ Structure $\ldots \ldots \ldots \ldots \ldots \ldots . \ldots . \ldots . \ldots 2$

$2.2 \mathrm{p}(2 \times 2) \mathrm{S} / \mathrm{Cu}(001)$ Raw Data . . . . . . . . . . . 33

2.3 Fits to $110 \mathrm{~K}$ Normal Emission Data, $\mathrm{p}(2 \times 2) \mathrm{S} / \mathrm{Cu}(001) \ldots \ldots .34$

2.4 Fits to $155 \mathrm{~K}$ Normal Emission Data, $\mathrm{p}(2 \times 2) \mathrm{S} / \mathrm{Cu}(001) \ldots \ldots . .35$

2.5 Fits to $40^{\circ}$ Off Normal Emission Data, $\mathrm{p}(2 \times 2) \mathrm{S} / \mathrm{Cu}(001) \ldots 36$

2.6 Fits to $50^{\circ}$ Off Normal Emission Data, $\mathrm{p}(2 \times 2) \mathrm{S} / \mathrm{Cu}(001) \ldots 37$

2.7 R-Factor vs. $\mathrm{D}_{\mathrm{s} 1}, \mathrm{p}(2 \times 2) \mathrm{S} / \mathrm{Cu}(001) \ldots \ldots \ldots . \ldots 38$

2.8 R-Factor vs. $\mathrm{D}_{\mathrm{Sc}}, \mathrm{p}(2 \times 2) \mathrm{S} / \mathrm{Cu}(001) \ldots \ldots \ldots \ldots$

2.9 R-Factor vs. $\mathrm{D}_{\mathrm{so}}, \mathrm{p}(2 \times 2) \mathrm{S} / \mathrm{Cu}(001) \ldots \ldots \ldots \ldots$

2.10 R-Factor vs. $\mathrm{D}_{\mathrm{SA}}, \mathrm{p}(2 \times 2) \mathrm{S} / \mathrm{Cu}(001) \ldots \ldots \ldots \ldots \ldots \ldots$

2.11 R-Factor vs. $\mathrm{D}_{\mathrm{S} 3}, \mathrm{p}(2 \times 2) \mathrm{S} / \mathrm{Cu}(001) \ldots \ldots \ldots \ldots \ldots \ldots$

2.12 R-Factor vs. S-Cu Bond Length, $\mathrm{p}(2 \times 2) \mathrm{S} / \mathrm{Cu}(001) \ldots \ldots \ldots$

2.13 Effect of Temperature on $\chi$ Oscillations, $\mathrm{p}(2 \times 2) \mathrm{S} / \mathrm{Cu}(001) \ldots 44$

2.14 Refit of $300 \mathrm{~K}$ Normal Emission Data, $\mathrm{p}(2 \times 2) \mathrm{S} / \mathrm{Cu}(001) \ldots . .45$

$3.1 \mathrm{p}(2 \times 2) \mathrm{S} / \mathrm{Ni}(111)$ Structure $\ldots \ldots \ldots \ldots \ldots . \ldots \ldots 2$

3.2 Raw $\mathrm{p}(2 \times 2) \mathrm{S} / \mathrm{Ni}(111)$ Data $\ldots \ldots \ldots \ldots \ldots 63$

3.3 Fits of $35^{\circ}$ Off Normal $\mathrm{p}(2 \times 2) \mathrm{S} / \mathrm{Ni}(111)$ Data $\ldots \ldots \ldots 64$ 
3.4 Fits of $50^{\circ}$ Off Normal $\mathrm{p}(2 \times 2) \mathrm{S} / \mathrm{Ni}(111)$ Data . . . . . . . . 65

3.5 R-Factor vs. Structural Parameters, $\mathrm{p}(2 \times 2) \mathrm{S} / \mathrm{Ni}(111) \ldots \ldots 66$

3.6 Raw Data, $(\sqrt{3} \times \sqrt{3}) \mathrm{R} 30^{\circ} \mathrm{S} / \mathrm{Ni}(111) \ldots \ldots \ldots \ldots \ldots$

3.7 Comparison of $(\sqrt{3} \times \sqrt{3}) \mathrm{R} 30^{\circ} \mathrm{S} / \mathrm{Ni}(111)$ Normal Emission Data with 3-Fold Hollow Sites $\ldots \ldots \ldots \ldots$. . . . . . 68

3.8 Comparison of $(\sqrt{3} \times \sqrt{3}) \mathrm{R} 30^{\circ} \mathrm{S} / \mathrm{Ni}(111) 35^{\circ}$ Off Normal Emission Data with 3-Fold Hollow Sites . . . . . . . . . . . . . . . 69

3.9 Comparison of $(\sqrt{3} \times \sqrt{3}) \mathrm{R} 30^{\circ} \mathrm{S} / \mathrm{Ni}(111) 50^{\circ}$ Off Normal Emission Data with 3-Fold Hollow Sites . . . . . . . . . . . . . 70

3.10 Comparison of $(\sqrt{3} \times \sqrt{3}) \mathrm{R} 30^{\circ} \mathrm{S} / \mathrm{Ni}(111)$ Normal Emission Data with Bridged and Atop Sites . . . . . . . . . . . 71

3.11 Comparison of $(\sqrt{3} \times \sqrt{3}) \mathrm{R} 30^{\circ} \mathrm{S} / \mathrm{Ni}(111) 35^{\circ}$ Off Normal Emission Data with Bridged and Atop Sites . . . . . . . . . . 72

3.12 Comparison of $(\sqrt{3} \times \sqrt{3}) \mathrm{R} 30^{\circ} \mathrm{S} / \mathrm{Ni}(111) 50^{\circ}$ Off Normal Emission Data with Bridged and Atop Sites . . . . . . . . . . . 73

4.1 Typicai 1s Spectrum with Fitting Functions . . . . . . . . 98

4.2 Normalization of Individual 1s Spectra . . . . . . . . . . . 99

4.3 Typical Background Spectrum . . . . . . . . . . . . . 100

4.4 Reproducibility of Curves Taken at Different Beamlines . . . . . . 101

4.5 Difference in statistics for 1 s spectra used for $\mathrm{I}(\mathrm{E})$ curves $\ldots \ldots . .102$

4.6 Effects of Count Rate Saturation . . . . . . . . . . . 103

4.7 Possible Path Length Difference Cutoffs . . . . . . . . . . . 104

4.8 Different forms of the inelastic mean free path in $\mathrm{Cu} \ldots \ldots \ldots 105$

4.9 Importance of scattering atoms to the ARPEFS, normal emission $\mathrm{p}(2 \times 2) \mathrm{S} / \mathrm{Cu}(001) \ldots \ldots \ldots \ldots \ldots \ldots$ 
4.10 Importance of scattering atoms to the ARPEFS, $40^{\circ}$ off normal emission $\mathrm{p}(2 \times 2) \mathrm{S} / \mathrm{Cu}(001) \ldots \ldots \ldots \ldots$. . . . . . . . . 107

5.1 Au(001) Raw Data . . . . . . . . . . . . . . . . 121

5.2 Theoretical Au Valence Bands . . . . . . . . . . . . . 123

5.3 Empirical Band Maps . . . . . . . . . . . . . . . . . . . 124

5.4 Used Portions of Conduction Bands . . . . . . . . . . 125

5.5 Surface Umklapp Bands $\ldots \ldots \ldots \ldots \ldots \ldots$

5.6 Surface Umklapp Features . . . . . . . . . . . . . . . 127 


\section{List of Tables}

2.1 Literature Structures of $\mathrm{p}(2 \times 2) \mathrm{3} / \mathrm{Cu}(001) \ldots \ldots \ldots \ldots 24$

2.2 ARPEFS $\mathrm{p}(2 \times 2) \mathrm{S} / \mathrm{Cu}(001)$ Strucuure, $k$-Space Fits $\ldots \ldots \ldots 25$

2.3 ARPEFS $\mathrm{p}(2 \times 2) \mathrm{S} / \mathrm{Cu}(001)$ Structure, R-Space Fits . . . . . . 25

2.4 ARPEFS $\mathrm{p}(2 \times 2) \mathrm{S} / \mathrm{Cu}(001)$ Structure, Average Values $\ldots \ldots .25$

$2.5 \mathrm{p}(2 \times 2) \mathrm{S} / \mathrm{Cu}(001)$ Structure, Refit of Previous Data . . . . . . 26

3.1 Literature Structures of $\mathrm{p}(2 \times 2) \mathrm{S} / \mathrm{Ni}(111) \ldots \ldots \ldots \ldots \ldots 57$

3.2 ARPEFS Structure of $\mathrm{p}(2 \times 2) \mathrm{S} / \mathrm{Ni}(111) \ldots \ldots \ldots \ldots \ldots 57$

4.1 k-Space Fits With Different Inelastic Mean Free Path Models . . . 92

4.2 CPU Time Requirements, One Theory Curve . . . . . . . 92

4.3 CPU Time Requirements to Fit One Curve . . . . . . . 93

5.1 Critical Point Energies for Au. . . . . . . . . . . . 11s

6.1 Bond Length Comparison $\ldots \ldots \ldots \ldots \ldots \ldots \ldots \ldots 131$ 


\section{Acknowledgements}

I am deeply grateful to my family for their love, support, and faith in me.

I would like to thank many people at LBL for their help over the years. First is Dr. Michele Siggel, whose constant support, encouragement and advice in these last lew years made finishing this thesis possible. I would also like to thank Tony Moscarelli for his friendship and patient instruction in machining. Marty Gelbaum kindly answered countless programming questions. Joe Katz and Mike Press did likewise for electronics questions.

Within the Shirley group I would like to thank Wini Heppler for instruction in crystal preparation and Laue diffraction, and for polishing the many crystals she did. The wine-making and smoked turkey dinners were very much appres: ited. Barbara Moriguchi handled the paperwork needed for the various synchrotron runs and to keep the group operating smoothly.

Jim Tobin and Charlie Bahr got me started in experimental and computer worl. For invaluable help on other runs and work I have to tlıank Drs. Zahid Hussain, K. Tong Leung, Louis Terminello, Sehun Kim, and Xun-Sheng Zliang.

Drs. Gunter Kaindl, Bill Brewer, and Gerhard Kalkowski of the Free University of Berlin introduced the joys of dedicated running on Jumbo to me. Jane Medhurst taught me about the grasshopper.

Changqin Xue and Guido Remmers got me set up on their computers at the 
Free University of Berlin, where the final ARPEFS analyses were completed.

Most of all, I would like to thank my advisor Dr. David Shirley, under whose aegis this all took place and who suggested the $\mathrm{p}(2 \times 2) \mathrm{S} / \mathrm{Cu}(001)$ problem. This triggered the analysis of the ARPEFS technique and formed the basis of this thesis. I would also like to thank him for fostering my efforts as a graduate student and for his encouraging my extensive use of the LBL computers. Through him I was able to go to the physics department of the Free University of Berlin, the first time to collaboraie with the Kaindl group on analysis of data taken at SSRL and the second time to finish analyzing my thesis data during his residency in that department.

This work was supported by the Director, Office of Energy Research, Office of Basic Energy Sciences, Chemical Sciences Division of the U.S. Department of Energy under Contract No. DE-AC03-76SF00098. 


\section{Chapter 1}

\section{Introduction}

\subsection{ARPEFS, Single Scattering Approximation}

In the case of surface studies, photoelectron diffraction is the interference between the direct outgoing wave and the scattered waves from the substrate atoms. We show a two-atom example of this in Figure 1.1. An outgoing spherical photoelectron wave (long dashes) is created when a core-electron is excited to an unbound level. A portion of this primary wave travels directly towards the detector. Another portion travels towards a substrate atom and is scattered by it. Part of this scattered wave (short dashes) the travels towards the detector. Because of the phase difference between the primary and scattered waves, they may interfere constructively or destructively. This leads to a modulation of the measured photocurrent.

Let us rephrase this in a more mathematical form. We have the primary wave

$$
\Psi_{\mathrm{P}}(\mathrm{r})=\mathrm{A} \frac{\mathrm{e}^{\mathrm{ikr}}}{\mathrm{kr}}
$$

where $\mathrm{A}=\mathrm{A}\left(k, \hat{\theta}_{e}, \hat{\phi}_{\mathrm{e}}, \hat{\mathrm{o}}_{\mathrm{h} \nu}, \hat{\phi}_{\mathrm{h} \nu}\right)$ is the angular variation in the spherical wave (e.g., a $\mathrm{p}$-wave if the initial state is an s orbital). When the primary wave reaches the scattering atom ' $\mathrm{j}$ ' at $R_{\mathrm{j}}$, we have

$$
\Psi_{P}(r)=A \frac{e^{i k R_{j}}}{k R_{j}}
$$


The primary wave undergoes a phase shift $|\mathbf{f}| \mathrm{e}^{\mathrm{i} \phi_{j}}$ as it is scattered. This scattered wave

$$
\Psi_{S}(r)=\left[A|f| \frac{e^{i k R_{i}}}{k R_{j}} e^{i \phi_{j}}\right] \frac{e^{i k\left(r-R_{i}\right)}}{k\left(r-R_{j}\right)}
$$

then travels to the detector and interferes with the primary wave. At the detector, then, we have, according to Figure 1.1

$$
\begin{gathered}
\Psi_{P}=A \frac{e^{i k R_{P}}}{k R_{P}} \\
\Psi_{S}(r)=\left[A|f| \frac{e^{i k R_{j}}}{k R_{j}} e^{i \phi_{j}}\right] \frac{e^{i k R_{S}}}{k R_{S}}
\end{gathered}
$$

The total wavefunction $\Psi_{T}$, then, is

$$
\Psi_{\mathrm{T}}=\Psi_{\mathrm{P}}+\Psi_{\mathrm{S}}
$$

The total intensity $\mathrm{I}_{\mathrm{T}}$ is

$$
I_{T}=\left|\Psi_{T}\right|^{2}=\Psi_{T}^{*} \Psi_{T}=\Psi_{P}^{*} \Psi_{P}+\Psi_{P}^{*} \Psi_{S}+\Psi_{P} \Psi_{S}^{*}+\Psi_{S} \Psi_{S}^{*}
$$

Neglecting the scattered-scattered term, this becomes

$$
\begin{aligned}
& \left.I_{T}=\frac{|A|^{2}}{\left(k R_{P}\right)^{2}}+\left[A^{*} \frac{e^{-i k R_{P}}}{k R_{P}}\right]\left[A|f| \frac{e^{i k R_{j}}}{k R_{j}} e^{i \phi_{j}}\right] \frac{e^{i k R_{S}}}{k R_{S}}\right]+c . c . \\
& I_{T}=\frac{|A|^{2}}{\left(k R_{P}\right)^{2}}+\frac{2|A|^{2}|f|}{k^{3} R_{P} R_{j} R_{S}} \cos \left(k\left(R_{j}+R_{S}-R_{P}\right)+\phi_{j}\right)
\end{aligned}
$$

Noting that

$$
R_{S}=R_{P}-R_{j} \cos \theta_{j}
$$

and that $R_{P} \sim R_{S}$, we have

$$
I_{T}=\frac{|A|^{2}}{\left(k R_{P}\right)^{2}}+\frac{2|A|^{2}|f|}{k^{3} R_{P}^{2} R_{j}} \cos \left(k R_{j}\left(1-\cos \left(\phi_{j}\right)+0_{j}\right)\right.
$$

We now extract the fractional variation in $\mathrm{I}_{\mathrm{T}}$

$$
\chi(k)=\frac{I_{T}}{I_{0}}-1=\frac{\Psi_{P}^{*} \Psi_{S}+\Psi_{P} \Psi_{S}^{*}}{\Psi_{P}^{*} \Psi_{P}}-1=\frac{2|f|}{k R_{j}} \cos \left(k R_{j}\left(1-\cos \left(\theta_{j}\right)\right)+\phi_{j}\right)
$$


where $I_{0}$ is the photocurrent that would be measured in the absence of other scattering atoms. Thus, the fractional oscillation in the measured photocurrent is directly relatable to the scattering geometry and the scattering phase of the substrate atom.

There are several variants of photoelectron diffraction as a structural tool. Laboratory based methods include Azimuthal Photoelectron Spectroscopy, in which the analyzer and photon source are kept fixed in space, but where the sample is rotated to scan a longitudinal slice of the emitted electron distribution and Polar Photoelectron Spectrosccpy, where a latitudinal slice is taken. The variant of photoelectron diffraction used in this thesis is ARPEFS (Angle-Resolved Photoelectron Extended Fine Structure). In this method the sample, analyzer, and photon source are kept fixed in space and the photon energy is varied. Generally, the photocurrent is measured at several experimental geometries to gain information about the surface structure. To get a variable energy photon source with sufficient intensity to perform an experiment requires working at synchrotrons.

Let us return to Equation 1.12 and bring it further into our context of surface experiments. Repeating, ARPEFS is the mudulation of measured photoelectron intensity, measured over a $50 \mathrm{eV}-550 \mathrm{eV}$ range. The total intensity $I_{\mathrm{l}}(E)$ may be written as $I_{l}(E)=[1+\chi(E)] I_{0}(E)$, where $I_{0}(E)$ is the slowly varying (and decreasing) atomic like contribution and $\chi(E)$ is a rapidly oscillating part, caused by interference between the primary outgoing photoelectron wave and the same wave scattered from nearby atoms. In the single scattering limit, ARPEFS may be written as

$$
\chi(k)=2 \sum_{j} \frac{\cos \beta_{j}}{\cos \gamma} \frac{\left|\mathrm{f}\left(\theta_{\mathrm{j}}, k\right)\right|}{\mathrm{R}_{\mathrm{j}}} \cos \left(k \Delta \mathrm{R}_{\mathrm{j}}+\phi_{\mathrm{j}}\right) \mathrm{e}^{-\left(\sigma_{\mathrm{j}}^{2}\left(1-\cos \theta_{\mathrm{j}}\right) \mathrm{k}^{2}+\frac{\Delta \mathrm{R}_{\mathrm{i}}}{\lambda}\right)}
$$

The summation is over all atoms $\mathrm{j}$ near the adsorbed source atom. The angle $\beta_{\mathrm{j}}$ is the angle between the photon polarization vector and the vector connecting 
the emitter and scattering atoms. $\gamma$ is the angle between the photon polarization vector and the electron emission direction. $\mathbf{R}_{\mathbf{j}}$ is the bond length between the emitter and the scatterer atoms. $f\left(\theta_{j}\right)$ and $\phi\left(\theta_{j}\right)$ are the scattering amplitude and phase, respectively, of atom $\mathbf{j}$. The emission path length difference (PLD) between the direct wave and the scattered wave is given by $\Delta R_{j}=R_{j}-R_{j} \cos \theta_{j}$. Inelastic damping due to thermal averaging is included using a Debye-Waller term, where $\sigma_{j}^{2}$ is the mean-square relative displacement between the emitting and scattering atoms. Inelastic loss of photoelectron wave amplitude due to plasmon excitations and electron-hole pair creation by the energetic photoelectron are incorporated into an electron mean-free path $\lambda$. The angles $\beta, \gamma$, and hence $\theta_{\mathrm{j}}$ can be (and usually are) chosen to highlight particular backscattering atoms.

We see that ARPEFS in this form is the sum of many sinusoids of different frequencies. Such a signal can resolved into its constituents using Fourier filtering ${ }^{1}$. The Fourier transform is frequently used to reject some high symmetry adsorption sites in favor of other sites. The Fourier filtered curve may be modeled to find a structure consistent with the measured $\chi(\mathrm{E})$ curves. Another approach is to just model the $\chi(k)$ curve based on an assumed structure and maximize the agreement between the data and the theory curve. This method has been applied to a number of structures ${ }^{2-10}$

In Figure 1.2 we show a flowchart for a typical ARPEFS experiment. The first step is to decide what experimental geometries will be used for experiments at the synchrotron. If the structure is approximately known, theoretical curves are calculated to find geometries most sensitive to the desired structural parameters. If the structure is not known, $\chi(k)$ curves will be calculated for assumed high symmetry sites and reasonable estimates of the bond lengths. Geometries are then selected that will distinguish between the various putative sites and provide useful 
structural information.

In practice, this means that $\mathrm{I}(\mathrm{E})$ curves are taken at normal emission and at a few off-normal angles. Because the scattering amplitude $f$ in Equation 1.3 is strongly peaked in the forward and backscattering directions, each $\chi(\mathrm{k})$ curve is dominated by contributions from only a few atoms. The normal emission curve is dominated by atoms lying underneath the photoemitter; the off-normal curves are dominated by atoms lying behind (but not necessarily under) the photoemitter. Examples are given in Chapter 4, Figures 4.9 and 4.10, the discussion of the strengths and weaknesses of ARPEFS. In essence, then, one determines a structure by working out, a few atoms at a time, where the substrate atoms are with respect to the photoemitter.

Once one knows which experimental geometries are to be used, one goes to a synchrotron and measures the desired I(E) curves. Experimental difficulties and the steps taken to mitigate them are treated in more detail in Section 4.2. After the I(E) curves have been taken, $\chi(k)$ curves and their Fourier transforms are made.

These curves are then compared to theoretical curves. The inputs to the theory curves are varied until the agreement is deemed satisfactory (see Chapter 2, $\mathrm{p}(2 \times 2) \mathrm{S} / \mathrm{Cu}(001)$, and Chapter $3, \mathrm{p}(2 \times 2) \mathrm{S} / \mathrm{i}$ ivicili1) $)$. in cases where the adsurption site is unknown, the Fourier transforms are usually sufficient to exclude possible sites (see Chapter $3,(\sqrt{3} \times \sqrt{3}) \mathrm{R} 30^{\circ} \mathrm{S} / \mathrm{Ni}(111)$ ).

This thesis is arranged as follows: Chapter 2 contains results of ARPEFS studies on $\mathrm{p}(2 \times 2) \mathrm{S} / \mathrm{Cu}(001)$. It also contains a discussion of changes made to the ARPEFS technique for this thesis to enhance the reliability of the method. These methods are then applied to $\mathrm{p}(2 \times 2) \mathrm{S} / \mathrm{Ni}(111)$ and $(\sqrt{3} \times \sqrt{3}) \mathrm{R} 30^{\circ} \mathrm{S} / \mathrm{Ni}(111)$ in Chapter 3. Strengths and weaknesses of ARPEFS, as illustrated by the results of $\mathrm{p}(2 \times 2) \mathrm{S} / \mathrm{Cu}(001), \mathrm{p}(2 \times 2) \mathrm{S} / \mathrm{Ni}(111)$, and $(\sqrt{3} \times \sqrt{3}) \mathrm{R} 30^{\circ} \mathrm{S} / \mathrm{Ni}(111)$ are dis- 
cussed in Chapter 4. In Chapter 5 we treat an unrelated ARPES experiment on $\mathrm{Au}(001)$, in which the effects of the crystal surface on valence band photoemission are presented. 


\subsection{Figure Captions}

Figure 1.1: Schematic of interference between the primary photoelectron wave (shown with long dashes) and the scattered photoelectron wave (shown as short dashes). $R_{P}$ is the distance between the photcemitter and the analyzer, $R_{S}$ is the distance between the scatterer and the analyzer, and $R_{j}$ is the bond distance between the photoemitter and the scatterer. The angle between the interatomic bond and the photoemission direction is $\phi_{\mathrm{j}}$.

Figure 1.2: A flowchart of the steps in an ARPEFS experiment. 
Figure 1.1:

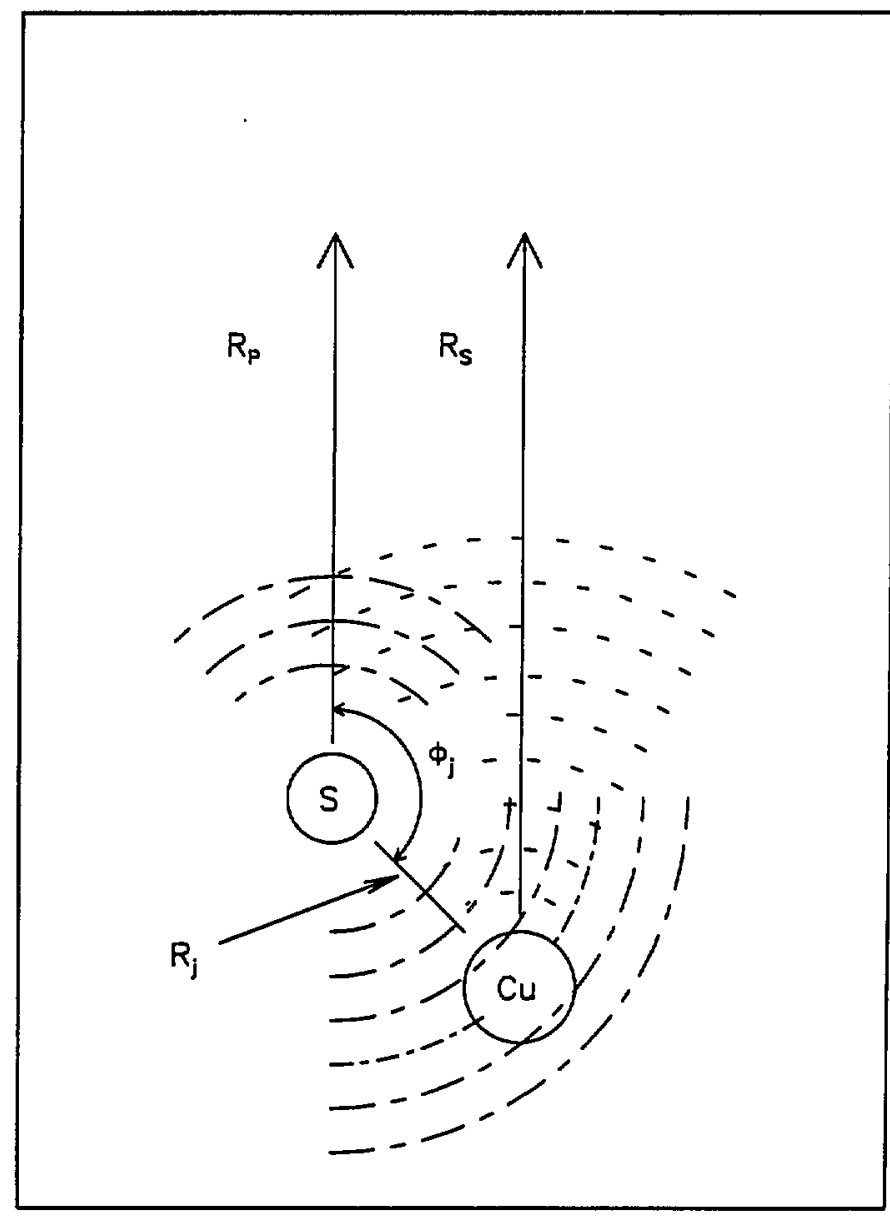


Figure 1.2:

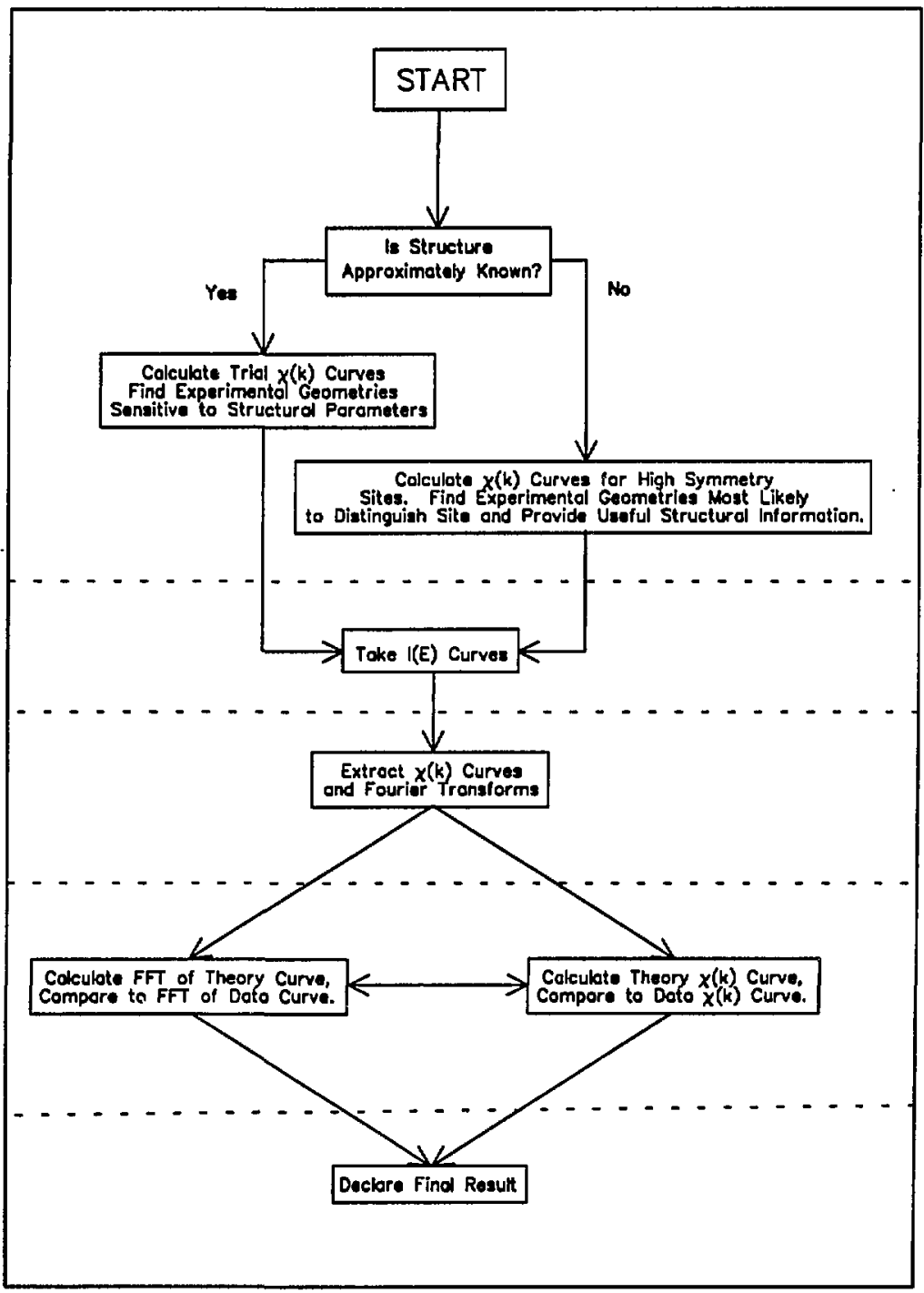




\section{Chapter 2}

\section{$\mathrm{p}(2 \times 2) \mathrm{S} / \mathrm{Cu}(001)$ ARPEFS}

\subsection{Abstract}

We have performed low temperature (110 K - $160 \mathrm{~K})$ Angle Resolved Photoemission Extended Fine Structure studies of $\mathrm{p}(2 \times 2, \mathrm{~S} / \mathrm{Cu}(001)$. Analysis of these low-temperature data using Multiple Scattering Spherical Wave calculations indi-

cates that $\mathrm{S}$ adsorbs into a fourfold hollow site $1.31 \AA$ above the $\mathrm{Cu}$ surface, with near-surface reconstruction of the $\mathrm{Cu}$ layers similar to recent LEED and Medium Energy lon Scattering results. The S-Cu bond length is determined to be $2.26 \AA$. The second-layer $\mathrm{Cu}(001)$ plane appears to be corrugated.

\subsection{Introduction}

The local atomic structure of $\mathrm{p}(2 \times 2) \mathrm{S} / \mathrm{Cu}(001)$ has been studied by a variety of techniques, including Angle Resolved Photoemission Extended Fine Structure (ARPEFS) ${ }^{2}$, Surface Extended X-ray Absorption Fine Structure (SEXAFS)/XRay Standing Wave ${ }^{11}$, Low Energy Electron Diffraction (LEED) ${ }^{12-14}$, X-Ray Diffraction (XRD) ${ }^{15}$, Electron Energy Loss Spectroscopy (EELS) ${ }^{16}$, and Medium Energy lon Scattering (MEIS) ${ }^{17}$. All of these studies agree that $S$ occupies a fourfold hollow site. They differ, however, in the quantitative details of the adsorption 
geometry and on the effect of $\mathrm{S}$ adsorption upon the local structure of the $\mathrm{Cu}$ substrate. $D_{S_{1}}$, the vertical distance between the $S$ atoms and the first $\mathrm{Cu}$ layer, has been found to be either $\sim 1.3 \AA^{14,16,17}$ or $\sim 1.4 \AA^{2,11}$. The Cu-S bond length is reported as being between $2.23 \AA$ and $231 \AA$.

Reconstruction of the near-surface layers has also been found. Under $\mathrm{p}(2 \times 2)$ symmetry, $\mathrm{Cu}$ atoms in the first layer, and all odd numbered layers, may shift parallel to the surface (see Figures 2.1a and b). There are three inequivalent sites in the even-number layers, these are the 'covered', 'open', and 'anti-covered' sites. The 'covered' $\mathrm{Cu}$ atoms are directly below $\mathrm{S}$ adatoms. In the square pattern formed by the four $\mathrm{S}$ adatoms, the 'open' sites lie under the sides of this square. The 'anti-covered' sites are in the center of the square (see Figure 2.1b). Atoms in these inequivalent sites are allowed by symmetry to shift toward or away from the surface, but not laterally. The ARPEFS ${ }^{2}$, LEED $^{14}$, and MEIS ${ }^{17}$ results were analyzed for reconstruction in the two outermost $\mathrm{Cu}$ layers, subject to the above constraints. All three found a lateral shift in the first layer Cu atoms: LEED ${ }^{14}$ and MEIS $^{17}$ found an outward shift, away from the S adatom, of $0.02 \AA$ and $0.03 \AA$, respectively, whereas $\mathrm{ARPEFS}^{2}$ found an inward shift of $0.05 \AA$. The conclusions about the second layer were as striking: ARPEFS ${ }^{2}$ found a buckling of $0.12 \AA$, LEED $^{14}$ found a buckling of $0.03 \AA$ in the opposite sense, and MEIS ${ }^{17}$ found no buckling of the second layer. A summary of published $\mathrm{p}(2 \times 2) \mathrm{S} / \mathrm{Cu}(001)$ structures and our notation is presented in Table 2.1 and Figure 2.1.

We performed new ARPEFS measurements in an effort to resolve differences between the previous ARPEFS ${ }^{2}$ result and the recent LEED ${ }^{14}$ study. Because of the small differences between the derived parameters in these two sets of results, ( $<0.1 \AA$ for the nearest neighbor $\mathrm{Cu}$ atoms, $<0.05 \AA$ for more distant $\mathrm{Cu}$ atoms), high accuracy was essential. In this work we have therefore made a significant 
departure from earlier ARPEFS methodology.

We have sought to increase the magnitude of the ARPEFS oscillations by performing the measurements at a low sample temperature $(110 \mathrm{~K}-160 \mathrm{~K})$. Model MSSW calculations indicate that the structural differences between the published ARPEFS $^{2}$ and LEED ${ }^{14}$ models affect $\chi(k)$ curves primarily at higher wavevector values. It is at these larger values of $k$, however, that photoelectron diffraction is most strongly attenuated by thermal motion of the subsirate atoms (see Eq. 1.13). Experimental ${ }^{18,19}$ and theoretical ${ }^{20}$ studies of bulk $\mathrm{Cu}$ indicate that cooling $\mathrm{Cu}$ to $\sim 150$ K would reduce $\sigma_{j}^{2}$ for first shell atoms by at least $40 \%$. Model cialculations indicated that while at room temperature the two structures would yield qualitatively similar $\chi(\mathrm{k})$ curves for most of the trial experimental geometries investigated, cooling the $\mathrm{Cu}$ substrate to below $\sim 150 \mathrm{~K}$ would increase the magnitude of the ARPEFS oscillations sufficiently to resolve differences between them.

\subsection{Experimental}

A mechanically polished and chemically etched $\mathrm{Cu}(001)$ crystal was attached with tantalum strips to a tantalum sample plate, whicti was in turn mounted on a threeaxis manipulator equipped with $\mathrm{LN}_{2}$ cooling coils. The manipulator was installed in a UHV chamber equipped with an ion-sputtering gun, four-grid LEED/Auger optics and a moveable hemispherical analyzer ${ }^{21,22}$. The crystal was cleaned by repeated cycles of $\mathrm{Ar}^{+}$sputtering $\left(1 \mathrm{kV}, 10^{-5}\right.$ torr $\left.\mathrm{Ar}^{+}\right)$and annealing to $750 \mathrm{~K}$ until sharp $(1 \times 1)$ LEED patterns were obtained and $S, C$, and $O$ were not detectable by Auger spectroscopy. Ambient dosing of the clean room-temperature crystal with $60 \mathrm{~L}$ ( $120 \mathrm{sec} .5 \times 10^{-7}$ torr) $\mathrm{H}_{2} \mathrm{~S}$, followed by annealing to $550 \mathrm{~K}$ produced a sharp stable $\mathrm{p}(2 \times 2)$ overlayer. Temperatures were measured using a chromel-alumel thermocouple (referenced to room-temperature) attached to the sample plate near 
the crystal.

Sulfur 1s ARPEFS data were taken using the double crystal monochromaiors on Beamline $3-3^{23}$ at the Stanford Synchrotron Radiation Laboratory (SSRL, and on Beamline X-24A at the National Synchrotron Light Source ${ }^{24}$. Photoemission spectra were taken in the $50 \mathrm{eV}-550 \mathrm{eV}\left(\mathrm{k}=4.4 \AA^{-1}\right.$ to $\left.12 \AA^{-1}\right)$ kinetic energy range using 2525 ? $3025 \mathrm{cV}$ photons. The analyzer was of $\cdots \cdot d$ at $160 \mathrm{eV}$ pass energy, giving an estinated overall energy resolution of $\sim 2 \mathrm{sV}$. The charaber base pressure was $6 \times 10^{-11}$ torr to $3 \times 10^{-10}$ torr. During data collection the crystal was flashed periodically to $550 \mathrm{~K}$ to desorb any adsorbed contaminants.

Sulfur ls photoemiission spectra were taken in $0.07 \AA^{-1}$ to $0.1 \AA^{-1}$ increments over the energy range specified above. A total of 90 to 100 such spectra thus constitute a complete $\chi(\mathrm{k})$ curve. Two normal emission $\chi(\mathrm{E})$ curves were obtained, one at a sample temperature of $\mathrm{T}=110 \mathrm{~K}$ and one at a sample temperature of $\mathrm{T}=155 \mathrm{~K}$. There are also two off-normal emission curves, one at $40^{\circ}$ off normal $(\mathrm{T}=140 \mathrm{~K})$ in the $[011]$ direccion and one at $50^{\prime \prime}$ off normal $(\mathrm{T}=110 \mathrm{~K})$ in the [011] direction, for a total of four curves.

\subsection{Discussion}

The ARPEFS data were reducet in the standard fashion. Each individual $S$ is photoemission spectrum was fitted as a sum of an empirical background iunction, a Voigt function, and a Gaussian broadened step function. The Lorentzian portion of the Voigt function was fixed at $0.8 \mathrm{eV}$. The means and Gaussian widths of the Voigt function and of the step function were constrained to be equal. Thus, each individual photoemission spectrum was described by five parameters: the area of the Voigt function, the height of the step fus.ztion, the mean and Gaussian width of the Voigt function, and the scale factor applied tc the empirical background 
function. We then constructed an $\mathrm{I}(\mathrm{E})$ curve by plotting the area of the Voigt function as a function of the Voigt mean, using the scaling factor of the empirical background to normalize each spectrum. In an EXAFS-like manner we fitted a quadratic or cubic polynomial $I_{0}$ to these raw $\mathrm{I}(\mathrm{E})$ curves and formed $\chi(E)$ curves using $\mathrm{I}_{t}(\mathrm{E})=[1+\chi(E)] \mathrm{I}_{0}(\mathrm{E})$. The resulting $\chi(E)$ curves are shown in Figure 2.2.

We calculated theoretical $\chi(k)$ curves using the MSSW method described elsewhere ${ }^{25}$. Briefly, this model for ARPEFS calculates the interference between the primary photoelectron wave and the photoelectron waves scattered by atoms in the substrate. The method uses spherical waves and models the thermal vibration of the adsorbate and substrate atoms using a correlated Debye model. Effects of the finite angular acceptance of the analyzer and inelastic mean free path are also included.

There are three basic classes of parameters in any MSSW calculation. In order of decreasing importance they are the structural, experimental, and non-structural parameters. In fitting our data we assume the same structural constraints used elsewhere ${ }^{2,14,17}$. Given the $p(2 \times 2)$ overlayer and assumed $C_{4 v}$ local symmetry the atoms in the first $\mathrm{Cu}$ layer are constrained to be coplanar and are allowed to shift radially with respect to the $S$ adsorbate. The atoms in second layer are allowed to shift vertically, but not horizontally. For simplicity and consistency with the earlier works ${ }^{2,14,17}$ we assume the atoms in the third and subsequent layers to be in their bulk positions. We thus have six structural parameters: $D_{S 1}$ (the vertical distance between the $\mathrm{S}$ adatom and the first $\mathrm{Cu}$ layer), $\mathrm{D}_{\mathrm{SC}}$ (the vertical disiance between the $\mathrm{S}$ adatom and the 'covered' $\mathrm{Cu}$ atom in the second layer), $\mathrm{D}_{\mathrm{SO}}$ (the vertical distance between the $\mathrm{S}$ adatom and the 'open' $\mathrm{Cu}$ atom in the second layer), $\mathrm{D}_{\mathrm{SA}}$ (the vertical distance between the $\mathrm{S}$ adatom and the 'anti-covered' $\mathrm{Cu}$ atom in the second layer), $D_{S 3}$ (the vertical distance between the $S$ adatom and 
the third $\mathrm{Cu}$ layer), and the S-Cu bond length (see Figure 2.1). Note that any horizontal displacement of atoms in the first $\mathrm{Cu}$ layer is implicitly defined by $\mathrm{D}_{\mathrm{S1}}$ and the bond length.

There are five experimental parameters as well: the photoemission direction $\left(\hat{\theta}_{e}, \hat{\phi}_{\mathrm{e}}\right)$, the photon polarization $\left(\hat{\theta}_{\mathrm{h} \nu}, \hat{\phi}_{\mathrm{h} \nu}\right)$, and the temperature of the sample during the ARPEFS experiment. In these experiments $\hat{\phi}_{h \nu}$ was always equal to $\hat{\phi}_{e}$ and in the [011] plane. For the off-normal measurements $\hat{\phi}_{h \nu}$ and $\hat{\theta}_{e}$ were also equal. In the normal emission experiments the difference $\hat{\theta}_{h \nu}-\hat{\theta}_{e}$ was set to $40^{\circ}$. Because of the experimental difficulties in determining $\hat{o}_{e}$ and $\hat{\phi}_{e}\left(\right.$ see Barton ${ }^{1}$ ) precisely, these angles were treated as adjustable parameters in the fitting.

Finally there are such non-structural parameters as the Debye temperatures of the adsorbate and substrate, angular acceptance of the analyzer, the value of the inelastic mean free path $\lambda$, and the value of $V_{0}$ used in the deBroglie relation when converting from kinetic energy to wavevector. The bulk Debye temperature ${ }^{26}$ for $\mathrm{Cu}$ was taken to be $320 \mathrm{~K}$. The surface Debye ${ }^{27}$ temperature for $\mathrm{Cu}$ was initially set at $184 \mathrm{~K}$. The inelastic mean free path ${ }^{28}$ was set at $\lambda=0.75 \mathrm{k}$, where $\lambda$ is in units of $\AA$ and $k$ is in units of $\AA^{-1}$. The angular acceptance of the analyzer is taken to be $3^{\circ}$.

Phase shifts were calculated on a superposition potential ${ }^{29}$ using modified programs by Loucks ${ }^{30}$, using free alom wavefunctions ${ }^{31}$. The phaseshifts for sulfur were based on a hypothetical bcc lattice with lattice constant $2.26 \AA$. The lattice constant for $\mathrm{Cu}$ was taken to be $3.606 \AA$. This is the average of the $160 \mathrm{~K}$ and $110 \mathrm{~K}$ values ${ }^{32,33}$. The exchange potential was modelled using the Slater $\mathrm{X} \alpha$ approximation, with $^{34} \alpha=0.77$.

To determine the values in Table 2.2, we thus fit each $\chi(E)$ curve with ten adjustable parameters (the six structural parameters, the two angles needed to 
define the experimental geometry (i.e. $\hat{\theta}_{e}, \hat{\phi}_{e}$ ), and the isotropic adsorbate and substrate surface Debye temperatures).

\subsection{Parameter Determination}

Equation 1.13 may be rewritten as

$$
\chi(\mathrm{k})=\sum_{\mathrm{j}} \mathrm{A}\left(\beta, \gamma, \mathrm{k}, \theta_{\mathrm{j}}, \mathrm{R}_{\mathrm{j}}\right) \cos \left(\mathrm{k} \Delta \mathrm{R}_{\mathrm{j}}+\phi\left(\theta_{\mathrm{j}}\right)\right),
$$

where $A\left(\beta, \gamma, k, \theta_{j}, R_{j}\right)$ is an envelope function. As mentioned in Section 1.1, the angles $\beta, \gamma$, and $\theta_{\mathrm{j}}$ are usually chosen to maximize scattering from a particular atom or set of atoms. ARPEFS in the form of Eq. 2.1 is similar to EXAFS and much ARPEFS analysis has been performed using techniques that are standard in EXAFS $^{35}$. The $\chi(k)$ curve may be Fourier transformed to obtain path length differences and to yield bonding geometries. Additionally, peaks in the Fourier transform may be back transformed to extract approximate structural information by comparison with a single-scattering model. While this method works best for offnormal ARPEFS measurements, it is inappiopriate for normal emission spectra ${ }^{3}$. Since we are interested in high accuracy and because the $\mathrm{p}(2 \times 2) \mathrm{S} / \mathrm{Cu}(001)$ structure is already approximately known, this particular approach will not be pursued further. Instead we will concentrate solely on a MSSW analysis.

We minimize the R-factor (see Section 2.8)

$$
R_{N}=\frac{1}{N} \sum_{k_{i}}\left[\chi_{T}\left(k_{i}\right)-\chi_{E}\left(k_{i}\right)\right]^{2}
$$

Here, $\chi_{\mathrm{T}}\left(\mathrm{k}_{\mathrm{i}}\right)$ is theoretical value of $\chi\left(\mathrm{k}_{\mathrm{i}}\right)$ calculated using our MSSW model and $\chi_{E}\left(k_{i}\right)$ is the experimental value of $\chi\left(k_{i}\right) . N$ is number of data points. We used a modified $^{36}$ version of the simplex algorithm of Nelder and Mead ${ }^{37,38}$ to minimize Eq. 2.2. Once 'convergence' had been achieved, a plot of $R$-factor versus parameter 
was made to determine error bars. Results of the process are shown in Table 2.2, along with the best $\mathrm{R}$-factor.

Following reference 39 , we performed the same analysis on the unweighted Fourier transforms of the $\chi(k)$ curves, that is, fitting the Fourier transforms of the $\chi(\mathrm{k})$ curves with the Fourier transforms of theoretical $\chi(\mathrm{k})$ curves. Since we would expect this to give the same result as fitting in $k$-space, this gives us an idea of the uncèrtainty of the fitting process. Results of this fitting process are given in Table 2.3. The fits are shown in Figures $2.3-2.6$.

\subsection{Analysis}

\subsubsection{Normal Emission, $110 \mathrm{~K}$ and $155 \mathrm{~K}$}

The results of fitting these $\chi(E)$ curves in both $\mathrm{k}$ - and $\mathrm{F}$-spaces are shown in Figure 2.3 - 2.4. R-space and $\mathrm{k}$-space fits generally converged to the same structure (see Tables 2.2 and 2.3); the sole exception being the value for $D_{S 1}$ obtained by fitting the $110 \mathrm{Ir}$ data in $\mathrm{R}$-space. In the $155 \mathrm{~K}$ data we note that the amplitude of the oscillations in $\chi(\mathrm{E})$ below $150 \mathrm{eV}$ are underestimated by the theoretical curve, this problem is less severe for the $110 \mathrm{~K}$ curve. The regions around $200 \mathrm{eV}$ and $330 \mathrm{eV}$ are poorly described; the data show a smooth variation in $\chi(E)$ here at both temperatures whereas the theory curves all show double-peaked structures here. We note that for both the $110 \mathrm{~K}$ data and the $155 \mathrm{~K}$ data there is good agreement in peak positions between the data and theory Fourier transf ms. Peak amplitudes are better matched for the $110 \mathrm{~K} \mathrm{data}$, though we suspect most of the low-frequency discrepancy for the $155 \mathrm{~K}$ curves is due to truncation effects on the low-energy side of the data. Finally we note the overall increase seen in the Fourier amplitudes for the $110 \mathrm{~K}$ data compared with the $155 \mathrm{~K}$ data (note the difference in scale between Figures 2.3b,d versus Figures 2.4b,d. 


\subsection{2 $40^{\circ}$ Off Normal Emission, $140 \mathrm{~K}$}

We note that, as with the normal emission data, that we are unable to fit the data below approximately $100 \mathrm{eV}$. In this emission direction (Figure 2.5) we are unable to match the rapid oscillation in this area. We are also unable to match the relative heights in the shoulders seen as the $\chi(\mathrm{E})$ curve goes through the minimum at $\sim 170 \mathrm{eV}$. We see that there is good agreement in the peak positions in the Fourier transforms for both the k-space and R-space fits. We see in Figure 2.5d that improvement in agreement in the Fourier amplitudes below $\sim 9 \AA$ comes at the expense of peak position matchup for the longer path length differences.

\subsection{3 $50^{\circ}$ Off Normal Emission. $110 \mathrm{~K}$}

The fitting of these data shows many of the same difficulties seen for the other three $\chi(\mathrm{E})$ curves. The amplitude of the $\chi(\mathrm{E})$ curve is poorly modelled below $\sim 90 \mathrm{eV}$. The fitting in $\mathrm{k}$-space otherwise yields good visual agreement in $\mathrm{k}$-space an also gives good matchup in the peak positions in the Fourier transforms. Fitting in $R$-space improves the agreement for the dominant peak, but, as was found with the $40^{\circ}$ off-normal emission data, this is at the expense of the other peaks.

\subsubsection{Overview}

In Figures 2.7 - 2.12 we show graphs of $R$-factor as each structural parameter is varied. For $D_{\$ 1}$ we note that the k-space fits have minima between $1.31 \AA$ and $1.37 \AA$. The R-space fits show poor agreement for this parameter, scattering widely about the mean value $1.30 \AA$. The difference seen in the results for the 'best' value of $D_{51}$ for the $110 \mathrm{~K}$ normal emission curve appears related to our inability to fit the features at $200 \mathrm{eV}$ and $300 \mathrm{eV}$ kinetic energy: the larger value for $D_{S_{1}}$ gives better agreement at $300 \mathrm{eV}$ at the expense of the agreement at $200 \mathrm{eV}$; the problem 
is opposite for the smaller value of $D_{S 1}$. The agreement in the Fourier transforms (Figures 2.3b and 2.3d) appears equally fair for both values of $D_{S_{1}}$. The normal emission curves give well defined and consistent results for the parameter $D_{\mathrm{SC}}$. This is consistent with ARPEFS' being sensitive to atoms lying along the electron emission direction. The photoemission paths to atoms ' $O$ ' and ' $A$ ' in the second layer are getting out of alignment with the primary photoemission path to the detector; this and ine increased pathlength differences yield less certainty in the determinations of $D_{S O}$ and $D_{S A}$. The results for $D_{S 3}$ are similar to $D_{S C}$; the normal emission curves give well defined values for the $\mathrm{S}$ to third layer $\mathrm{Cu}$ distance.

The off normal emission curves complement the normal emission results. The former give more precise values for the S-Cu bond length, again because ARPEFS is sensitive to atoms lying along the photoemission direction. Both off-normal emission curves have large uncertainty in the position of atom ' $\mathrm{C}$ '; this is because the atom ' $\mathrm{C}$ ' would have to scatter the photoelectron wave at an angle of $130^{\circ}$ to $140^{\circ}$ to appear in the off-normal emission curves. These angles correspond to an angular minimum in the scattering strength of $\mathrm{Cu}$, hence the poor definition of $\mathrm{D}_{\text {SC }}$ by the off normal curves.

The $50^{\circ}$ off normal emission curve is dominated by backscattering from the first layer. While this gives us a good estimate of the S-Cu bond length, it does not give us a reliable estimate for $D_{\mathrm{S1}}$. Analysis of the path length differences for scattering from the four $\mathrm{Cu}$ atoms nearest the $\mathrm{S}$ adatom indicates that this information would be contained as a weak shoulder on the low- $R$ side of the main peak in the Fourier transform, and would be obscured by the main peak.

By fitting parabolas to the bottoms and sides of the curves in Figures 2.7-2.12 we extracted the parameters and their uncertainties given in Tables 2.2 and 2.3. Because of the scatter in the parameter determinations and because in many cases 
the minima of the curves in Figures 2.7-2.12 are poorly described by parabolas we used these uncertainties as relative weights only (see Section 2.8) in determining the values given in Table 2.4 .

\subsection{Conclusion}

There is appears to be general agreement now about the first layer reconstruction of $\mathrm{p}(2 \times 2) \mathrm{S} / \mathrm{Cu}(001)$. ARPEFS, LEED, and MEIS all indicate that the $S$ atom is $1.29 \AA$ to $1.32 \AA$ above the surface (with ARPEFS giving the largest value, 1.315(13) $\AA$, for this parameter). Our value of $2.26 \AA$ for the S-Cu bond length implies a $0.03 \AA$ outwards relaxation of the first layer $\mathrm{Cu}$ atoms, in agreement with LEED $^{14}$, MEIS ${ }^{17}$, and XRD ${ }^{15}$. There is remains a strong quantitative difference regarding the second layer $\mathrm{Cu}$ atoms. Qualitatively, all three methods agree that if there is a reconstruction of the second layer, it is in the direction $D_{\mathrm{SC}}>\mathrm{D}_{\mathrm{SO}}>\mathrm{D}_{\mathrm{SA}}$. Quantitatively, however, ARPEFS indicates that the buckling is $>0.08 \AA$, LEED yields $0.03 \AA$, and MEIS concludes that the buckling is $0.00 \AA$. That this large reconstruction of the second layer was found in both the R- and k-space fits indicates that ARPEFS at the present level of theory indeed yields this large a value.

\subsection{Error Analysis}

The R-factor of Eq. 2.2 is chosen for its similarity to the standard $\chi^{2}$ statistic

$$
\chi_{\nu}^{2}=\frac{1}{\nu} \sum_{\mathbf{k}_{\mathbf{i}}} \frac{\left[\chi_{\mathbf{T}}\left(k_{\mathrm{i}}\right)-\chi_{\mathrm{E}}\left(\mathrm{k}_{\mathrm{i}}\right)\right]^{2}}{\sigma_{\mathbf{k}_{\mathbf{i}}}^{2}}
$$

The definitions of the terms are the same as in Eq. 2.2, with the addition of $\nu$, the number of degrees of freedom $(\nu=\mathrm{N}-1$, where $\mathrm{N}$ is the the number of statistically independent points), and $\sigma\left(k_{i}\right)$, the noise estimate associated with 
point $k_{\mathfrak{i}}$. From considerations of the Nyquist sampling frequency we estimate, for Fourier filtered data, $N=-\Delta k \Delta R$, where $\Delta k=k_{\max }-k_{\min }$ is the range of the data and $\Delta R=R_{\max }-R_{z i n}$ is the width of the $R$-space window ${ }^{35,39}$.

To minimize Eq. 2.3, we need an estimate for $\sigma\left(\mathrm{k}_{\mathrm{i}}\right)$. We may write ${ }^{39}$ $\sigma_{\text {total }}^{2}=\sigma_{\text {random }}^{2}+\sigma_{\text {systematic }}^{2}$ We estimate our random noise level to be between $2 \%$ and $4 \%$. We have found that adding a random $3 \%$ noise to a (necessarily smooth) theoretical $\chi(k)$ curve yields a curve that visually resembles a real $\chi(k)$ curve in roughness. This is the same estimate that we get by looking at the magnitude of the high $(R>18 \AA$ ) frequency components of our $\chi(k)$ curves. We have, unfortunately, no method of estimating the magnitude of any systematic errors in either the data reduction or in the theory. Were we to assume that $\sigma_{\text {syatematic }}=0$, we would have $\sigma_{\text {total }} \sim 0.03$. Since for a 'moderately good'38 fit $\chi_{\nu}^{2} \sim 1$, this implies that our $R$-factor as defined in Eq. 2.2 should be quite close to $R_{N}=0.0009$. As is shown in Table 2.2, this is not the case. In order to use the $\chi_{\nu}^{2}$ statistic we arbitrarily set $\sigma\left(k_{i}\right)=1$ for all $k_{i}$ and proceed with the minimization, no longer having any independent evaluation of the goodness-of-fit of the result. Once the best $\chi_{\nu}^{2}$ (or $\mathrm{R}_{\mathrm{N}}$ ) has been obtained, we can estimate ${ }^{40}$ the uncertainty $\sigma_{\mathrm{P}_{j}}$ in any given parameter $P_{j}$ by

$$
\sigma_{\mathrm{P}_{\mathrm{j}}}^{2}=2 \chi_{\nu_{\text {beat }}^{2}}^{2} \times\left|\frac{\partial^{2} \chi^{2}}{\partial \mathrm{P}_{\mathrm{j}}^{2}}\right|^{-1}
$$

where $\chi^{2}=\nu \chi_{\nu}^{2}$ and $\chi_{\nu_{\text {bet }}}^{2}$ is the lowest value of $\chi_{\nu}^{2}$ found. The partial derivatives in Eq. 2.4 are evaluated by fitting a parabola to the minimum of a graph of $R_{N} v s$. $P_{j}$.

Once we have determined $P_{j}$ and $\sigma_{j}$, we estimate $\bar{P}$, the average value of $P$, and $\bar{\sigma}$, the uncertainty of $\bar{P}$, using

$$
\frac{1}{\bar{\sigma}^{2}}=\sum_{j} \frac{1}{\sigma_{j}^{2}}
$$


and

$$
\bar{P}=\bar{\sigma}^{2} \sum_{j} \frac{P_{j}}{\sigma_{j}^{2}}
$$

Because of the scatter in the individual parameter determinations, we have chosen to regard the individual $\sigma_{i}$ as relative weights instead of absolute weights. While this does not affect the determination of $\bar{P}$, the uncertainty becomes

$$
\bar{\sigma}^{2}=\frac{\sigma^{2}}{\mathrm{~N}-1} \sum_{\mathrm{j}} \frac{1}{\sigma_{\mathrm{j}}^{2}}\left(\mathrm{P}_{\mathrm{j}}-\overline{\mathrm{P}}\right)^{2}
$$

The uncertainties in Table 2.4 are given by the larger of the values determined using Eqs. 2.5 or 2.7 .

\subsection{Comparison with Previous Work}

We note that the ARPEFS oscillations are enhanced by going to a low experimental temperature. In Fig. 2.13 we show the normal emission curve from reference 2, taken at $300 \mathrm{~K}$, and the normal emission curve, taken at $110 \mathrm{~K}$, from the present study. The increase in the magnitude of oscillation is evident. Given the internal consistency of the values in Table 2.2, we decided to fit the older ARPEFS data from reference 2. We were able to obtain a good fit for the normal emission curve. Results of the k-space only fitting are shown in Table 2.5, along with the value of the R-factor obtained using the structure given in reference 2. In Figure 2.14 we show the best fit. We note that use of the simplex algorithm instead of a hand search has resulted in a better fit and that the error bars are smaller for the normal emission curves taken at low temperature. This underscores the usefulness of the changes in the ARPEFS technique described in Section 2.2. 


\subsection{Acknowledgements}

This work was supported by the Director, Office of Energy Research, Office of Basic energy Sciences, Chemical Sciences Division of the U.S. Department of Energy under Contract No. DE-AC03-76SF00098. It was performed at the Stanford Synchrotron Radiation Laboratory, which is supported by the Department of Energy's Office of Basic Energy Sciences, and at the National Synchrotron Light Source, which is supported by the Department of Energy under Contract No. DE-AC0276CH00016. We are deeply grateful to B. Karlin and D. Lindle for their assistance at NSLS. We also thank Prof. Gustafsson for sending us the MEIS results prior to publication. 


\subsection{Tables}

Table 2.1: Summary of structural parameters. Uncertainties in the last digit of each parameter, where given, are in parentheses.

\begin{tabular}{lllllllc}
\hline $\mathrm{D}_{\mathrm{S} 1}$ & $\mathrm{D}_{\mathrm{SC}}$ & $\mathrm{D}_{\mathrm{SO}}$ & $\mathrm{D}_{\mathrm{SA}}$ & $\mathrm{D}_{\mathrm{S3}}$ & $\mathrm{D}_{\mathrm{S}-\mathrm{Cu}}$ & $\Delta$ & Method \\
\hline $1.42(2)$ & $3.04(2)$ & $3.07(6)$ & $3.16(5)$ & $4.97(6)$ & $2.26(1)$ & $-0.05(2)$ & ARPEFS $^{2}$ \\
$1.28(3)$ & 3.12 & 3.11 & 3.09 & 4.94 & $2.23(6)$ & +0.02 & LEED $^{14}$ \\
1.30 & 3.11 & 3.11 & 3.11 & -4.93 & 2.25 & +0.03 & MEIS $^{17}$ \\
$1.19(14)$ & - & - & - & - & - & $+0.03(1)$ & XSW ${ }^{15}$ \\
$1.40(4)$ & - & - & - & - & $2.31(2)$ & - & SEXAFS $^{11}$ \\
$1.30(5)$ & - & - & - & - & - & - & ELS $^{16}$ \\
\hline
\end{tabular}


Table 2.2: Results of $k$-Space Fitting

\begin{tabular}{c|ccccc}
\hline Parameter & \multicolumn{2}{|c}{ Normal Emission } & \multicolumn{3}{c}{ Off Normal Emission } \\
& $110 \mathrm{~K}$ & $155 \mathrm{~K}$ & $140 \mathrm{~K}, 40^{\circ}$ & $110 \mathrm{~K}, 50^{\circ}$ \\
\hline $\mathrm{D}_{\mathrm{S1}}$ & \multicolumn{2}{|c}{$1.309(15)$} & $1.319(12)$ & $1.341(09)$ & $1.368(29)$ \\
$\mathrm{D}_{\text {SC }}$ & $3.186(14)$ & $3.171(13)$ & $3.165(33)$ & $3.223(78)$ \\
$\mathrm{D}_{\text {So }}$ & $3.111(23)$ & $3.130(18)$ & $3.190(19)$ & $3.115(23)$ \\
$\mathrm{D}_{\mathrm{SA}}$ & $3.069(31)$ & $3.092(23)$ & $3.083(31)$ & $3.052(22)$ \\
$\mathrm{D}_{\mathrm{S3}}$ & $4.894(17)$ & $4.911(15)$ & $4.916(36)$ & $4.889(31)$ \\
Bond & $2.247(17)$ & $2.265(21)$ & $2.268(07)$ & $2.269(06)$ \\
$\Delta$ & $+0.023(17)$ & $+0.037(27)$ & $0.026(10)$ & $+0.007(32)$ \\
Vo $_{\text {o }}$ & 12.7 & 11.7 & 10.7 & 11.6 \\
R-factor & .013 & .006 & .007 & .006 \\
\hline
\end{tabular}

Table 2.3: Results of R-Space Fitting

\begin{tabular}{|c|c|c|c|c|c|}
\hline \multirow[t]{2}{*}{ Parameter } & \multicolumn{2}{|c|}{ Normal Ernission } & \multicolumn{3}{|c|}{ Off Normal Emission } \\
\hline & $110 \mathrm{~K}$ & $155 \mathrm{~K}$ & & $\mathrm{~K}, 40^{\circ}$ & $110 \mathrm{~K}, 50^{\circ}$ \\
\hline$\overline{D_{S 1}}$ & $1.371(19$ & 1.309 & & $1.312(14)$ & $1.254(11)$ \\
\hline$D_{S C}$ & $3.180(13$ & 3.191 & & $3.216(58)$ & $3.219(81)$ \\
\hline$D_{\text {so }}$ & $3.122(27$ & 3.120 & & $3.153(19)$ & $3.188(43)$ \\
\hline $\mathrm{D}_{\mathrm{SA}}$ & $3.079(36$ & 3.019 & & $3.030(22)$ & $3.019(35)$ \\
\hline$D_{S 3}$ & $4.891(18$ & 4.911 & & $4.833(22)$ & $5.101(81)$ \\
\hline Bond & $2.254(16$ & 2.257 & & $2.245(07)$ & $2.259(08)$ \\
\hline$\Delta$ & $-0.014(25$ & +0.036 & & $+0.019(13)$ & $+0.076(12)$ \\
\hline$V_{0}$ & 12.6 & 11.2 & & 12.5 & 11.6 \\
\hline
\end{tabular}

Table 2.4: Average Value of Parameter

\begin{tabular}{c|rrr}
\hline Parameter & k Space Fitting & R Space Fitting & Combined Average \\
\hline $\mathrm{D}_{\mathrm{S} 1}$ & $1.330(09)$ & $1.295(23)$ & $1.315(13)$ \\
$\mathrm{D}_{\mathrm{SC}}$ & $3.178(09)$ & $3.187(09)$ & $3.182(06)$ \\
$\mathrm{D}_{\mathrm{SO}}$ & $3.141(19)$ & $3.1 \ldots i 12)$ & $3.141(11)$ \\
$\mathrm{D}_{\mathrm{SA}}$ & $3.073(13)$ & $3.034(15)$ & $3.056(10)$ \\
$\mathrm{D}_{\mathrm{S3}}$ & $4.903(10)$ & $4.890(23)$ & $4.897(11)$ \\
Bond & $2.267(04)$ & $2.252(05)$ & $2.260(04)$ \\
$\Delta$ & $+0.033(08)$ & $+0.039(17)$ & $+0.035(11)$ \\
\hline
\end{tabular}


Table 2.5: Results of $k$-Space Fitting of Normal Emission $300 \mathrm{~K}$ Data. The 'Old $\mathrm{R}$-Factor' is based on the structure given in reference 2.

\begin{tabular}{c|c}
\hline Pa:ameter & $300 \mathrm{~K}$ Normal Emission \\
\hline $\mathrm{D}_{\mathrm{S} 1}$ & $1.313(17)$ \\
$\mathrm{D}_{\mathrm{SC}}$ & $3.181(15)$ \\
$\mathrm{D}_{\mathrm{So}}$ & $3.065(26)$ \\
$\mathrm{D}_{\mathrm{SA}}$ & $3.052(35)$ \\
$\mathrm{D}_{\mathrm{S3}}$ & $4.935(29)$ \\
Bond & $2.236(30)$ \\
$\Delta$ & $+0.002(39)$ \\
$\mathrm{V}_{\circ}$ & 12.62 \\
$\mathrm{R}$-factor & .0016 \\
Old R-factor & .0062 \\
$\mathrm{~N}$ & 26 \\
\hline
\end{tabular}




\subsection{Figure Captions}

Figure 2.1: Schematic of $\mathrm{p}(2 \times 2) \mathrm{S} / \mathrm{Cu}(001)$, showing siructure and label definitions.

a) Top view, showing outwards reconstruction of first layer $\mathrm{Cu}$ atoms.

b) Side view, showing vertical relaxations of second layer $\mathrm{Cu}$ atoms.

Figure 2.2: Raw $\chi(E)$ Curves.
(a) Normal Emission (110 K).
(b) Normal Emission (155 K).
(c) $40^{\circ}$ Off Normal Emission (140 K).
(d) $50^{\circ}$ Off Normal Emission $(110 \mathrm{~K})$.

Figure 2.3: Results of fitting the normal emission (110 K) datil in $k$-space and $\mathrm{R}$-space. The $\mathrm{k}$-space curves are Fourier filtered at $1.4 \AA \because \mathrm{R}<14.4 \AA$.

(a) and (c) Comparison of data (dots) and theory (solid lint) curves. Fit performed in k-space.

(b) and (d) Comparison of data (dots) and theory (solid line) curves. Fit performed in R-space.

Figure 2.4: Results of fitting the normal emission ( $155 \mathrm{~K})$ data in $k$-space and R-space. The k-space curves are Fourier filtered ai $1.2 \AA<\mathrm{R}<12.8 \AA$. (a) and (c) Comparison of data (dots) and theory (solid line) curves. Fit performed in $\mathrm{k}$-space.

(b) and (d) Comparison of rata (dots) and theory (solid line) curves. Fit performed in R-space. 
Figure 2.5: Results of fitting the $40^{\circ}$ off normal emission $(140 \mathrm{~K})$ data in $k$-space and R-space. The k-space curves are Fourier filtered at $2.1 \AA<\mathrm{R}<15.4 \AA$. (a) and (c) Comparison of data (dots) and theory (solid line) curves. Fit performed in k-space.

(b) and (d) Comparison of data (dots) and theory (solid line) curves. Fit performed in R-space.

Figure 2.6: Results of fitting the $50^{\circ}$ off normal emission ( $\left.110 \mathrm{~K}\right)$ data in $\mathrm{k}$-space and R-space. The k-space curves are Fourier filtered at $2.4 \AA<R<13.8 \AA$. (a) and (c) Comparison of data (dots) and theory (solid line) curves. Fit performed in k-space.

(b) and (d) Comparison of data (dots) and theory (solid line) curves. Fit performed in R-space.

Figure 2.7: R-Factor vs. $D_{S 1}$, the vertical distance between the $S$ adatom and the first $\mathrm{Cu}$ layer. The solid lines are cubic spline interpolations as guides to the eye. For ease of comparison all curves have been rescaled to have the value 1 at the minimum. The minima show large scatter within the range $\sim$ $1.3 \AA$ to $1.35 \AA$.

Symbols:

Filled Squares: Normal emission, $110 \mathrm{~K}$.

Filled Circles: Normal emission, $155 \mathrm{~K}$.

Open Circles: $40^{\circ}$ Off normal emission, $140 \mathrm{~K}$.

Open Squares: $50^{\circ}$ Off normal emission, $110 \mathrm{~K}$. 
Figure 2.8: Residuals vs. $D_{S C}$, the vertical distance between the $S$ adatom and the 'covered' $\mathrm{Cu}$ atom in the second layer. The solid lines are cubic spline interpolations as guides to the eye. For ease of comparison all curves have been rescaled to have the value 1 at the minimum. Note that the curves for the normal-emission data show well defined and reproducible minima for this parameter, whereas the off-normal data are quite insensitive to this parameter.

Symbols:

Filled Squares: Normal emission, $110 \mathrm{~K}$.

Filled Circles: Normal emission, $155 \mathrm{~K}$.

Open Circles: $40^{\circ}$ Off normal emission, $140 \mathrm{~K}$.

Open Squares: $50^{\circ}$ Off normal emission, $110 \mathrm{~K}$.

Figure 2.9: Residuals vs $D_{S O}$, the vertical distance between the $S$ adatom and the 'open' $\mathrm{Cu}$ atom in the second layer. The solid lines are cubic spline interpolations as guides to the eye. For ease of comparison all curves have been rescaled to have the value 1 at the minimum. Note the consistency in both the $\mathrm{k}$-space fitting and the R-space fitting and that the centroid of the minima has shifted from the position of the centroid in Figure 2.8.

Symbols:

Filled Squares: Normal emission, $110 \mathrm{~K}$.

Filled Circles: Normal emission, $155 \mathrm{~K}$.

Open Circles: $40^{\circ}$ Off normal emission, $140 \mathrm{~K}$.

Open Squares: $50^{\circ}$ Off normal emission, $110 \mathrm{~K}$. 
Figure 2.10: Residuals vs $D_{S A}$, the vertical distance between the $S$ adatom and the 'anti-covered' $\mathrm{Cu}$ atom in the second layer. The solid lines are cubic spline interpolations as guides to the eye. For ease of comparison all curves have been rescaled to have the value 1 at the minimum. Note the general consistency within each set of curves, and that the centroids of these minima are shifted still further.

Symbols:

Filled Squares: Normal emission, $110 \mathrm{~K}$.

Filled Circles: Normal emission, $155 \mathrm{~K}$.

Open Circles: $40^{\circ}$ Off normal emission, $140 \mathrm{~K}$.

Open Squares: $50^{\circ}$ Off normal emission, $110 \mathrm{~K}$.

Figure 2.11: Residuals vs $D_{\mathrm{S} 3}$, the vertical distance between the $\mathrm{S}$ adatom and the third $\mathrm{Cu}$ layer. The solid lines are cubic spline interpolations as guides to the eye. For ease of comparison all curves have been rescaled to have the value 1 at the minimum. The normal emission curves give a precise and reproducible value for this parameter, whereas the off-normal emission curves are relatively insensitive to this parameter.

Symbols:

Filled Squares: Normal emission, $110 \mathrm{~K}$.

Filled Circles: Normal emission, $155 \mathrm{~K}$.

Open Circles: $40^{\circ}$ Off normal emission, $140 \mathrm{~K}$.

Open Squares: $50^{\circ}$ Off normal emission, $110 \mathrm{~K}$. 
Figure 2.12: Residuals vs S-Cu Bond Length. The solid lines are cubic spline interpolations as guides to the eye. For ease of comparison all curves have been rescaled to have the value 1 at the minimum. The off-normal emission data give more precise estimates for this parameter than do the normal emission curves.

Symbols:

Filled Squares: Normal emission, $110 \mathrm{~K}$.

Filled Circles: Normal emission, $155 \mathrm{~K}$.

Open Circles: $40^{\circ}$ Off normal emission, $140 \mathrm{~K}$.

Open Squares: $50^{\circ}$ Off normal emission, $110 \mathrm{~K}$.

Figure 2.13: Comparison of Fourier filtered $(2 \AA<R<11 \AA$ ) normal emission $\chi(\mathrm{E})$ curves taken at $110 \mathrm{~K}$ (this work) and $300 \mathrm{~K}$ (reference 2 ).

Figure 2.14: Fit to $300 \mathrm{~K}$ normal emission data from reference 2.

Symbols:

Crosses: Best k-space fit.

Circles: Data. 
Figure 2.1:

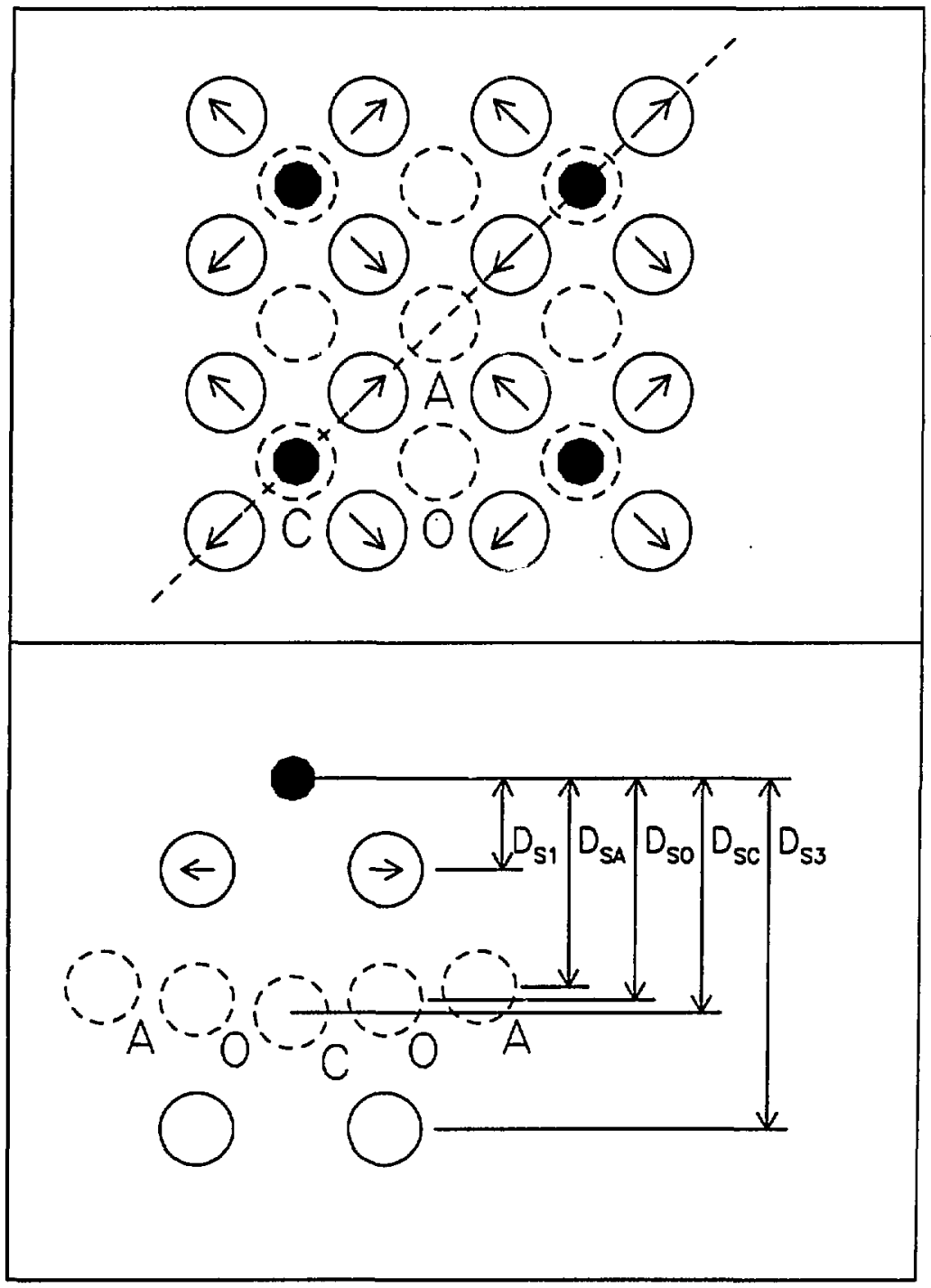




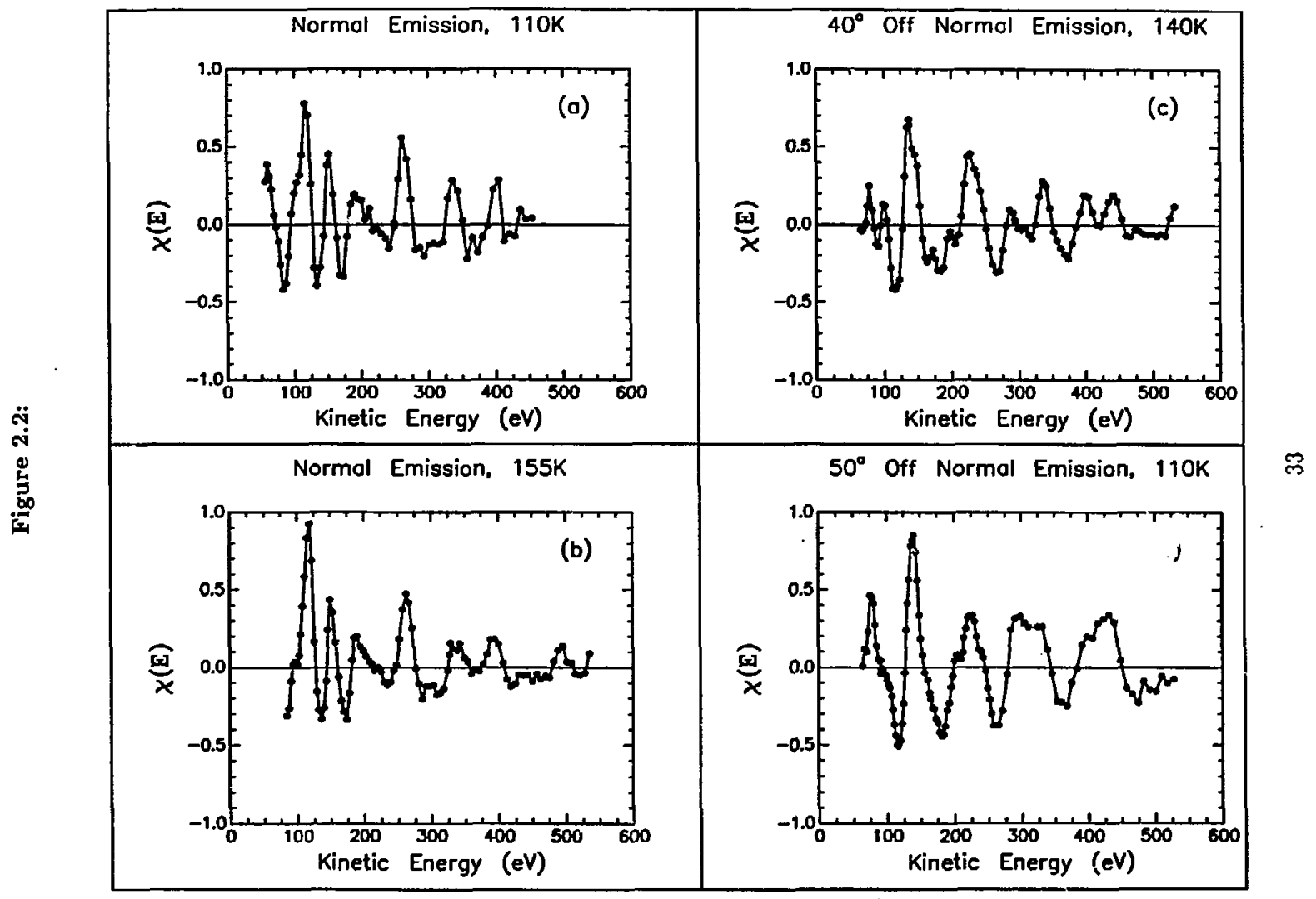


Figure 2.3:

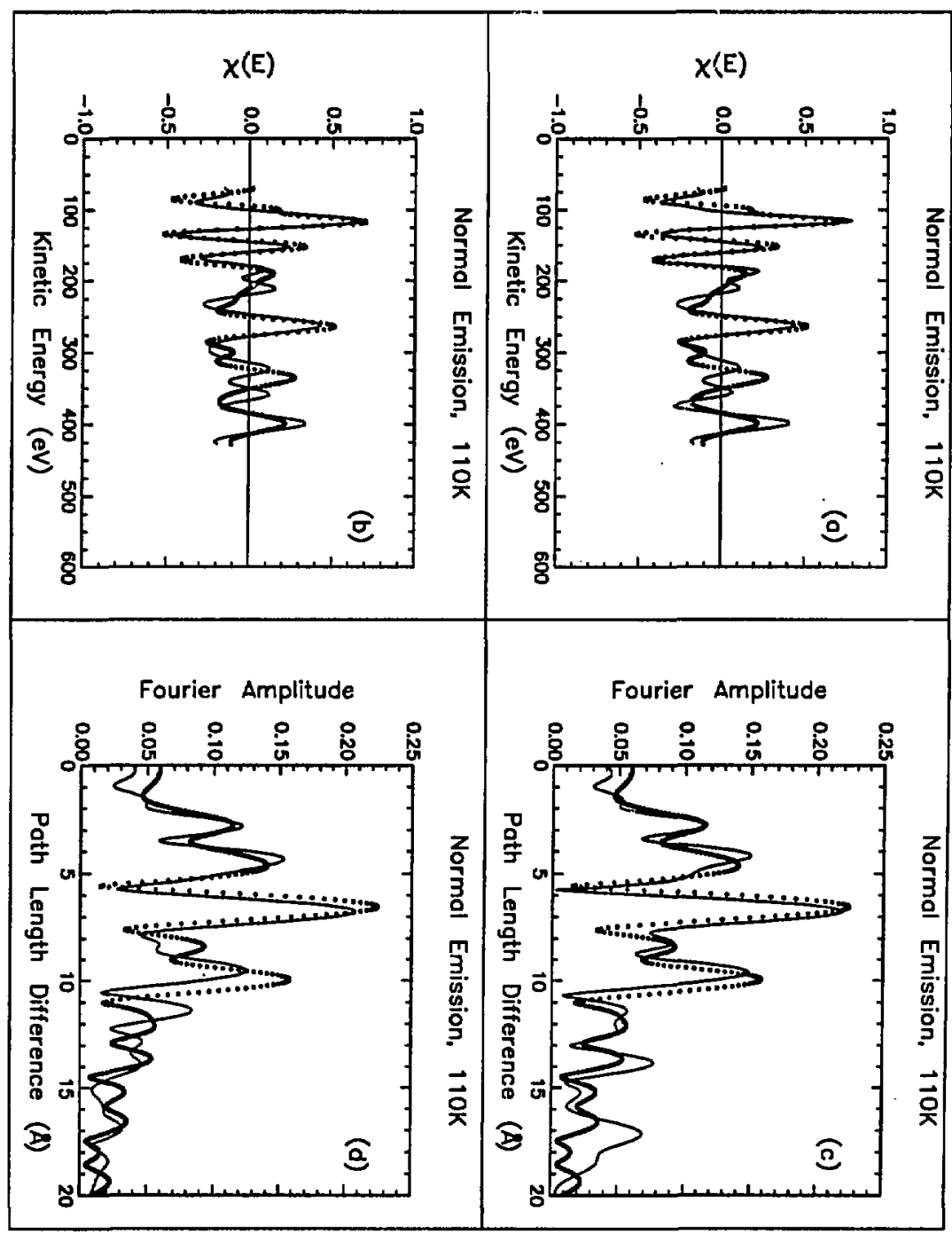


Figure 2.4:

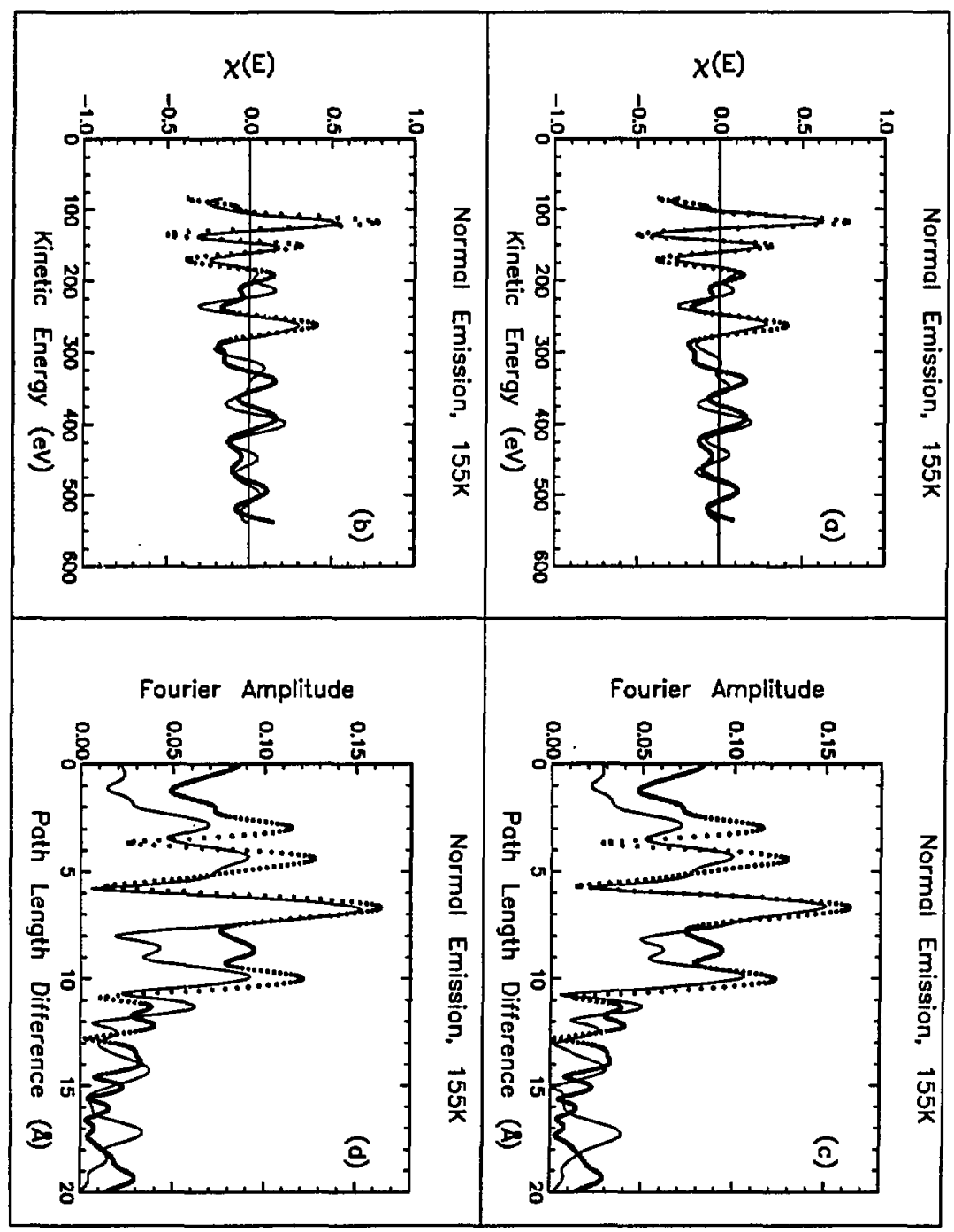


Figure 2.5:

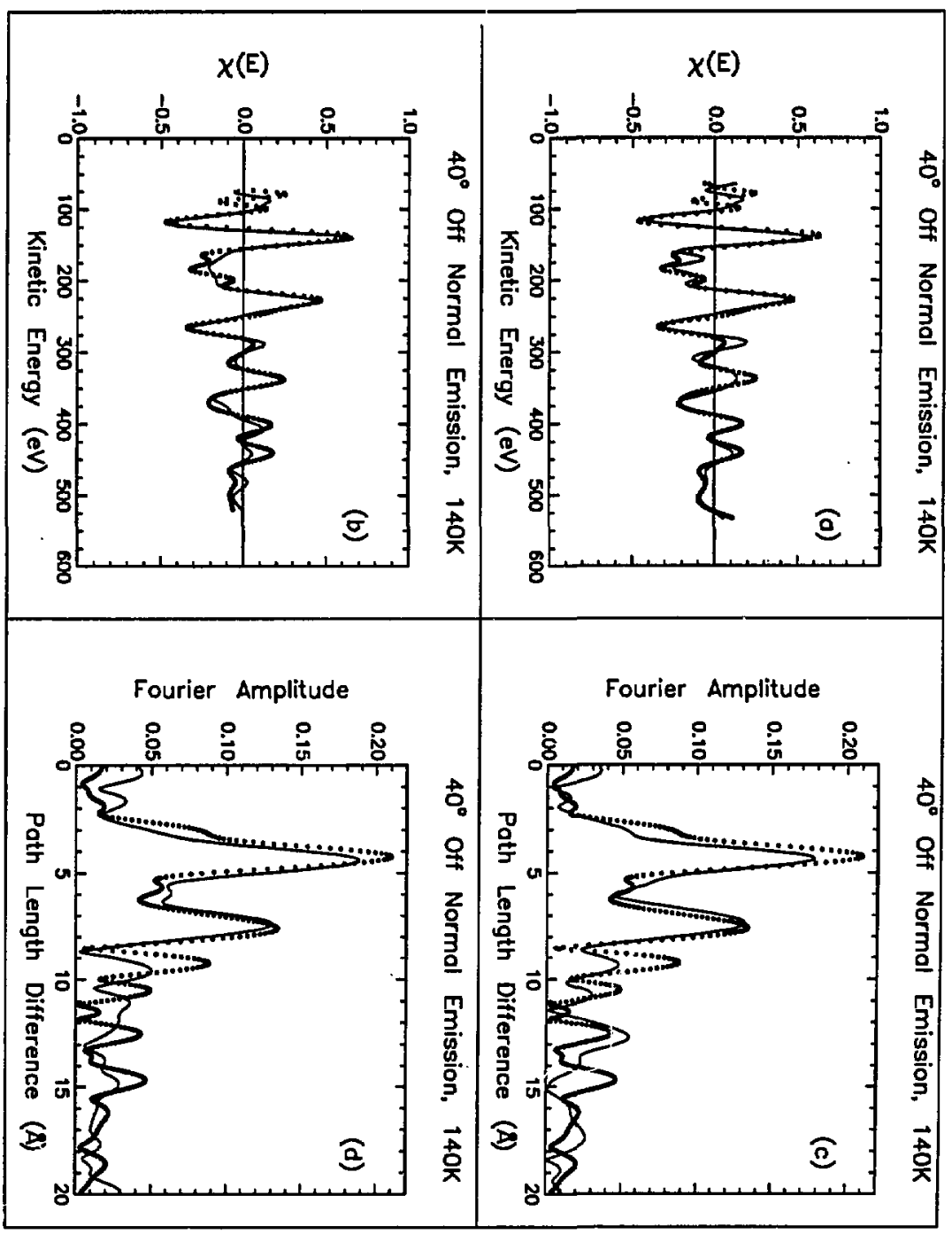


Figure 2.6:

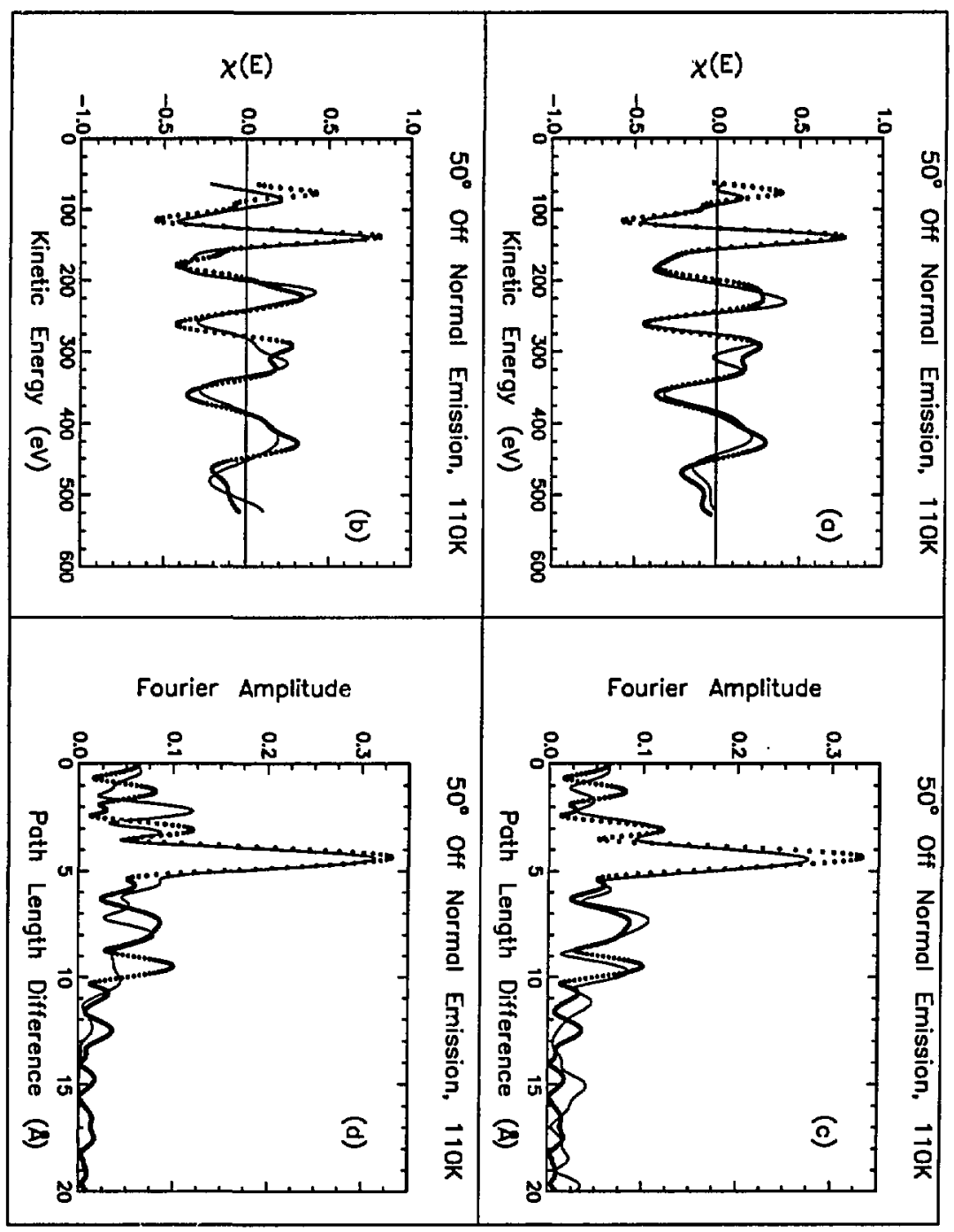


Figure 2.7:

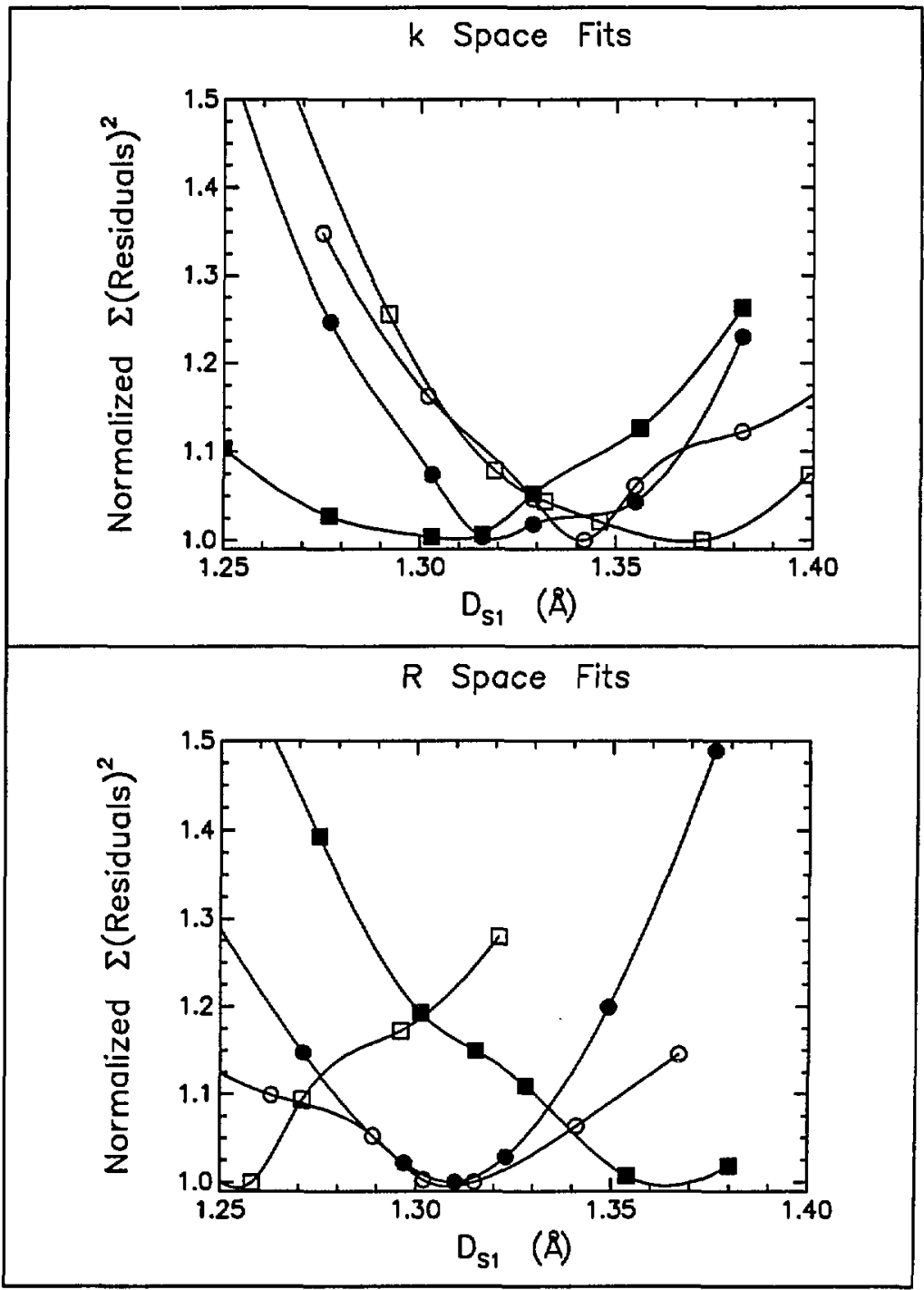


Figure 2.8:

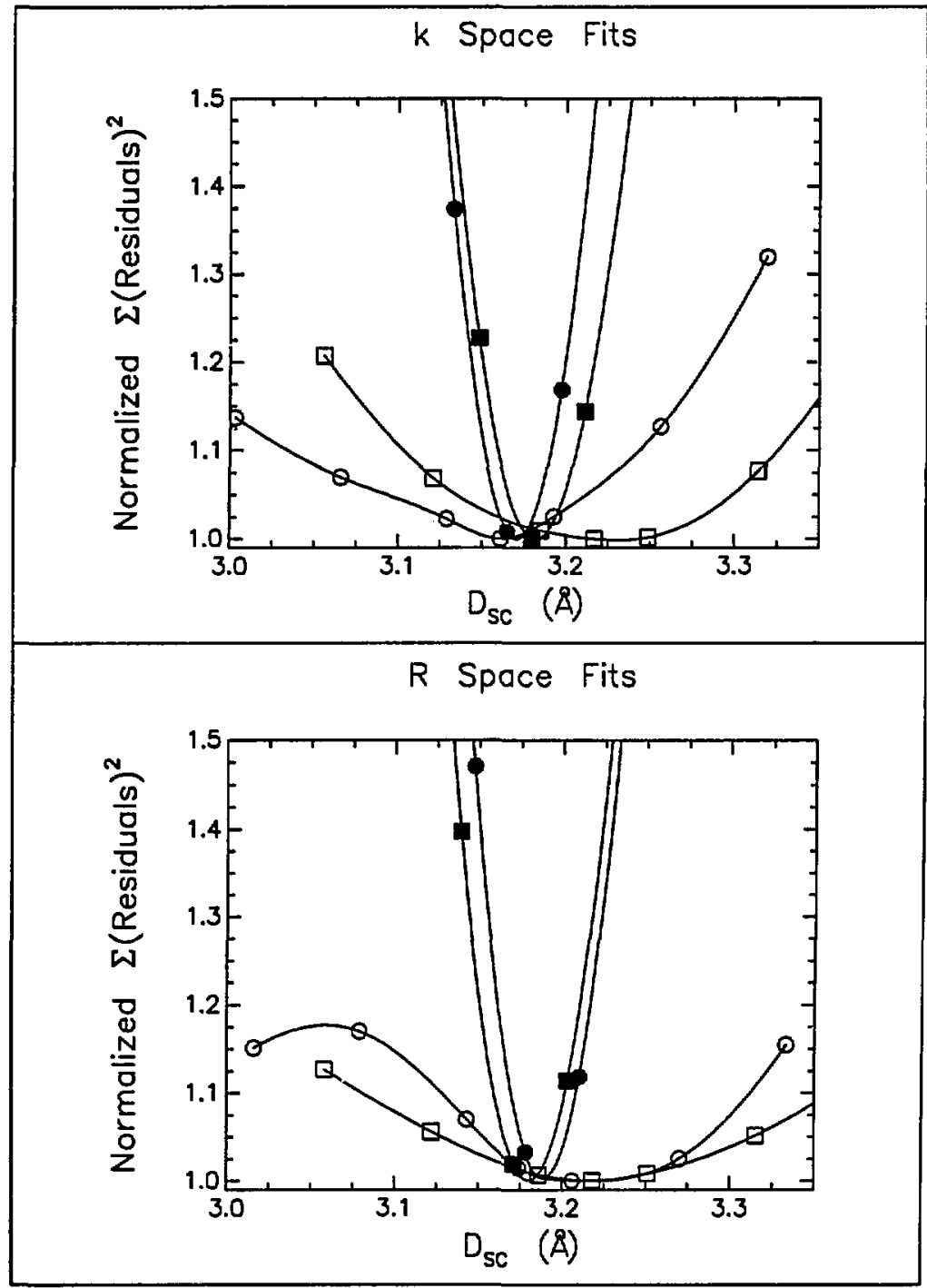


Figuĩe 2.9:

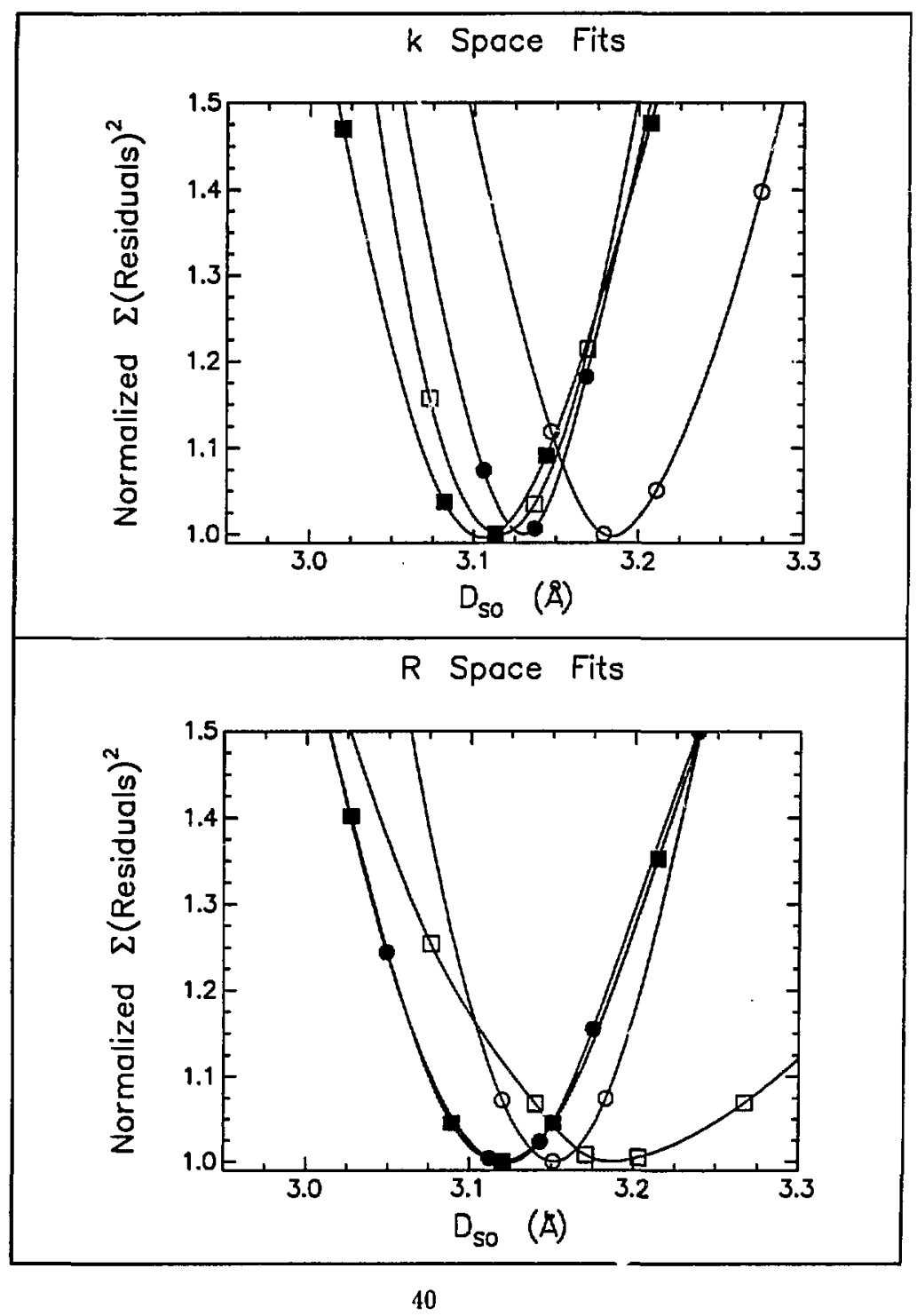




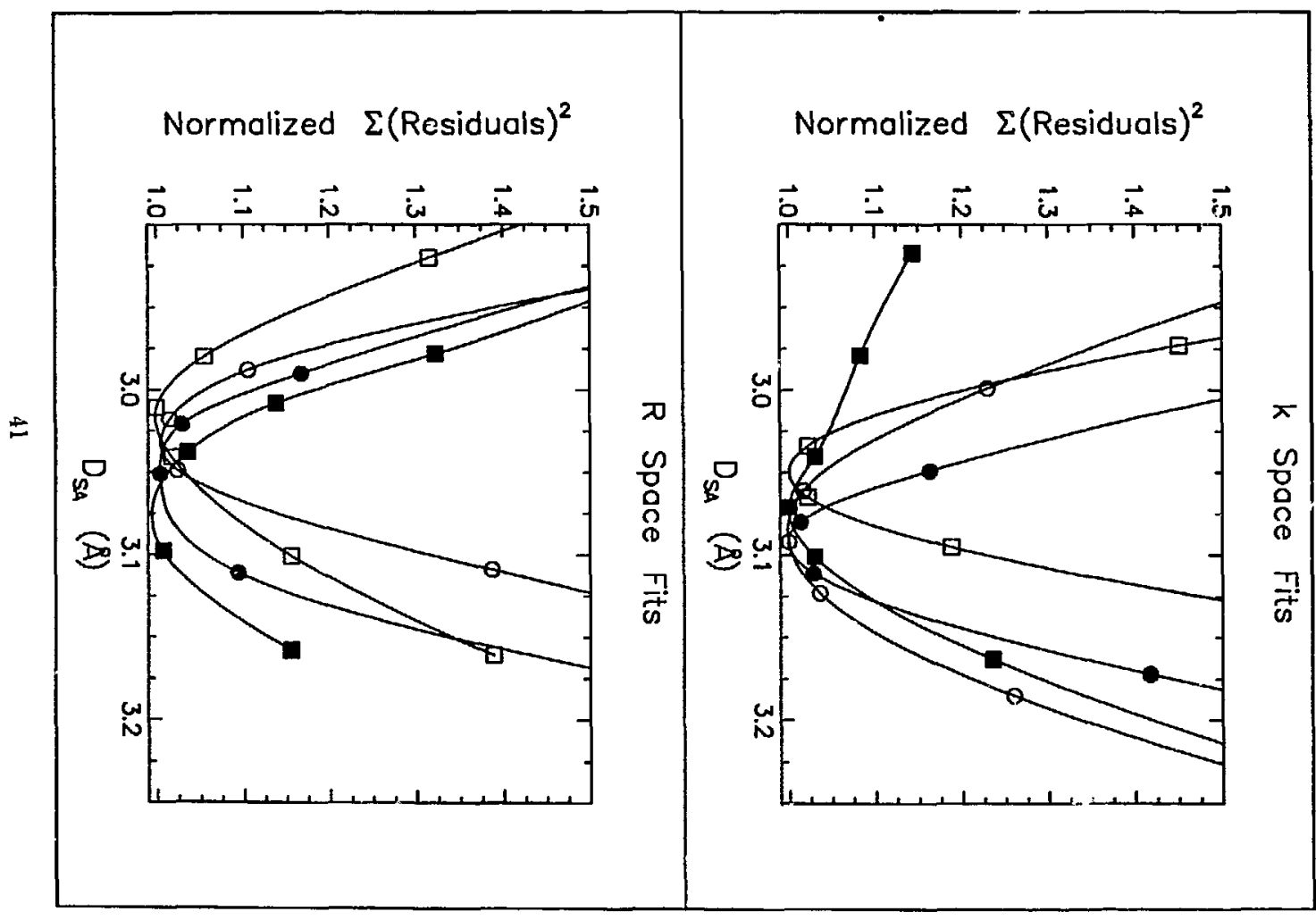


Figure 2.11:

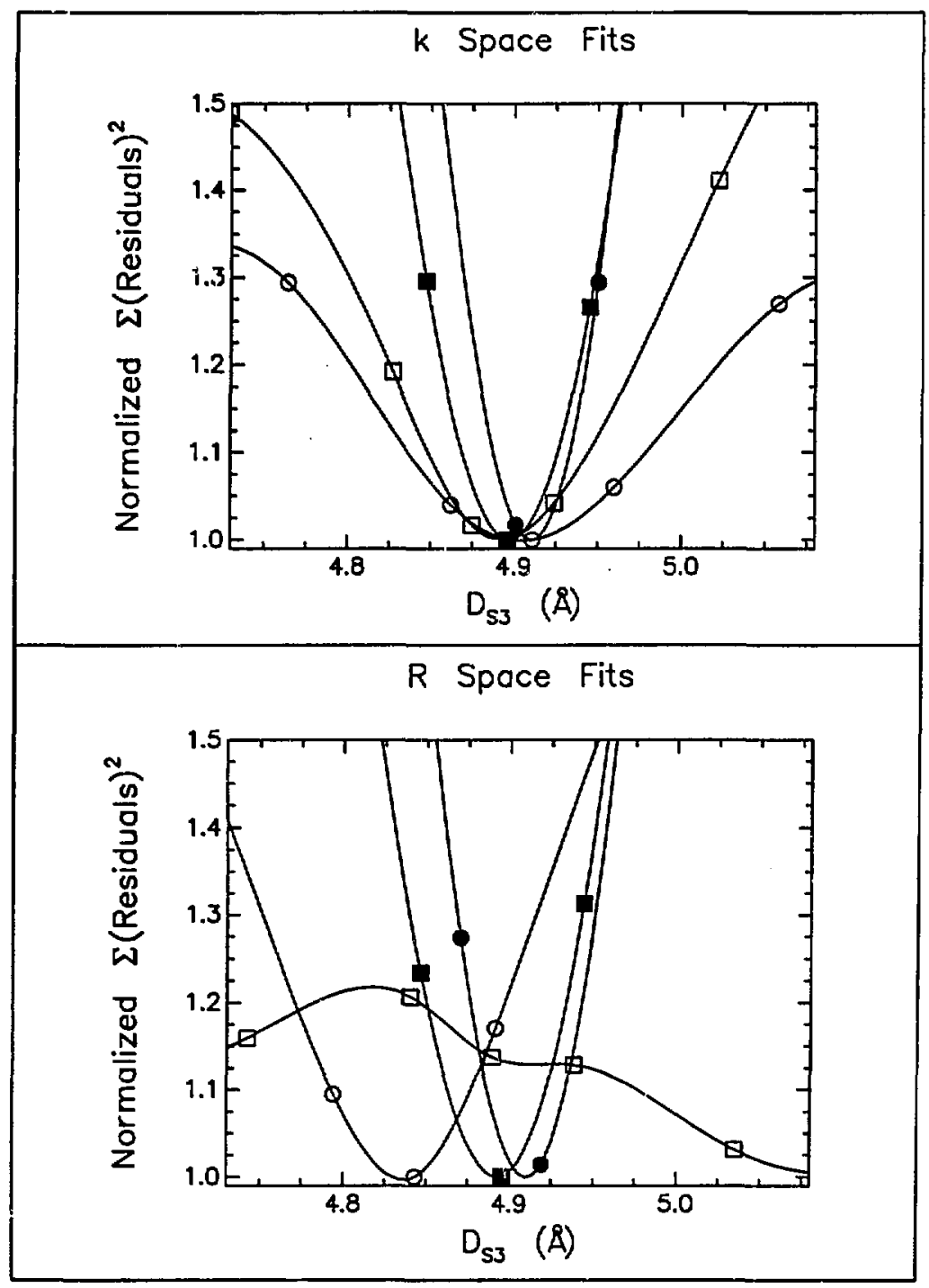


Figure 2.12:

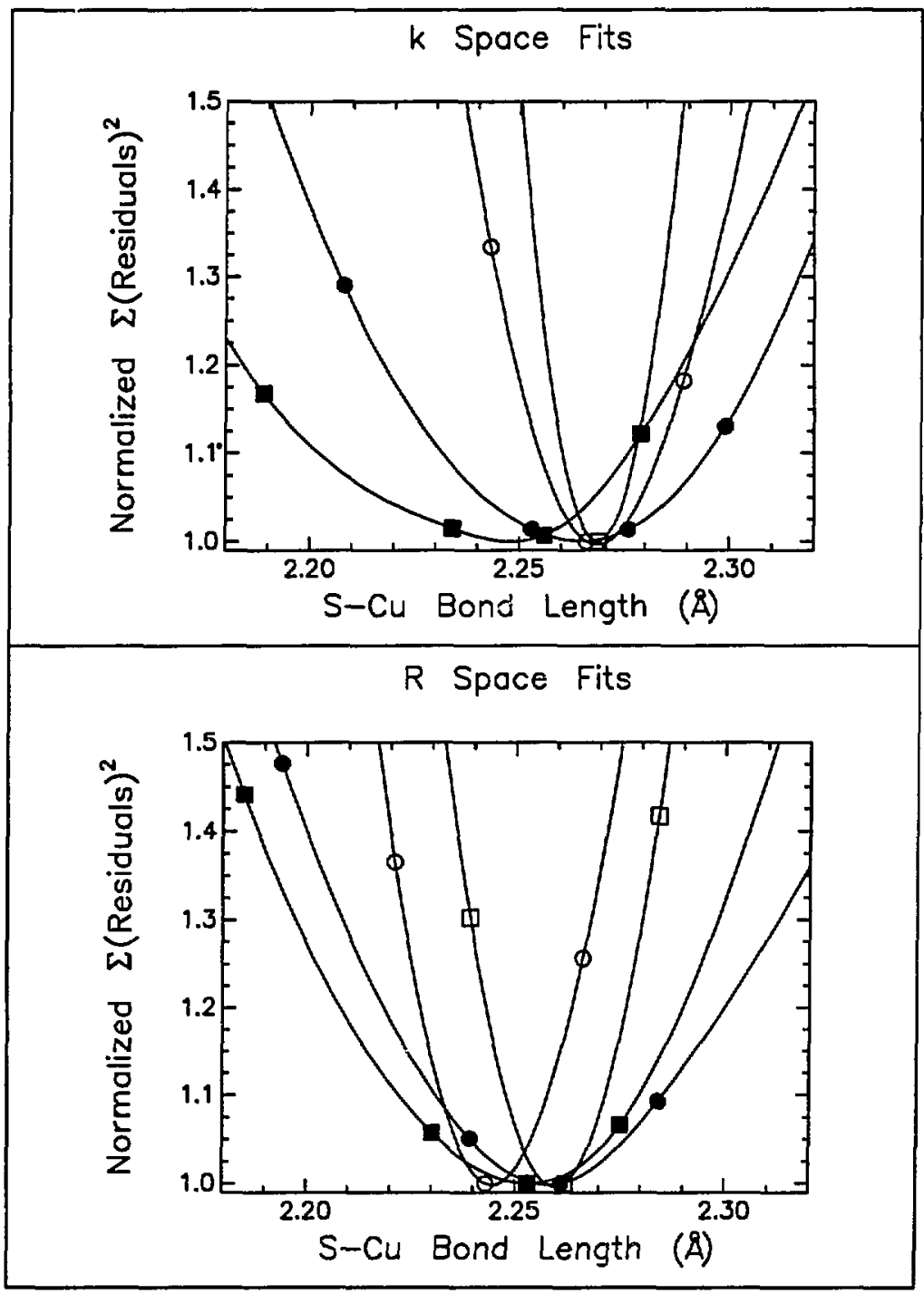


Figure 2.13:

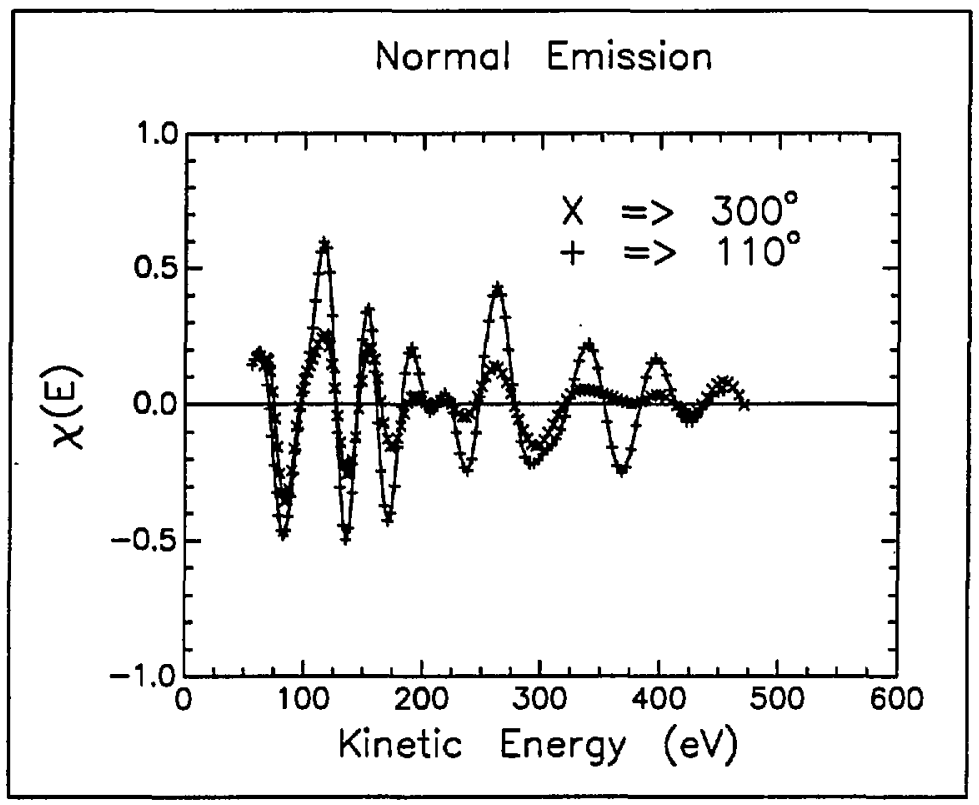


Figure 2.14:

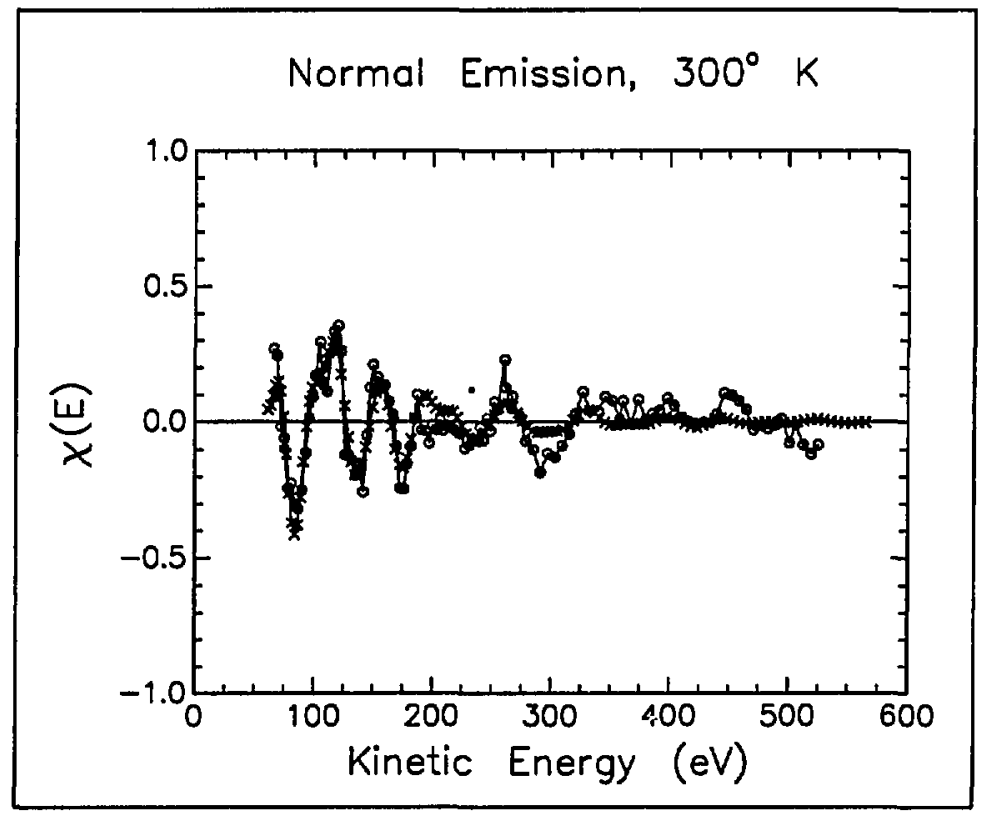




\section{Chapter 3}

\section{S/Ni(111) ARPEFS}

\subsection{Abstract}

We have performed low temperature (200 K) Angle Resolved Photoemission Extended Fine Structure studies of $\mathrm{p}(2 \times 2) \mathrm{S} / \mathrm{Ni}(111)$ and $(\sqrt{3} \times \sqrt{3}) \mathrm{R} 30^{\circ} \mathrm{S} / \mathrm{Ni}(111)$. Analysis of these low-temperature data using Multiple Scattering Spherical Wave calculations indicates that for $\mathrm{p}(2 \times 2) \mathrm{S} / \mathrm{Ni}(111) \mathrm{S}$ adsorbs onto the FCC threefold hollow site $1.53 \AA$ above the $\mathrm{Ni}$ surface. The $\mathrm{S}-\mathrm{Ni}$ bond length is determined to be $2.13 \AA$, indicating an outwards shift of the first layer Ni atoms.

\subsection{Introduction}

$\mathrm{S}$ forms several different ordered overlayers on $\mathrm{Ni}(111)$ depending on the degree of coverage, such as $\mathrm{p}(2 \times 2) \mathrm{S} / \mathrm{Ni}(111)^{41-50}$ at 0.25 monolayer, $(\sqrt{3} \times \sqrt{3}) \mathrm{R} 30^{\circ} \mathrm{S} / \mathrm{Ni}(111)^{43,49,50,51}$ at 0.33 monolayer, and the $(5 \sqrt{3} \times 2)^{44,50,52,53}$ at 0.4 monolayer. At higher coverages ( $>0.4$ monolayer) additional structures are found ${ }^{44,46,49,50,54}$. The $\mathrm{p}(2 \times 2) \mathrm{S} / \mathrm{Ni}(111)$ structure is the perhaps best understood of these. There is general agreement that the $\mathrm{S}$ adatom sits in a threefold hollow site. Those studies which could differentiate between the HCP and FCC site opted for the FCC site (i.e., the S continues the normal ABC stacking pattern of 
an FCC crystal). There is less agreement about the detailed structural parameters describing this site, however. The $\mathrm{S}-\mathrm{Ni}$ bond length has a reported range of $2.02 \AA<\mathrm{D}_{\mathrm{S}-\mathrm{Ni}}<2.20 \AA$. The distance between the $\mathrm{S}$ adatom and the outermost $\mathrm{Ni}$ layer has been reported as being $1.40 \AA<D_{\mathrm{S} 1}<1.66 \AA$. Low Energy Electron Diffraction (LEED) evidence suggests a horizontal expansion of the first surface layer. A summary of published $\mathrm{p}(2 \times 2) \mathrm{S} / \mathrm{Ni}(111)$ structures is given in Table 3.1 and a diagram of this overlayer is given in Figure 3.1.

There is uncertainty regarding the surface structure of $(\sqrt{3} \times \sqrt{3}) \mathrm{R} 30^{\circ} \mathrm{S} / \mathrm{Ni}(111)$. A photoemission study $^{43}$ was interpreted to show that in this structure the $S$ adatom shifts to an atop site. There has been little other investigation.

The $(5 \sqrt{3} \times 2)$ at 0.40 monolayer has been studied using SEXAFS ${ }^{4 / 4}$ and LEED. it is believed that the overlayer reconstructs to form a pseudo-c $(2 \times 2) \mathrm{S} / \mathrm{Ni}(100)$ surface. The S-Ni bond length for this structure is given as $2.27 \AA$. Higher coverages of $\mathrm{S} / \mathrm{Ni}(111)$ are believed to involve similar reconstructions.

Only the first of these overlayer structures would be thought a priori to be readily amenable to study using ARPEFS. In the LEED ${ }^{41}$ structure for $\mathrm{p}(2 \times 2) \mathrm{S} / \mathrm{Ni}(111)$ there is only one adsorption site: the threefold hollow FCC site. According to the LEED analysis there is also a slight horizontal displacement of the atoms in the first $\mathrm{Ni}$ layer. There was also found an increase in the layer separations between the first and second $\mathrm{Ni}$ layers and between the second and third Ni layers. ARPEFS data of such single domain adsorption systems has been found to be analyzable for many systems.

The $\mathrm{p}(2 \times 2) \mathrm{S} / \mathrm{Ni}(111)$ system is interesting to us for several other reasons. First, there is the detailed LEED ${ }^{41}$ study of this structure indicating reconstruction of the near surface layers. Comparison of the LEED ${ }^{41}$ result with the ARPEFS 
result might possibly show systematic differences between the two methods. We note that the LEED ${ }^{41}$ study was done by the same group that performed the detailed LEED analysis of $\mathrm{p}(2 \times 2) \mathrm{S} / \mathrm{Cu}(001)$. It further serve as a useful control experiment for the $(\sqrt{3} \times \sqrt{3}) \mathrm{R} 30^{\circ} \mathrm{S} / \mathrm{Ni}(111)$ overlayer, where there was some uncertainty over the adsorption site (atop versus 3-fold hollow, and if 3-fold hollow, which of the two possible sites).

In the published structures for $(5 \sqrt{3} \times 2) \mathrm{S} / \mathrm{Ni}(111)$, there are eight inequivalent $\mathrm{S}$ atoms in each unit cell. This fact, combined with the three domains possible for this overlayer, implies that $24 \chi(k)$ curves must be calculated for each trial structure. Given the number of curves needed in the analysis of the $\mathrm{S} / \mathrm{Cu}(001)$ data (one domain only), it was decided that this would be a prohibitive consiraint in data analysis. Therefore no attempt is made to study this or any of the higher coverages.

\subsection{Experimental}

A mechanically polished and chemically etched $\mathrm{Ni}(111)$ crystal was attached with tantalum strips to a tantalum sample plate, which was in turn mounted on a three axis manipulator equipped with $\mathrm{LN}_{2}$ cooling coils. Temperatures were measured using an unreferenced chromel-alumel thermocouple attached to the sample plate near the crystal. The manipulator was installed in a UHV chamber equipped with an ion sputtering gun, four grid LEED/Auger optics and a moveable hemispherical analyzer ${ }^{21,22}$. The crystal was cleaned by repeated cycles of $\mathrm{Ar}^{+}$sputtering $\left(0.5 \mathrm{kV}, 10^{-5}\right.$ torr $\left.\mathrm{Ar}^{+}\right)$and annealing to $570 \mathrm{C}$ until sharp $(1 \times 1)$ LEED patterns were obtained and S, $\mathrm{C}$, and $\mathrm{O}$ impurities were less than $1 \%$, according to Auger spectroscopy. Ambient dosing of the clean crystal with $1.3 \mathrm{~L}\left(130 \mathrm{sec} .1 \times 10^{-8}\right.$ torr) $\mathrm{H}_{2} \mathrm{~S}$ at $200 \mathrm{~K}$ produced a sharp $\mathrm{p}(2 \times 2)$ overlayer. Additional dosing $(2 \mathrm{~L}$ 
total) produced a $(\sqrt{3} \times \sqrt{3}) \mathrm{R} 30^{\circ} \mathrm{S} / \mathrm{Ni}(111)$ overlayer.

Sulfur 1s ARPEFS data were taken using the double crystal monochromator with $\mathrm{Ge}(111)$ crystals at Beamline X-24A at the National Synchrotron Light Source ${ }^{24}$. Photoemission spectra were taken in the $90 \mathrm{eV}-400 \mathrm{eV}\left(\mathrm{k}=4.4 \AA^{-1}\right.$ to $10 \AA^{-1}$ ) kinetic energy range using $2565 \mathrm{eV}-2875 \mathrm{eV}$ photons. The analyzer was operated at $160 \mathrm{eV}$ pass energy, giving an estimated overall energy resolution of $\sim 2 \mathrm{eV}$. The chamber base pressure was $3 \times 10^{-10}$ torr.

Sulfur ls photoemission spectra were taken in $0.07 \AA^{-1}$ to $0.1 \AA^{-1}$ increments over the energy range specified above. A total of 90 to 100 such spectra thus constitute a complete $\chi(k)$ curve. The ARPEFS data are reduced in the standard fashion ${ }^{4}$. Each individual S ls photoemission spectrum was fitted as a sum of an empirical background function, a Voigt function, and a Gaussian broadened step function. In keeping with previous work ${ }^{4}$ the Lorentzian portion of the Voigt function was fixed at $0.8 \mathrm{eV}$. The means and Gaussian widths of the Voigt function and of the step function are constrained to be equal. Thus, each individual photoemission spectrum is described by five parameters: the area of the Voigt function, the height of the step function, the mean and Gaussian width of the Voigt function, and the scale factor applied to the empirical background function. We then construct an $\mathrm{I}(\mathrm{E})$ curve by plotting the area of the Voigt function as a function of the Voigt mean, using the scaling factor of the empirical background to normalize each spectrum. In an EXAFS like manner we fit a quadratic or cubic polynomial $I_{0}$ to these raw $\mathrm{I}(\mathrm{E})$ curves and form a $\chi(E)$ curve using $\mathrm{I}_{\mathrm{t}}(\mathrm{E})=[1+\chi(\mathrm{E})] \mathrm{I}_{0}(\mathrm{E})$.

\subsection{Discussion}

We calculate theoretical $x(k)$ curves using the MSSW method described elsewhere ${ }^{25}$. Briefly, this model for ARPEFS calculates the interference between 
the primary photoelectron wave and the photoelectron waves scattered by atoms in the substrate. The method uses spherical waves and models the thermal vibration of the adsorbate and substrate atoms using a correlated Debye model. Effects of the finite angular acceptance of the analyzer and inelastic mean free path are also included.

Phase shifts were calculated on a superposition potential ${ }^{29}$ using modified programs by Loucks ${ }^{30}$, using free atom wavefunctions ${ }^{31}$. The phaseshifts for sulfur were based on a hypothetical bcc lattice with lattice constant $2.26 \AA$. The exchange potential was modelled using the Slater $\mathrm{X} \alpha$ approximation, with $\alpha=0.77$.

Presumably because of the $3 \times 10^{-10}$ torr base chamber pressure, it was found that the sample LEED pattern became degraded at the end of some of the $\chi(E)$ curves. Because of this and because of loss of temperature stability, the $\chi(E)$ curves had to be taken rapidly and over a shorter k-range than desirable. As a result of the experimental difficulties for the $\mathrm{p}(2 \times 2) \mathrm{S} / \mathrm{Ni}(111)$ system we have only two off normal emission curves, one at $35^{\circ}$ off normal (at $195 \pm 2 \mathrm{~K}$ ) and one at $50^{\circ}$ off normal (at $196 \pm 2 \mathrm{~K}$ ). For the $(\sqrt{3} \times \sqrt{3}) \mathrm{R} 30^{\circ} \mathrm{S} / \mathrm{Ni}(111)$ system the situation is slightly better, there is a normal emission curve (at $155 \pm 7 \mathrm{~K}$ ) and several off normal emission curves ( $35^{\circ}$ off normal, $215 \pm 2 \mathrm{~K}, 50^{\circ}$ off normal, $140 \pm 4 \mathrm{~K}, 50^{\circ}$ off normal, $220 \pm 1 \mathrm{~K}$ ). While these curves are not ideal for a solid structural assignment on either system, the curves do yield some consistent structural information for each system. The analysis follows.

\section{$3.4 .1 p(2 \times 2) S / N i(111)$}

These curves were subjected to the numerical analysis used in for $\mathrm{p}(2 \times 2) \mathrm{S} / \mathrm{Cu}(001)$, that is, fitting of the Fourier filtered data in both wavevector and real space. The curves were fitted with three structural parameters: $D_{S 1}$, 
the $\mathrm{S}$ to first layer $\mathrm{Ni}$ vertical distance, $\mathrm{D}_{\mathrm{S} 2}$, the $\mathrm{S}$ to second layer $\mathrm{Ni}$ vertical distance, and the $\mathrm{S}-\mathrm{Ni}$ bond length. Any radial expansion or contraction of the first-layer $\mathrm{Ni}$ atoms is thus implicitly defined by $\mathrm{D}_{\mathrm{S}_{1}}$ and th $\mathrm{S}-\mathrm{Ni}$ bond length. While the LEED study did use a fourth adjustable structural parameter, $D_{\mathrm{S} 3}$, the $\mathrm{S}$ to third layer $\mathrm{Ni}$ distance, it was felt that this would not be purposeful in the present case. Experience gained in fitting $\mathrm{S} / \mathrm{Cu}(001)$ data showed that off normal emission curves do not give very precise values for interlayer separations, and of the two curves, the one more likely to be sensitive to interlayer separations is incomplete. We assume the first and second $\mathrm{Ni}$ layers to be planar, even though there are two inequivalent atomic sites in each layer (in the first layer, $\frac{3}{4}$ of the $\mathrm{Ni}$ atoms are part of a threefold-hollow adsorption site and $\frac{1}{4}$ are not bonded to a $\mathrm{S}$ adsorivate. In the second layer, $\frac{1}{4}$ of the atoms are in under the so-called HCP threefold-hollow adsorption : $\quad$ I. Electrou emission angles and adsorbate and substrate surface Debye temperatures were treated as adjustable parameters. The bulk Debye temperature ${ }^{41}$ was set at $440 \mathrm{~K}$. The isotropic $\mathrm{Ni}$ surface and $\mathrm{S}$ adsorbate Debye temperatures were treated as adjustable parameters, being set initially to $310 \mathrm{~K}$ and $420 \mathrm{~K}$, respectively. Results of the fitting are given in Table 3.1 and Figures 3.3-3.4. Plots of R-factor curves are given in Figure 3.5.

\section{$35^{\circ}$ Off normal Emission}

The raw $\chi(\mathrm{E})$ curve is shown in Figure 3.2. We note that the low temperature has yielded strong oscillations, similar in strength to those seen in $\mathrm{p}(2 \times 2) \mathrm{S} / \mathrm{Cu}(001)$. The break in the $35^{\circ}$ off normal emission data is due to having stepped forwards rather than backwards one unit in $\mathrm{k}$ space after a computer problem. Lack of beam time prevented repeating this scan. Auto-regressive extrapolation of the low energy side indicates that the missing region is featureless and well approximated by using 
a cubic spline interpolation across the gap, so we proceed with the analysis. Both the R-space and k-space fits yield theoretical curves that track the Fourier filtered data fairly well. We note that fitting in $k$-space, however, yielded a theoretical curve which deviates from the data at $\sim 250 \mathrm{eV}$, though overall amplitude and phase in l:-space seem well matched (Figure 3.3a). The Fourier transforms (Figure 3.3c) disagree at $\sim 6 \AA$, but with the exception of the main peak in the Fourier transform the overall agreement appears acceptable. We note that this disagreement at $\sim 6 \AA$ persists even if the fitting is performed in R-space (Figure 3.3d). This R-space structure appears to give the better $k$-space fit (Figure $3.3 \mathrm{~b}$ ).

\section{$50^{\circ}$ Off normal Emission}

The $50^{\circ}$ off normal curve (Figure $3.2 \mathrm{~b}$ ) is dominated by a single frequency, indicating that this curve was probably taken along the $\mathrm{S}-\mathrm{Ni}$ bond. We note that the fits appear credible in both $R$ and $k$ space for both curves, though the $k$-space fit has apparently yielded the more credible agreement in both k-space and R-space.

\section{Summary, $p(2 \times 2) S / N i(111)$}

We note further that our results are in general agreement with the LEED study, at least as far as the first layer is concerned. Our data indicate an outwards expansion of the first layer $\mathrm{Ni}$ atoms. The bond length is in the middle of the range of available values, as is the $\mathrm{S}-\mathrm{Ni}$ vertical distance. In contrast to the LEED result, our data do not show any expansion of the first to second $\mathrm{Ni}$ layer distance. We note that similar to the results of $\mathrm{p}(2 \times 2) \mathrm{S} / \mathrm{Cu}(001)$, ARPEFS gives a bond length $0.03 \AA$ longer than LEED and a $S$ to first layer substrate distance also $0.03 \AA$ longer than the LEED result. 


\subsection{2 $(\sqrt{3} \times \sqrt{3}) \mathrm{R} 30^{\circ} \mathrm{S} / \mathrm{Ni}(111)$}

We show raw $\chi(\mathrm{E})$ curves for $(\sqrt{3} \times \sqrt{3}) \mathrm{R} 30^{\circ} \mathrm{S} / \mathrm{Ni}(111)$ in Figure 3.6. We note that in two of the curves there is some normalization difficulty in the overlapped portions of the $50^{\circ}$ off normal curves. In the $140 \mathrm{~K}$ curve we had difficulty scaling sections of the curve at a break near $200 \mathrm{eV}$. In the $220 \mathrm{~K}$ curve there was a similar problem near $220 \mathrm{eV}$. The uncertainties in joining the sub-spectra, however, do not affect the main point of interest for these curves, namely, the smaller amplitude in the oscillations in these curves (roughly $50 \%$ ) compared to the oscillations for the $\mathrm{p}(2 \times 2) \mathrm{S} / \mathrm{Ni}(111)$ case. A quick TSMQNE calculation for $(\sqrt{3} \times \sqrt{3}) \mathrm{R} 30^{\circ} \mathrm{S} / \mathrm{Ni}(111)$ indicates that theoretically these oscillations are too small to correspond to a well ordered overlayer of $S$ atoms in FCC sites. In Figure 3.7 we show nor . al emission $(\sqrt{3} \times \sqrt{3}) \mathrm{R3} 0^{\circ} \mathrm{S} / \mathrm{Ni}(111)$ data with theoretical $\chi(\mathrm{k})$ curves corresponding to FCC and HCP adsorption sites. It is true that a change of site to the HCP threefold hollow site decreases the amplitude of the oscillations (since the lobe of the outgoing p-wave is no longer aligned with a backscattering atom). Looking at Figures 3.8 and 3.9, though, we see that the HCP site is ruled out by the off-normal emission curves. In particular, the $35^{\circ}$ off normal data rule out the HCP site (see Figure 3.8c). The phase of the oscillation in the data curve is opposite to that of the theory curve. The Fourier transform of the $220 \mathrm{~K} 50^{\circ}$ data (Figure $3.9 \mathrm{~d}$ ) also indicates that the HCP site is wrong. The data are dominated by a single frequency, but theory shows that such a site would contain a mixture of roughly equal intensity frequencies. It has been suggested ${ }^{43}$ that the $(\sqrt{3} \times \sqrt{3}) \mathrm{R} 30^{\circ} \mathrm{S} / \mathrm{Ni}(111)$ data may consist of atop adsorption sites. We have calculaied $\chi(\mathrm{E})$ curves for atop sites for a range of S-Ni vertical distances. We show theoretical $\chi(E)$ curves for a S-Ni vertical distance of $1.75 \AA$ (i.e. S-Ni bond length of $2.15 \AA$ ) in Figures $3.10-3.12$. We see that the normal emission 
curve rules out this geometry, judging by the amplitudes and phases of the $\chi(E)$ curves. We note that the amplitudes of the $35^{\circ}$ and $50^{\circ} \chi(\mathrm{E})$ curves also rule out the atop site. We furthermore calculated $\chi(E)$ curves for the bridged site. Figures $3.10-3.12$ show that the data curves also rule out this bonding geometry.

\subsubsection{Summary, $(\sqrt{3} \times \sqrt{3}) \mathrm{R} 30^{\circ} \mathrm{S} / \mathrm{Ni}(111)$}

We have calculated $\chi(E)$ curves for four high symmetry adsorption sites for $(\sqrt{3} \times \sqrt{3}) \mathrm{R30}$ S/Ni(111). We found that three of the sites are ruled out by the phase of the oscillations in at least one of the data $\chi(E)$ curves. The $35^{\circ}$ curve rules out the HCP site, the $50^{\circ}$ curve rules out the bridged site, and the normal emission curve rules out the atop site. We regard phase disagreement as being fairly compelling, since we expect surface disorder to reduce the amplitude of any measured oscillation, but not the phase. We note the the FCC site appears to be excluded primarily by disagreement between the amplitudes of the theory and data curves. This leaves us with several possible explanations:

(a) a disordered overlayer has been prepared, decreasing the apparent oscillation, and/or

(b) there were undetermined experimental errors leading to a decrease in the apparent oscillation, and/or

(c) there is something wrong with the TSMQNE theory, as applied to $\mathrm{S} / \mathrm{Ni}(111)$, and/or

(d) if the adsorption site is unique, it is not a high-symmetry site, and/or

(e) we have a mixture of adsorption sites, none of which is dominant enough to appear to be the sole structure. 
The success in fittin - the $\mathrm{p}(2 \times 2) \mathrm{S} / \mathrm{Ni}(111)$ data would seem to rule out reasons $\mathrm{a}, \mathrm{b}$ and $\mathrm{c}$. Given that the recipe for preparing $(\sqrt{3} \times \sqrt{3}) \mathrm{R} 30^{\circ} \mathrm{S} / \mathrm{Ni}(111)$ differs from the recipe used to make $\mathrm{p}(2 \times 2) \mathrm{S} / \mathrm{Ni}(111)$ only in the amount of dosing with $\mathrm{H}_{2} \mathrm{~S}$, it is unlikely that half of the adsorbate atoms would be in disordered positions, especially since there is only a $25 \%$ difference in coverage. Thus, we see no compelling reason to invoke explanation (a). We also see no compelling reason to invoke (b), again given the relative success in modelling the $\mathrm{p}(2 \times 2) \mathrm{S} / \mathrm{Ni}(111)$ data. We point out that the $\mathrm{p}(2 \times 2) \mathrm{S} / \mathrm{Ni}(111)$ data were taken in the middle of the $(\sqrt{3} \times \sqrt{3}) \mathrm{R} 30^{\circ} \mathrm{S} / \mathrm{Ni}(111)$ data. Thus any experimental problem would have had to disappear during the sample change and then reappear once the $(\sqrt{3} \times \sqrt{3}) \mathrm{R} 30^{\circ} \mathrm{S} / \mathrm{Ni}(111)$ series of curves was continued. Reason (c) we exclude, again given the success in fitting the $\mathrm{p}(2 \times 2) \mathrm{S} / \mathrm{Ni}(111)$ data. Reasons $\mathrm{a}$ and $\mathrm{b}$ refer to random mistakes. It is quite possible that the recipe used to prepare $(\sqrt{3} \times \sqrt{3}) \mathrm{R} 30^{\circ} \mathrm{S} / \mathrm{Ni}(111)$ is consistently giving the same overlayer, which is not a high-symmetry site. We exclude this reason because we were not able to find a unique adsorption site which would reproduce the data. Atop and bridged sites were modelled, without success. A brute-force search of overlayer registry was also unconvincing. This technique had been invoked in an ARPEFS study of $\mathrm{PH}_{x} / \mathrm{Ge}(111)$. In the present case this was done using a simplex search with three structural parameters, the $x, y$, and $z$ coordinates of the $S$ overlayer as variables. Domain averaging was included. This resulted in the favored location of the $\mathrm{S}$ adatom being slightly off the center of the threefold hollow site, but again the quality of the fit was visually displeasing. We interpret the slightly off center site fo : : single-site $(\sqrt{3} \times \sqrt{3}) \mathrm{R} 30^{\circ} \mathrm{S} / \mathrm{Ni}(111)$ to mean not that this is the real site, but that the minimization routine favors the threefold site, but is trying to decrease the backscattering strength the the nearest $\mathrm{Ni}$ atoms. This is done by removing 
the alignment of the adatom and the crystal plane. Given that we cannot think of any compelling reason why the experiment should be invalid and that we think the TSMQNE code should be valid for $\mathrm{S} / \mathrm{Ni}(111)$, we are left with a dilemma.

\subsection{Conclusions}

We have taken ARPEFS spectra for $\mathrm{p}(2 \times 2) \mathrm{S} / \mathrm{Ni}(111)$ and $(\sqrt{3} \times \sqrt{3}) \mathrm{R} 30^{\circ} \mathrm{S} / \mathrm{Ni}(111)$. The $\mathrm{p}(2 \times 2) \mathrm{S} / \mathrm{Ni}(111)$ data are consistent with the FCC adsorption site. Fitting the data in k-space and $\mathrm{R}$-space indicates a $\mathrm{S}-\mathrm{Ni}$ bond length of $2.13 \AA$ and a S-Ni vertical distance of $1.54 \AA$. These values indicate a radial displacement of the first-layer $\mathrm{Ni}$ atoms from the unreconsiructed bulk positions. We see no change in the interlayer spacings from the bulk positions.

We are unable to explain our $(\sqrt{3} \times \sqrt{3}) \mathrm{R} 30^{\circ} \mathrm{S} / \mathrm{Ni}(111)$ data.

\subsection{Acknowledgements}

This work was supported by the Director, Office of Energy Research, Office of Basic energy Sciences, Chemical Sciences Division of the U.S. Department of Energy under Contract No. DE-AC03-76SF00098. It was performed at the National Synchrotron Light Source, which is supported by the Department of Energy under Contract No. DE-AC02-76CH00016. We are deeply grateful to B. Karlin and D. Lindle for their assistance at NSLS. We also thank Dr.J. Stöhr for the Ni(111) crystal used in this experiment. 


\subsection{Tables}

Table 3.1: Summary of structural parameters for $\mathrm{p}(2 \times 2) \mathrm{S} / \mathrm{Ni}(111)$. Derived values are in italics. Uncertainties in the last digit of each parameter, where given, are in parentheses.

\begin{tabular}{lllccc}
\hline $\mathrm{D}_{\mathrm{S1}}$ & $\mathrm{D}_{\mathrm{S} 2}$ & $\mathrm{D}_{23}$ & Bond Length & $\Delta$ & Method \\
\hline 1.50 & 3.59 & 2.08 & 2.10 & +0.03 & LEED $^{41}$ \\
1.66 & 3.41 & 2.03 & $2.20(3)$ & & SEXAFS $^{42}$ \\
$1.69(02)$ & & & $2.23(2)$ & & SEXAFS $^{44}$ \\
$1.61(06)$ & & & $2.16(4)$ & & IS $^{45}$ \\
\hline
\end{tabular}

Table 3.2: Results of Fitting $\mathrm{p}(2 \times 2) \mathrm{S} / \mathrm{Ni}(111)$ data in $\mathrm{k}$-space and $\mathrm{R}$-space.

\begin{tabular}{|c|c|c|c|c|c|}
\hline \multirow[t]{2}{*}{ Parameter } & \multicolumn{2}{|c|}{$35^{\circ}$ Off Normal } & \multicolumn{2}{|c|}{$50^{\circ}$ Off Normal } & \multirow[t]{2}{*}{ Average V/alue } \\
\hline & R-Space & k-Space & R-Space & -Space & \\
\hline $\mathrm{D}_{\mathrm{S1}}$ & $1.617(16)$ & $1.556(34)$ & $1.507(12)$ & $1.496(22)$ & $1.540(29)$ \\
\hline $\mathrm{D}_{\mathrm{S2}}$ & $3.579(17)$ & $3.542(42)$ & $3.544(29)$ & $3.620(36)$ & $3.574(14)$ \\
\hline Bond & $2.160(06)$ & $2.139(13)$ & $2.133(06)$ & $2.132(06)$ & $2.141(07)$ \\
\hline$\Delta$ & $-0.006(20)$ & $+0.030(40)$ & $+0.072(15)$ & $+0.080(23)$ & $+0.049(32)$ \\
\hline$V_{0}$ & 11.1 & 15.0 & 12 & 15.0 & \\
\hline
\end{tabular}




\subsection{Figure Captions}

Figure 3.1: Schematic of $\mathrm{p}(2 \times 2) \mathrm{S} / \mathrm{Ni}(111)$, showing structure and label definitions.

a) Top view, showing outwards reconstruction of first layer $\mathrm{Ni}$ atoms.

b) Side view, showing layer separations.

Figure 3.2: Raw $\chi(E)$ Curves, $p(2 \times 2) S / N i(111)$.

(a) $35^{\circ}$ Off Normal Emission.

(b) $50^{\circ}$ Off Normal Emission.

Figure 3.3: Results of fitting the $\mathrm{p}(2 \times 2) \mathrm{S} / \mathrm{Ni}(111) 35^{\circ}$ off normal emission data in k-space and R-space. The $k$-space curves are Fourier filtered at $1.4 \AA<\mathrm{R}<14.4 \AA$.

(a) and (c) Comparison of data (dots) and theory (solid line) curves. Fit performed in $k$-space.

(b) and (d) Comparison of data (dots) and theory (solid line) curves. Fit performed in R-space.

Figure 3.4: Results of fitting the $\mathrm{p}(2 \times 2) \mathrm{S} / \mathrm{Ni}(111) 50^{\circ}$ off normal emission data in $k$-space and $R$-space. The $k$-space curves are Fourier filtered at $1.4 \AA<\mathrm{R}<14.4 \AA$.

(a) and (c) Comparison of data (dots) and theory (solid line) curves. Fit performed in k-space.

(b) and (d) Comparison of data (dots) and theory (solid line) curves. Fit performed in R-space. 
Figure 3.5: Residuals vs. structural parameters, $\mathrm{p}(2 \times 2) \mathrm{S} / \mathrm{Ni}(111)$. The solid lines are cubic spline interpolations as guides to the eye. For ease of comparison all curves have been rescaled to have the value 1 at the minimum.

Symbols:

Filled Squares: $35^{\circ}$ Off Normal Emission, R space.

Open Squares: $35^{\circ}$ Off Normal Emission, $k$ space.

Filled Circles: $50^{\circ}$ Off Normal Emission, $R$ space.

Open Circles: $50^{\circ}$ Off Normal Emission, $k$ space.
a) R-factor vs. $D_{S_{1}}$, the $S$ to first layer $\mathrm{Ni}$ vertical distance.
b) R-factor vs. $D_{S 2}$, the $S$ to second layer $\mathrm{Ni}$ vertical distance.
c) R-factor vs. S-Ni bond length.

Figure 3.6: Raw $\chi(E)$ Curves, $(\sqrt{3} \times \sqrt{3}) \mathrm{R} 30^{\circ} \mathrm{S} / \mathrm{Ni}(111)$.
(a) Normal Emission (155 K).
(b) $35^{\circ}$ Off Normal Emission (215 K).
(c) $50^{\circ}$ Off Normal Emission (148 K).
(d) $3 \mathrm{j}^{\circ}$ Off Normal Emission (220 K).

Figure 3.7: Comparison of normal emission $(\sqrt{3} \times \sqrt{3}) \mathrm{R} 30^{\circ} \mathrm{S} / \mathrm{Ni}(111)$ data with $\chi(E)$ curves calculated for unreconstructed FCC and HCP adsorption sites. A S-Ni vertical distance of $1.64 \AA$ is assumed.

(a) and (c) Comparison of data (dots) and FCC theory (solid line) curves. (b) and (d) Comparison of data (dots) and HCP theory (solid line) curves. 
Figure 3.8: Comparison of $35^{\circ}$ off normal emission $(\sqrt{3} \times \sqrt{3}) \mathrm{R} 30^{\circ} \mathrm{S} / \mathrm{Ni}(111)$ data with $\chi(E)$ curves calculated for unreconstructed FCC and HCP adsorption sites. A S-Ni vertical distance of $1.64 \AA$ is assumed.

(a) and (c) Comparison of data (dots) and FCC theory (solid line) curves.

(b) and (d) Comparison of data (dots) and HCP theory (solid line) curves.

Figure 3.9: Comparison of $50^{\circ}$ off normal emission $(\sqrt{3} \times \sqrt{3}) \mathrm{R} 30^{\circ} \mathrm{S} / \mathrm{Ni}(111)$ data with $\chi(E)$ curves calculated for unreconstructed FCC and HCP adsorption sites. A S-Ni vertical distance of $1.64 \AA$ is assumed.

(a) and (c) Comparison of data (dots) and FCC theory (solid line) curves.

(b) and (d) Comparison of data (dots) and HCP theory (solid line) curves.

Figure 3.10: Comparison of normal emission $(\sqrt{3} \times \sqrt{3}) \mathrm{R} 30^{\circ} \mathrm{S} / \mathrm{Ni}(111)$ data with $\chi(\mathrm{E})$ curves calculated for unreconstructed bridged and atop adsorption sites. A S-Ni bond length of $2.15 \AA$ is assumed.

(a) and (c) Comparison of data (dots) and bridged site theory (solid line) curves.

(b) and (d) Comparison of data (dots) and atop site theory (solid line) curves.

Figure 3.11: Comparison of $35^{\circ}$ off normal emission $(\sqrt{3} \times \sqrt{3}) \mathrm{R} 30^{\circ} \mathrm{S} / \mathrm{Ni}(111)$ data with $\chi(E)$ curves calculated for unreconstructed bridged and atop adsorption sites. A S-Ni bond length of $2.15 \AA$ is assumed.

(a) and (c) Comparison of data (dots) and bridged site theory (solid line) curves.

(b) and (d) Comparison of data (dots) and atop site theory (solid line) curves. 
Figure 3.12: Comparison of $50^{\circ}$ off normal emission $(\sqrt{3} \times \sqrt{3}) \mathrm{R} 30^{\circ} \mathrm{S} / \mathrm{Ni}(111)$ data with $\chi(E)$ curves calculated for unreconstructed bridged and atop adsorption sites. A S-Ni bond length of $2.15 \AA$ is assumed.

(a) and (c) Comparison of data (dots) and bridged site theory (solid line) curves.

(b) and (d) Comparison of data (dots) and atop site theory (solid line) curves. 
Figure 3.1:
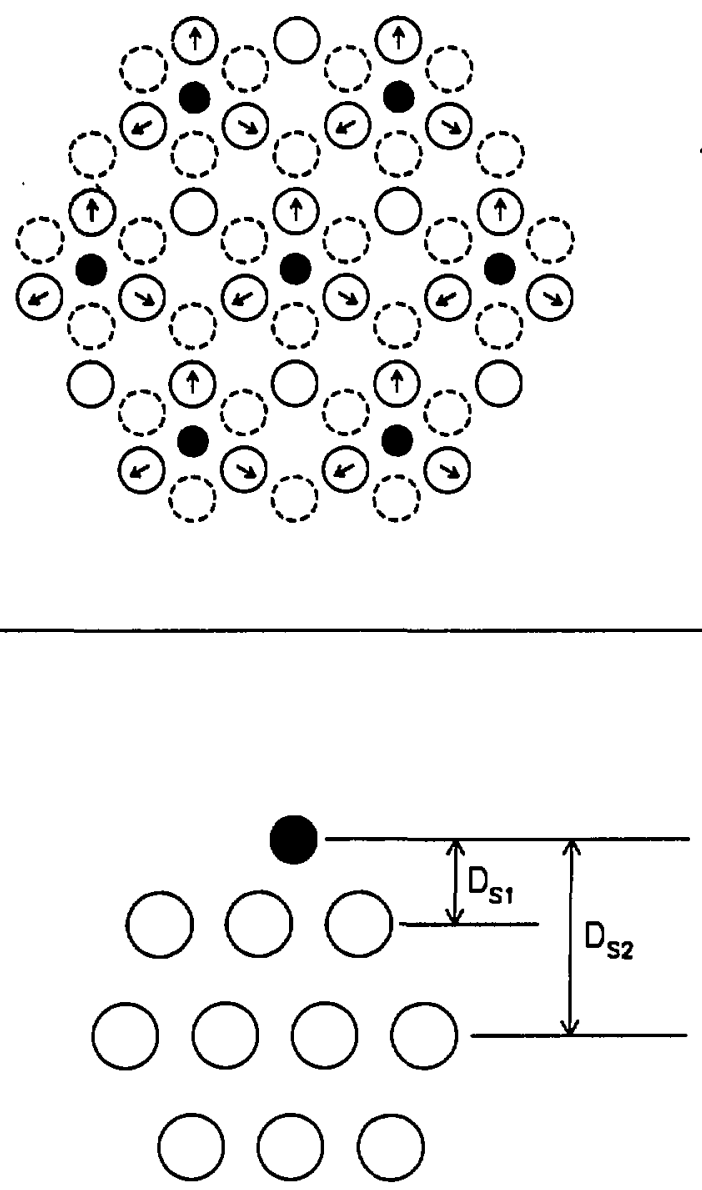
Figure 3.2:

$35^{\circ}$ Off Normal Emission, $195 \mathrm{~K}$

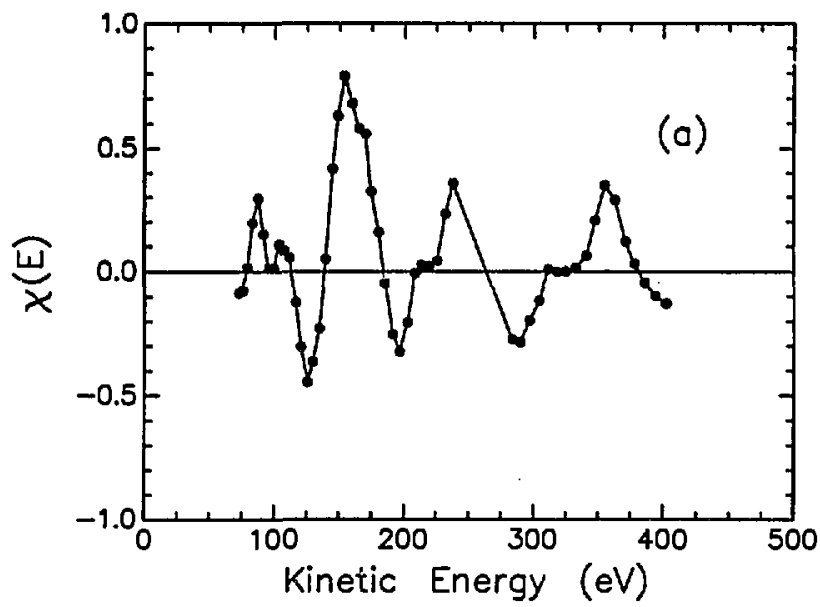

$50^{\circ}$ Off Normal Emission, 195K

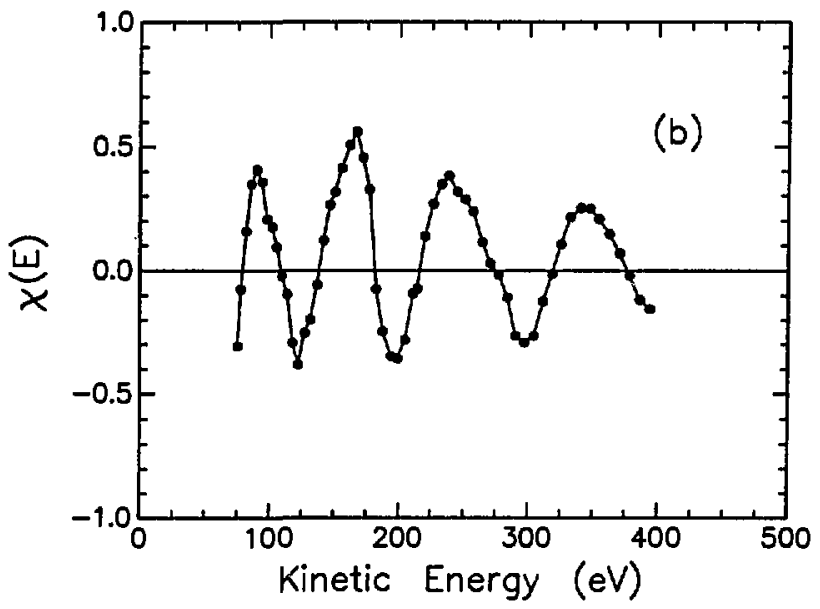


Figure 3.3:

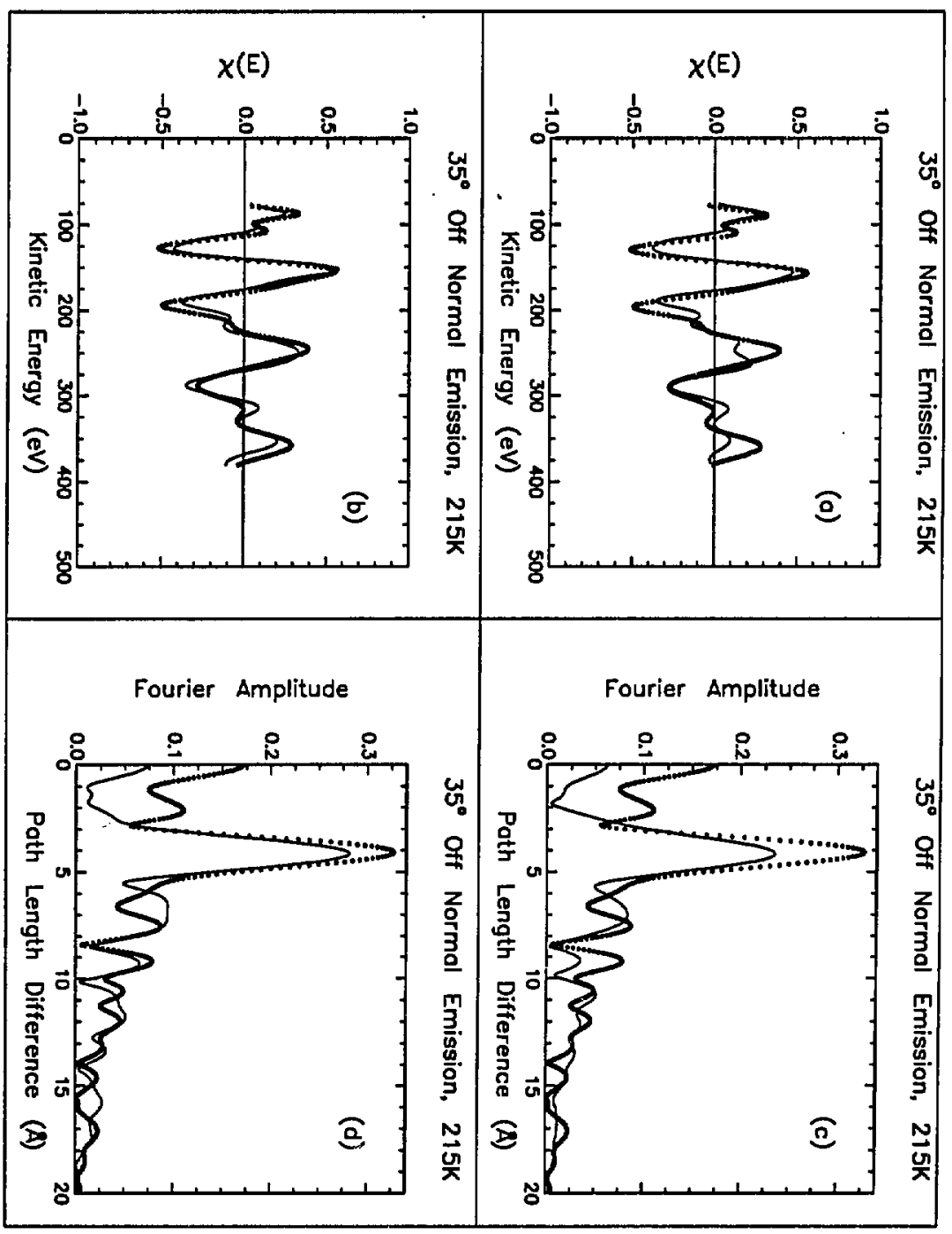


Figure 3.4:

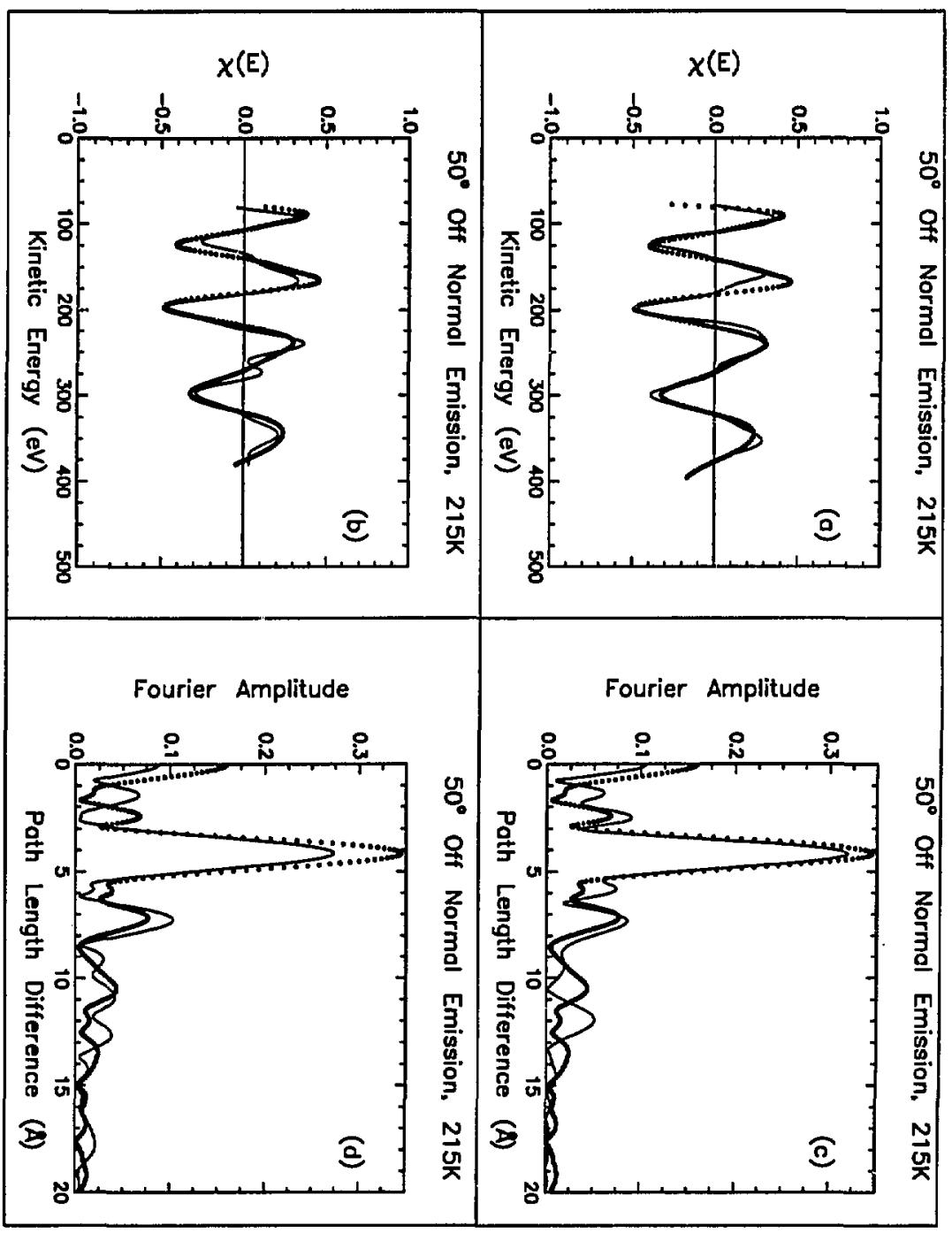


Figure 3.5:

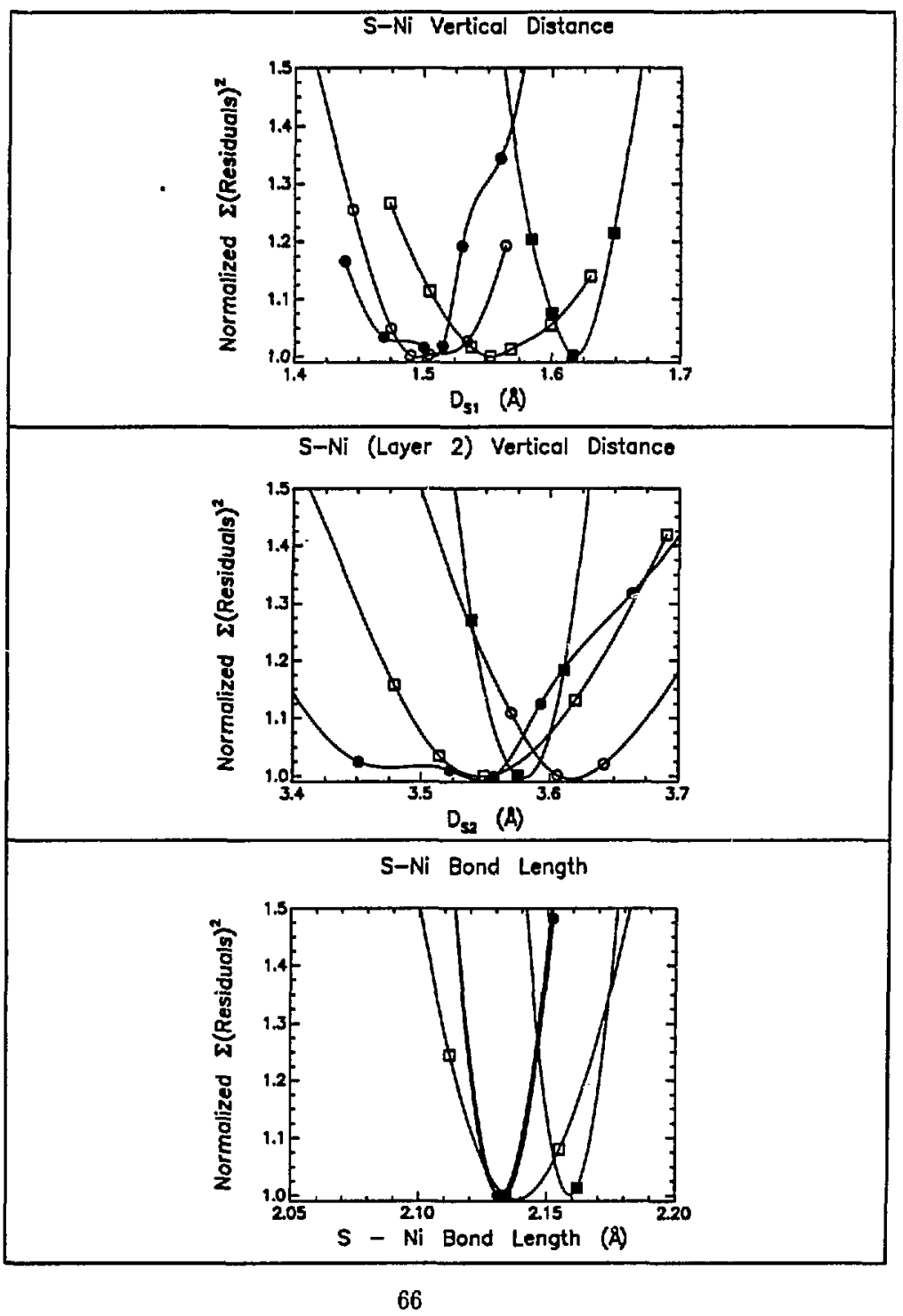




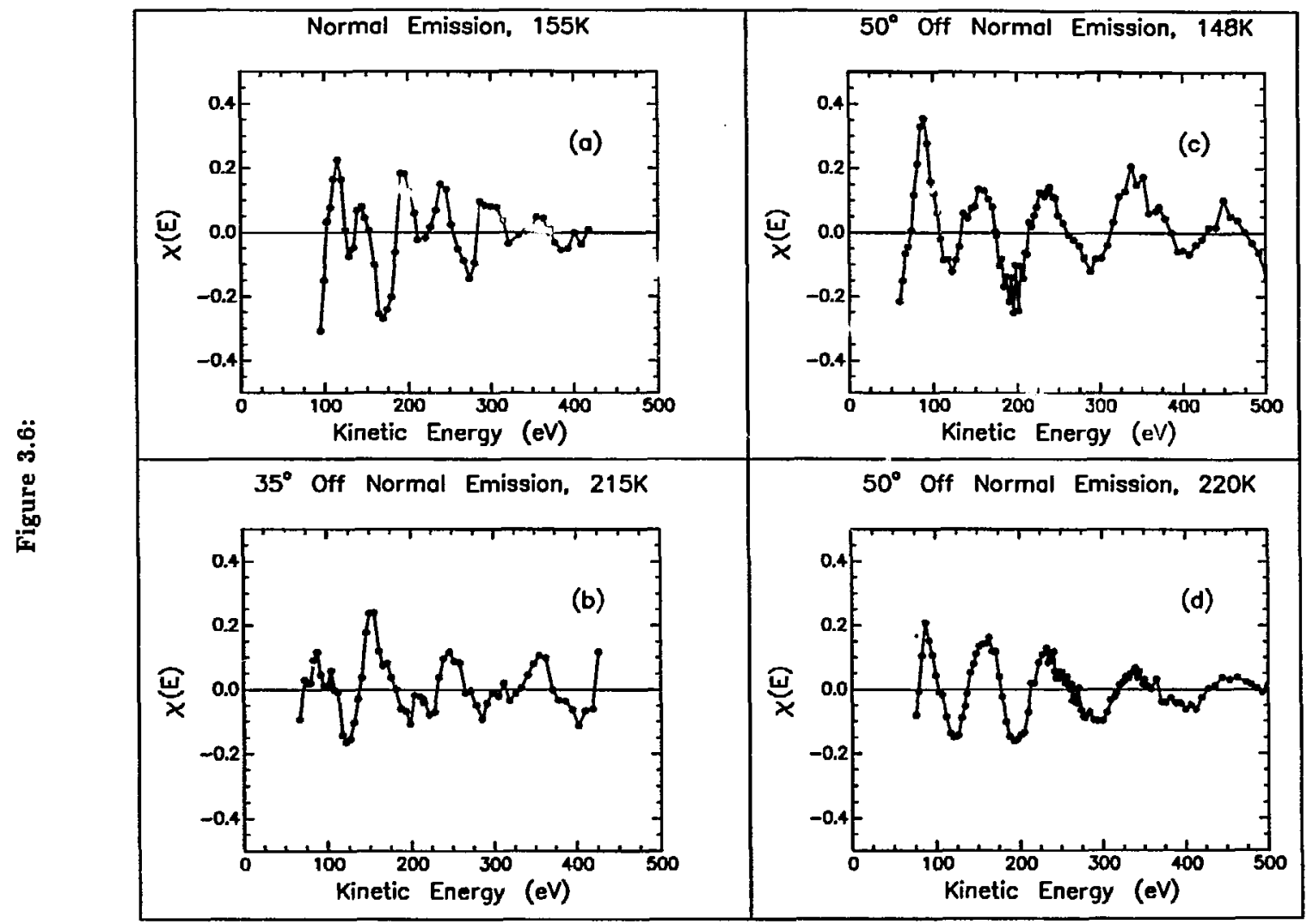


Figure 3.7:

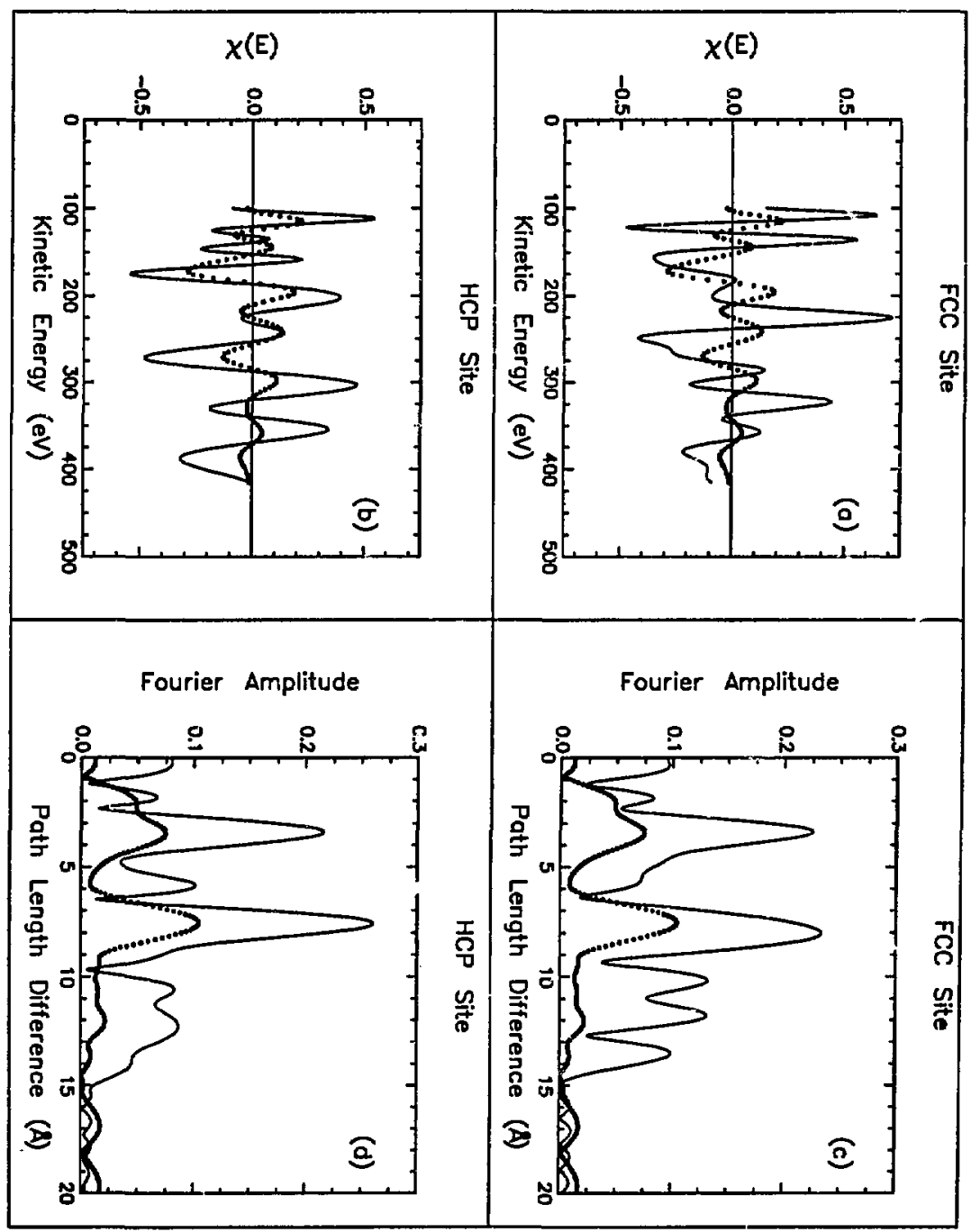


Figure 3.8:

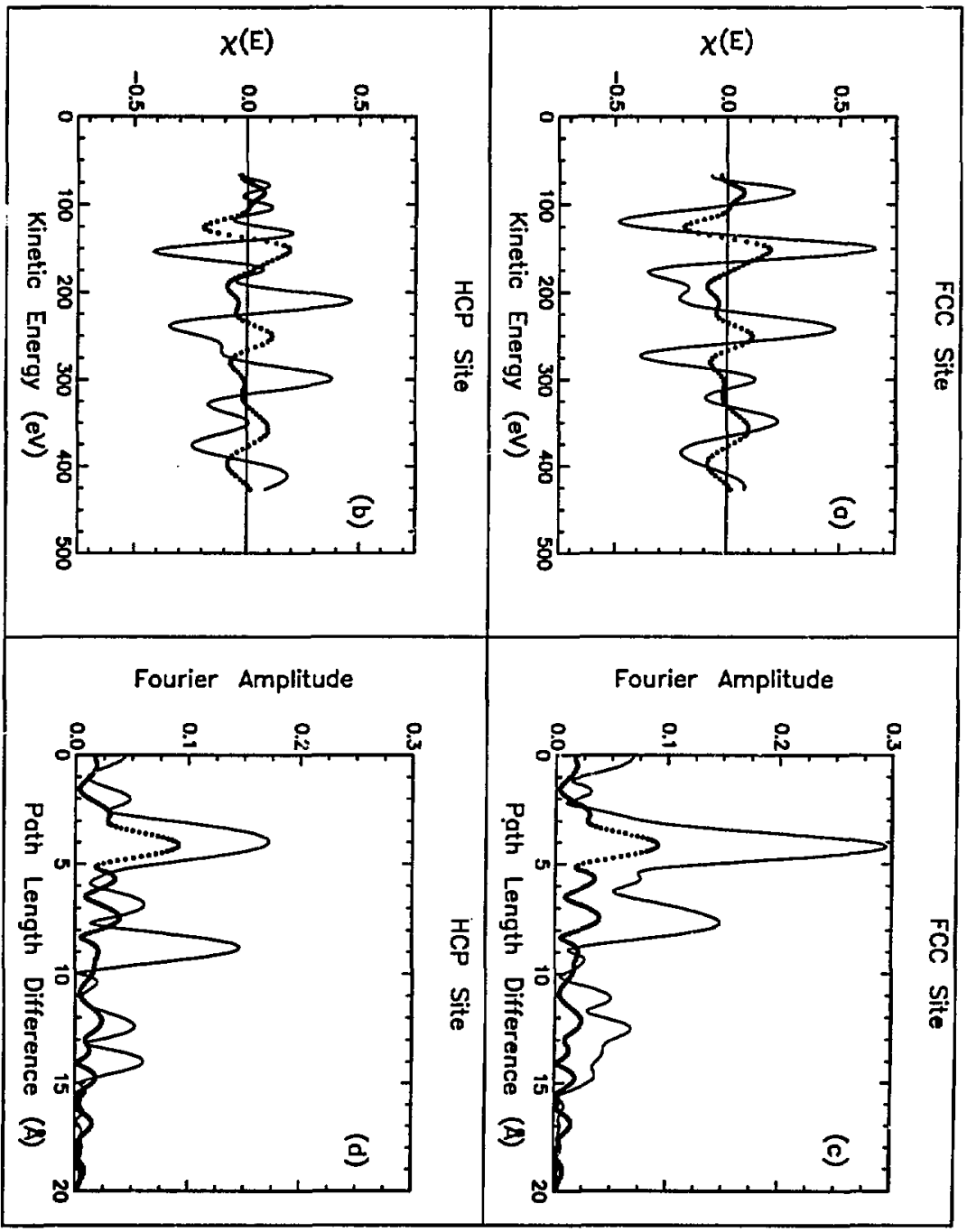


Figure 3.9:

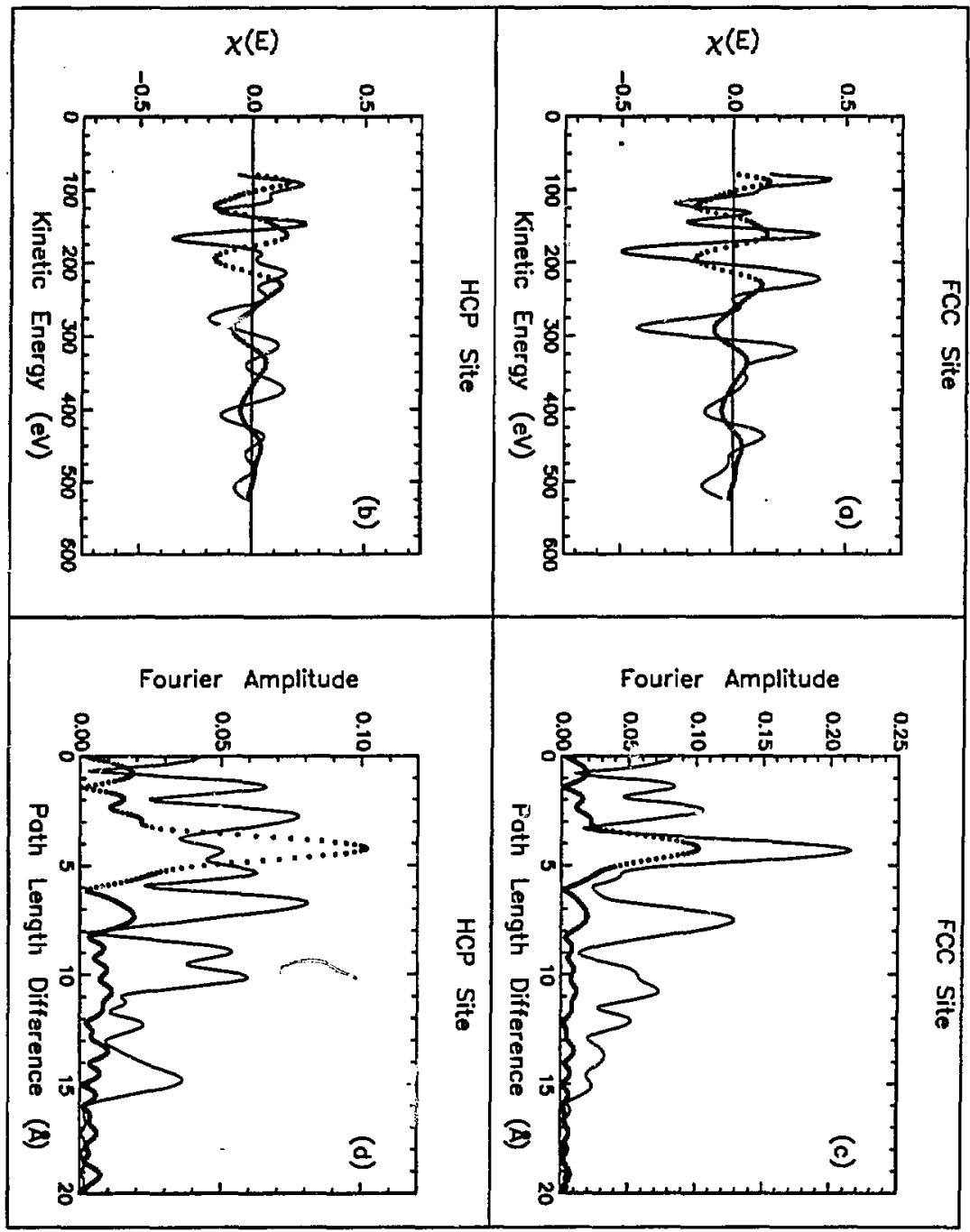


Figure 3.10:

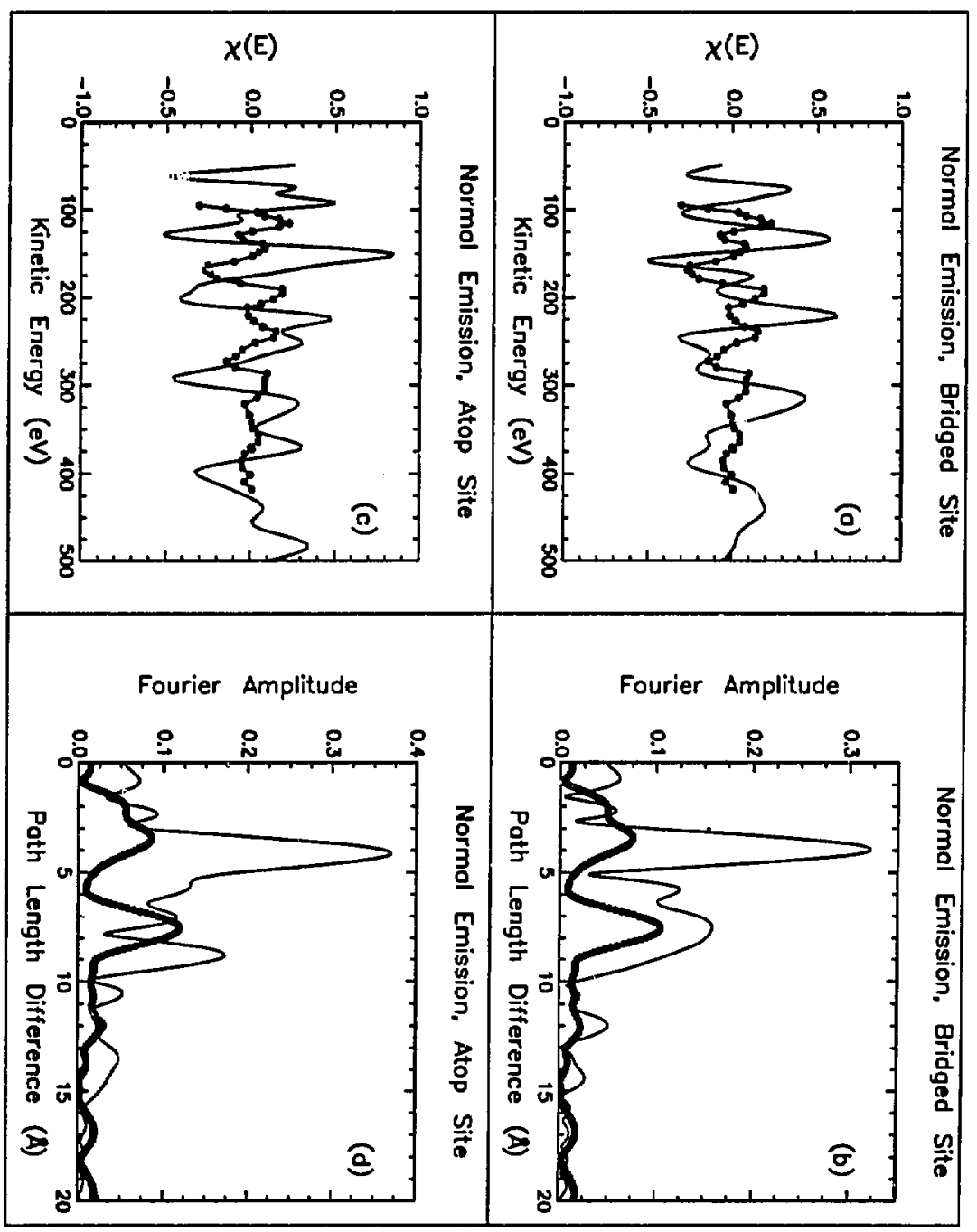


Figure 3.11:

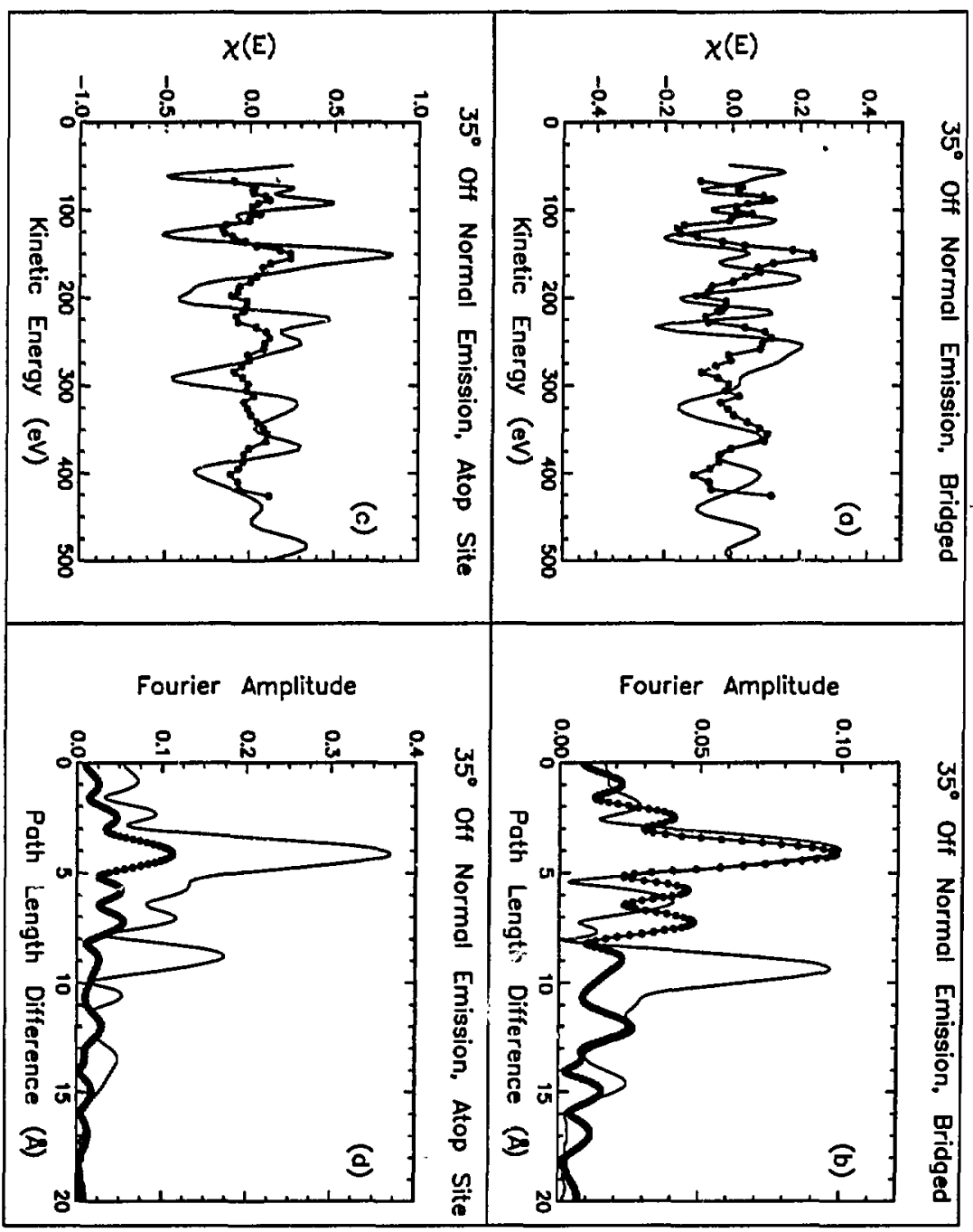


Figure 3.12:

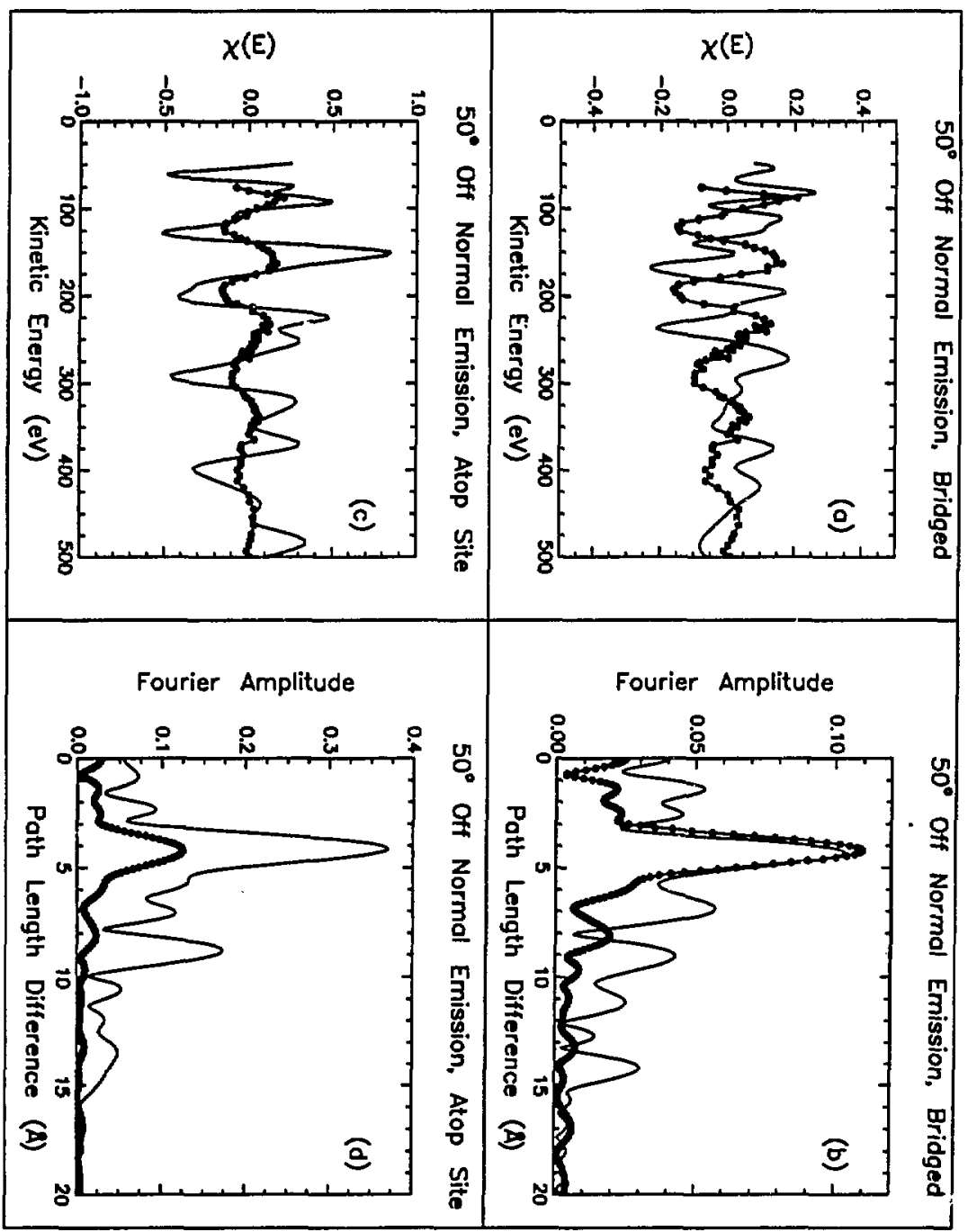




\section{Chapter 4}

\section{ARPEFS as an Analytic Technique}

\subsection{Introduction}

As we have seen in $\mathrm{p}(2 \times 2) \mathrm{S} / \mathrm{Cu}(001)$, ARPEFS is capable of giving structural information with a high degree of accuracy and precision. What is perhaps not as apparent from Chapter 1, however, are the constraints on performing and analyzing an ARPEFS experiment. The purpose of this chapter is to describe in detail the points we have found necessary for an experiment to succeed and to highlight constraints in performing ARPEFS. The steps in an ARPEFS experiment may be outlined:

1. Getting the Data

2. Modelling the Data

\subsection{Getting the Data}

As has been mentioned elsewhere ${ }^{55,56}$, ARPEFS data are collected in two sets. In the first set, $1 \mathrm{~s}$ spectra (in the present case; $p, d, f$, etc. initial states could also be used) are collected in $0.05 \AA^{-1}$ to $0.1 \AA^{-1}$ increments from $k \sim 4.5 \AA^{-1}$ to 
k $11.5 \AA^{-1}$. The second set of spectra are the long 'empirical background' spectra used to normalize the the 1s spectra prior to calculation of the $I(E)$ curve, from which a $\chi(E)$ curve (and using the deBroglie equation, a $\chi(k)$ curve) is extracted.

\subsubsection{1s Spectra}

A sample 1s photoemission spectrum is shown in Figure 4.1, along with the functions used to fit it. As mentioned elsewhere ${ }^{55,56}$, these are a Voigt function, a Gaussian broadened step function used to represent the contribution of inelastic processes to the total photoelectrc intensity, and the (smoothed) empirical background. Each 1s spectrum is fit with five parameters: the empirical background height, the G-step height, and the width, area, and mean of the Voigt function (recall that the G-step mean and width are set to be equal to the Voigt mean and width).

We see that the 1 s photopeak has tailed to zero intensity within a range of $\pm 6 \mathrm{eV}$ about the mean. We have found that for good fitting the 1s spectra should be about twice this width. (In the case ${ }^{57}$ of $\mathrm{c}(2 \times 2) \mathrm{Cl} / \mathrm{Cu}(001)$, where the $\mathrm{Cl}$ is peak is slightly wider, it was necessary to take scans $30 \mathrm{eV}$ wide). We see that $\sim \frac{1}{4}$ of the spectrum is determined solely by the height of the empirical background. Another $\sim \frac{1}{4}$ of the spectrum is determined solely by the height of the empirical background and the G-step. Thus, two of the five fitting parameters are well determined, and have little influence on the remaining fitting in the region of the photopeak. The photopeak parameters of interest are its mean (easy to determine) and its area (at this point a straight exercise in counting). We approximate the photopeak as a Voigt function (a Gaussian to model instrumental resolution convoluted with a Lorentzian to model the intrinsic lineshare). The overall instrumental resolution is treated as an adjustable parameter, and is on the order of $2 \mathrm{eV}$. The 
Lorentzian width is on the order of $1 \mathrm{eV}^{4,55,56}$. The slight $(0.2 \mathrm{eV})$ uncertainty in this last value has not been found to affect significantly the determination of the peak area, which is the parameter we are after. As a side note, we have found that a peak height of at least 2000 counts is desirable for accurate curve fitting. The peak in this spectrum has a peak height of 4000 counts. Thus, we would say that the ideal ls spectrum has an overall width twice the base width of the photopeak and has a peak height of several thousand counts.

\subsubsection{Background Spectra}

Having measured the is spectra the next job is to normalize them so that the intensity modulation may be determined. This is done using the so-called 'empirical background' spectrum. In Figure 4.2a we show unnormalized 1s spectra from the $40^{\circ}$ off normal emission data of $p(2 \times 2) S / C u(001)$, in the kinetic energy range $50 \mathrm{eV}-200 \mathrm{eV}$. In Figure 4.2b we show the rescaled spectra, lined up so that the high kinetic energy sides join smoothly. The envelope of the high kinetic energy sides very closely resembles the inelastic electron distribution shown in Figure 4.3. It is this inelastic electron spectrum that is used to normalize ls spectra in an ARPEFS expericizent. There are two ways to obtain this inelastic tail background. The first would be to take wide ls spectra and line up the high kinetic energy sides. For practical reasons we do not use this approach. We would have to add $5 \mathrm{eV}^{\prime}$ to $10 \mathrm{eV}$ to the high kinetic energy side of each spectrum, so that this high energy tail would extend sufficjently into its neighbor. Given that a typical $\chi(E)$ curve comprises 70 - 100 ls spectra, this would work out to 500 to $1000 \mathrm{eV}$ of extra scanning. With our typical spectrum width of $25 \mathrm{eV}$, this is the equivalent of 20 to 4015 spectra that we would lose (recall that the amount of team time is fixed and is the limiting factor on the number of 1s spectra we are able to take in 
the first place). What we do instead is to measure the inelastic spectrum and use this as a normalization reference.

The empirical background spectrum used to normalize the 1s spectra obviously has to cover the entire range over which the 1s spectra are taken. It also must be slightly wider, since each individual 1s spectrum should extend perhaps $13 \mathrm{eV}$ to either side of the photoemission peak. Thus if one is scar.ning a photopeak kinetic energy range of $60 \mathrm{eV}$ to $560 \mathrm{eV}$ (i.e. $4.3 \AA^{-1}<\mathrm{k}<12 \AA^{-1}$ ), the background spectrum should be at least $530 \mathrm{eV}$ wide. Our analyzer, however, can take a spectrum at most $195 \mathrm{eV}$ wide. Thus this background spectrum must be taken in several partially overlapping segments. We have found a $30 \mathrm{eV}$ overlap to be quite adequate for joining the spectra. Thus, the first segment would cover the range $45 \mathrm{eV}$ to $240 \mathrm{eV}$, the second segment would cover $210 \mathrm{eV}$ to $405 \mathrm{eV}$, and the third segment would cover the range $375 \mathrm{eV}$ to $570 \mathrm{eV}$. The various segments may then be fitted to each other and joined. Since the empirical backgro:nd spectrum is supposed to represent the background of the individual ls spectra, we typically choose the photon energy so that the adsorbate 1s peak is just below (say $15 \mathrm{eV}$ ) the low energy side of the subspertrum. Thus the background will have been taken using a photon energy that is quite close to the photon energy used for the 1s spectrum sitting on that portion of the background. From our experience, the background mesh may be twice as coarse as that used in for the individual photoemission spectra (i.e. $0.6 \mathrm{eV}$ per point for the background and $0.3 \mathrm{eV}$ per point for the individual spectra, using our analyzer). The number of scans for each background subspectrum should be at least as great as the number of sweeps used for each pholoemission spectruin. Thus, our empirical background spectrum takes the same amount of time as scanning 12 1s spectra (590 eV total scanning at $\frac{1}{2}$ the point density, divided by $25 \mathrm{eV}$ ). This buys us 8 to 38 extra $\mathrm{k}$ points at the 
end of the data set and saves us the job of normalizing the high energy tails of 100 spectra.

The background subspectra, since they take a fair amount of time to complete, should be taken towards the end of a fill. There are two reasons for this. The first is that step losses in ring current empirically seem to occur more often at the top of the fill and if the beam is going to be lost, it may as well not be during a long scan. The second reason is that the beam current is usually more stable late in the fill and by this time the beamline optics have long since come into thermal equilibrium. Essentially then, the photon intensity and beam position are not changing during the course of a scan. Since we do not have any means for correcting for such changes it is best to take the spectra when changes are less likely to occur. We implicitly (and not necessarily correctly) assume in this normalization technique that during any one scan there is no change in the photon flux on that portion of the sample that is viewed by the analyzer. A sample (smoothed) background spectrum is shown in Figure 4.3.

\subsection{Discussion}

We have found that this method of collecting and fitting of ARPEFS data has several internal safeguards. Use of the empirical background inherently corrects for beam motion across the sample as the photon energy is stepped. If the number of photons hitting that portion of the sample viewed by the analyzer is changed, both the background and photoemission peak are simultaneously affected. Also, wide 1s photoemission spectra allow for reliable fitting.

In Figure 4.4 we show normal emission I(E) curves derived from the SSRL and NSLS data sets of $\mathrm{p}(2 \times 2) \mathrm{S} / \mathrm{Cu}(001)$. Note the generally good agreement between the two curves, and the slight reduction : $r$ the amplitude of the intensity variations 
of the NSLS curve, which was taken at $155 \mathrm{~K}$. This reproducibility indicates that the experimental details described above yield data that are surprisingly (vide infra) self-correcting and independent of the experimental difficulties encountered during a particular run.

The experimental difficulties are not necessarily negligible, which again we demonstrate using $\mathrm{p}(2 \times 2) \mathrm{S} / \mathrm{Cu}(001)$ ARPEFS data. Because of very poor ring operating conditions and lack of remaining beam time, the $110 \mathrm{~K}$ normal emission curve was taken at SSRL with generally poor statistics. In Figure $4.5 \mathrm{a}$ we show a $1 \mathrm{~s}$ spectrum used in the SSRL normal emission I(E) curve. This particular spectrum is typical of those used in the last third (i.e. $\mathrm{KE}<300 \mathrm{eV}$ ) of this $\mathrm{I}(\mathrm{E})$ curve. Because of this quality of statistics this particular curve was repeated at NSLS, where the ring conditions vere much better (yielding count rates higher by a factor of 10 to 40 ). In Figure $4.5 \mathrm{~b}$ we show the equivalent NSLS spectrum. In Figure 4.4, however, we see that the $I(E)$ curves are quite similar at this energy.

Experimental difficulties are not limited to lack of photons. The X-24A Leamline at NSLS $^{24}$ has its own particular problem: the photon beam moves sideways several millimeters as the photon energy is stepped. This beam motion (several millimeters at a distance of several meters) is due to small and unavoidable alignment errors when the Ge(111) crystals are installed in their holders. At SSRL the monochromator ${ }^{23}$ is designed to permit fine tuning of the crystal positions after monochromator installation and bakeout. At the beginning of a run, then, the experimenter can step the photon energy and tweak the crystal mounts (from outside the vacuum) until there is no apparent beam motion over the desired energy range. The NSLS monochromator, on the other hand, provides no such option. The monochromator crystals are aligned as carefully as possible (on a laboratory bench) in their holders. This assembly is then installed in the beamline and baked 
out. The user then hopes for the best. As mentioned earlier, as the crystals are stepped to vary the photon enirgy, the photon beam moves sideways. As a result, the experimental chamber literally has to be pushed sideways several times over the course of a $\chi(E)$ curve. Note the this process would not affect the relative alignment of the sample and analyzer and so does not affect the experimental geometry. The NSLS monochromator also does not compensate for differential heating of the Ge crystals by the photon beam; this causes a slight vertical drift of the photon beam as the monochromator is stepped.

Our analyzer imposes an important constraint on data aquisition. This is due to the low saturation level of the electron detector in our analyzer. Our analyzer ${ }^{2 i, 22}$ uses a resistive anode as a multichannel position sensitive detector. When a count hits the anode its position is calculated as a means of determining the kinetic energy of the electron that caused the count. If two counts are detected too close together in time, the pair of pulses is rejected. Obviously, at higher count rates the fraction of pulses that are rejected will increase. This has the effect that at high count rates the analyzer response becomes non-linear and the resulting data become distorted. In Figure 4.6 we show this effect. In Figure 4.6a, we show normal emission ARPEFS data of $\mathrm{p}(2 \times 2) \mathrm{S} / \mathrm{Cu}(001)$ taken at NSLS, and in Figure 4.6b, we show normal emission ARPEFS data of $\mathrm{p}(2 \times 2) \mathrm{S} / \mathrm{Cu}(001)$ taken at SSRL, at the same energy range. The spectra shown as circles are the 1s spectra; the empirical ackground spectra, which are used to normalize the is spectra, are shown as crosses. The lines without symbols are the background curves, rescaled to match the high kinetic energy sides of the is spectra. The background and is spectra in Figure 4.6a were taken with a high count rate. Note that because of data distortion the ls spectrum can not be normalized accurately. With the lower count rates obtainable at SSRL (see Figure 4.6b), saturation does not occir at 
this energy and the data can still be fitted. This is the reason that the SSRL data in Figure 4.4 extend to lower kinetic energies; the lower portion of the NSLS data set had to be rejected. Perhaps one advantage of this problem, though, is that the mere ability to fit the spectra may be taken as an assurance of data reliability.

Once the I(E) curve has been obtained, we next have to fit a polynomial $I_{0}$ to it to form the $\chi(E)$ curve. In practice a simple quadratic or cubic polynomial is sufficient. While the two polynomials do yield different $I_{o}$ 's, the difference between the resulting $\chi(\mathrm{E})$ curves is slowly varying and small. We remove all low irequency components by high pass Fourier filtering the data at $2 \AA$ to $3 \AA$, thus eliminating the effects of a particular choice of $i_{0}$. We low pass Fourier filter the data $\chi(E)$ curves to remove random noise (which we typically estimate to be approximately $\pm 2 \%$ ). The upper filtering bound also serves to restrict the cluster size needed to model the $\chi(\mathrm{E})$ curve.

\subsection{Modelling the Data}

We have seen that the methods of ARPEFS data collection and reduction are robust ard self-correcting. Thry yield $\chi(E)$ curyes that are surprisingly unaffected by vagaries of beam stability (as long as the beam is relatively stable during a particular scan) and choice of functional form of the $I_{0}$. This gives us confidence that the methods described above can yield consistent and reliable $\chi(\mathrm{E})$ curves, which we will now try to fit.

\subsubsection{Calculating Theoretical TSMQNE Spectra}

We use the TSMQNE model developed by $\operatorname{Barton}^{25}$. This model includes

1. Spherical Wave Scattering, approximated using the Taylor Series Magnetic Quantum Number Expansion, 
2. Multiple Scattering, through $10^{\text {th }}$ order (maximum),

3. Atomic vibration, using a correlated Debye model,

4. Muffin-tin potentials,

5. analytic treatment of the finite angular acceptance of the analyzer, and

6. damping of wave amplitudes (and thus interference) using an inelastic mean free path.

Item 1, the TSMQNE approximation, has been shown to be an excellent approximation to a full spherical wave treatment ${ }^{58}$ over the $\mathrm{k}$ range $5 \AA^{-1}$ to $12 \AA^{-1}$. Item 2, the multip!e scattering, is selected by amplitude cutoffs as a time-saving device. The program calculates the amplitude of the scattered wave, and if the amplitude is greater than the user-specifier! cutoff, the program proceeds to the next level of scattering. Item 3 , the correlated Debye-Waller treatment, is a bit more complex. In our code we can specify a Debye temperature for the $X, Y$, and $Z$ vibrations of the adsorbate atom, and for the $X, Y$, and $Z$ vibrations of the substratie surface atoms. Exponential decay lengths for the substrate Debye temperatures to the isotropic bulk value are also user-specified. The muffin tin potentials for item 4 are generated by standard techniques, e.g. superposition potentials ${ }^{29}$ or isolated atom potentials ${ }^{69}$. For the latter, electron wavefunctions outside the muffin tin radius are truncated and set to zero. The wavefunctions inside the sphere are either renormalized or an average charge density is added to the muffin tin sphere to preserve charge neutrality The slight variations in the resulting phaseshifts have an effect smaller than the $0.01 \AA$ scatter in our structural determinations. Barton ${ }^{25}$ concluded that even substituting $\mathrm{Cu}$ phaseshifts for $\mathrm{Ni}$ phaseshifts would introduce a $0.02 \AA$ error in path length difference determinations. Item 5 , the inelastic mean 
free path treatment is a simple approximation. The loss of electron wave intensity is assumed to be isotropic anci dependant solely on distance within the solid. The inelastic mean free path $\lambda$ used for the experiments described here $e^{55,56}$ was

$$
\lambda(\AA)=0.75 \mathrm{k}\left(\AA^{-1}\right),
$$

where $\lambda$ is in units of $\AA$ and $k$ is in units of $\AA^{-1}$. This form is quite close to the theoretical relation ${ }^{60}$

$$
\lambda(\AA)=\alpha(\mathrm{E}(\mathrm{eV}))^{\beta},
$$

where for Ju, $\alpha=0.312$ and $\beta=0.5 \mathrm{E}$. for $200 \mathrm{eV}<\mathrm{E}<400 \mathrm{eV}$. For the range $400 \mathrm{eV}<\mathrm{E}<2000 \mathrm{eV}$, these values become 0.133 and 0.695 , respectively. In Figure 4.8 we show these theoretical functions (thin line: Eq. 4.1, thick line: Eq. 4.2) along with experimental values (crosses ${ }^{61}$, $\operatorname{circles}^{62}$, and diamonds (universal curve)). The theoretical expressions do not fit the data very well. To see what effect these other values might have on the fits, we refit the $40^{\circ}$ off normal emission data of $\mathrm{p}(2 \times 2) \mathrm{S} / \mathrm{Cu}(001)$, using the following functional forms of the IMFP:

$$
\begin{aligned}
& \lambda(\AA)=0.75 \mathrm{k}\left(\AA^{-1}\right) \text { (our default form, to check for convergence), and } \\
& \lambda(\AA)=6 \AA \text { (k independent form, approximate fit to Seah's data). }
\end{aligned}
$$

The results are shown in Table 4.1. We see that there has been a $0.001 \AA$ to $0.008 \AA$ change in the structural parameters when the default IMFP was used. This tells us how well our search algorithm had converged. When the 'Seah' form of the IMFP was used, there are shifts in some of the structural parameters, implying that the IMFP is an importani input to the modeling. We point out, though, that the essential conclusions of the $\mathrm{p}(2 \times 2) \mathrm{S} / \mathrm{Cu}(001)$ sttedy (and the basic disagreements between the structure as determined by ARPEFS and other techniques), that $D_{S 1}>1.31 \AA, D_{S 3}-D_{S 1}<3.6 \AA$, and $D_{2 C}-D_{2 A} \sim 0.1 \AA$, remain unchanged. There is a slight increase in the bond length and an increase in the 
$\mathrm{R}$-factor as well. Cost constraints precluded performing the same checks on the other $\mathrm{p}(2 \times 2) \mathrm{S} / \mathrm{Cu}(\mathrm{COO1}) \chi(\mathrm{E})$ curves.

\subsubsection{Varying the Structural Parameters}

This used to be done by hand. In the interests of distancing human influence from the fit, we have switched to computer selection of parameters. Since the actual mechanics have occasioned some interest among former group members, we shall describe them here.

Manual fitting of a $\chi(k)$ curve involves several steps. The first is to decide what particular parameter values one wishes to use in a calculation of a theoretical $\chi(k)$ curve. Next, one writes the command file (a scattering text file, in group parlance). This is then used to run the TSMQNE code to produce a theoretical $\chi(k)$ curve. The theoretical $\chi(k)$ curve is then Fourier filtered in the same way as was the experimental curve, to remove (rather, to make consistent) any artifacts. The theoretical and experimental $\chi(k)$ curves are then compared. New parameters are selected and this process is repeated until the experimenter is satisfied.

This process can, of course, be automated. Optimization routines require that they be given a number (an ERROR or R-factor value, say) for a particular set of parameters. The routine will then vary the parameters in an effort to reduce this number. In FORTRAN this is typically done by minimizing, for example, FUNCTION ERROR(PARRAY), where ERROR is an EXTERNAL function and PARRAY is the particular set of parameter values. The optimization routine will come up with a particular PARRAY, learn the value of ERROR(PARRAY), and choose a nev PARRA $i$.' This process is repeated until user specified convergence criteria are met or until the user decides that the fit is adequate. For our purposes implementing the above process is straightforward. The purpose of the 
EXTERNAL function ERROR is to return an ERROR value for the $\chi(k)$ curve based on the PARRAY structure. So, we have ERROR perform several steps. The first is to write a scattering text file based on the structure described by PARRAY. This is conveniently done by passing PARRAY to a subroutin ' we call it MAKTXT), which consists solely of WRITE and FORMAT statements. This routine writes the tex! file, incorporating the new parameters as it goes along. Once MAKTXT has RETURNed, ERROR performs a CALL LIB\$SPAWN('@DOJT'). This spawns a sub-process to execute the commands contained in DO.IT.COM. The file DOIT.COM causes our new text file to be processed by CHPSCT ( $\mathrm{ur}$ scattering program). DO_T.COM then takes the output file written by CHPS( $T$ and via another command file uses RPN (our group data analysis program) to Fourier filter the theoretical $\chi$ curve and write the filtered result to a data file. DO_IT.COM then EXITS, allowing the parent process to continue. ERROR then READs in the new filtered theoretical curve and calculates the R-factor, which is then passed back to the optimization routine. The optimization routine looks at this ERROR value and decides on a new PARRAY, which it then passes to ERROR. The cycle is repeated until a local (which with luck is also a global) minimum is found. Note that by this repeated use of MAKTXT and CALL LIB\$SPAWN we are able to interface our optimization routine (which we can change to suit our needs) to previously debugged executable files (which we may not wish to modify or be able to modify), without having to recompile or relink the latter. This allows us to concentrate on the problem at hand, without being distracted by keeping track of large amounts of source code. This particular work-around obviously can be applied to other problems. 


\subsubsection{Assorted Constraints}

The main constraints in ARPEFS data analysis are the amount of computer time needed to calculate a $\chi(k)$ curve and the inefficiency of the optimization routine used (the reduction of the raw 1 d data is fairly easy (i.e. fitting $\sim 100$ spectra with a Voigt function) and is easily automated). Consider the Fourier transform of our $40^{\circ}$ off normal $\mathrm{p}(2 \times 2) \mathrm{S} / \mathrm{Cu}(001)$ curve shown in Figure 4.7 . We see that there are several possible path length differences which we may wish to use as cluster size cut-offs when we Fourier filter the data and the theory. We may choose to filter at $<11 \AA,<13 \AA,<15 \AA$, or $<17 \AA$. These cut-offs are chosen to lie between peaks in the Fourier transform, so that as the structure is varied and the peaks shift slightly, we reduce the chance of making a cut-off in the middle of a pank. In Table 4.2 we show the CPU time necessary to calculate a $\chi(\mathrm{k})$ curve using a particular maximum path length difference.

While these numbers for computer time may not seem prohibitive, consider that in doir $;$ a nine parameter fit that several hundred $\chi(k)$ curves may be needed during the optimization process. In their original paper ${ }^{37}$, Nelder and Mead estimate that the number of simplex cycles needed for convergence scales as

$$
\mathrm{N}_{\mathrm{tot}}=3.16(\mathrm{D}+1)^{2.11} \text {. }
$$

where $\mathrm{D}$ is the dimension of the fitting space. While our convergence criteria are not as strict as those given by Nelder and Mead, we still need several sets of simplex cycles to reach a mirimum and then several tens of $\chi(k)$ curves to estimate error bars.

The numbers given in Table 4.2 become more imposing, as shown in Table 4.3. These times are for f.tting one $\chi(k)$ curve. Typically the experimenter will have several $\chi(k)$ curves to fit. Some experimenters may also then wish to what effect 
changing such things as inelastic mean free path, vibrational anisotropy, Debye temperatures, and cluster size have on the derived parameters. Then the whole process may be repeated using the Fourier transforms of the $\chi(\mathrm{k})$ curves. As can be seen, large amounts of computer time (as well as human time watching the fitting to see if it is drifting to an absurd minimum and waiting to see what the final answer is) can be spent doing the fitting. This is not to imply that ARPEFS is prohibitively time consuming, but to demonstrate the need for speeding up the fitting process.

There are several areas that could be attacked. The first is in the optimizing routine. The simplex algorithm used here ${ }^{37,38}$ has been described as one of the less efficient $^{63}$ (albeit one of the more failure-proof ${ }^{36}$ ) in the class of optimization routines that do not make use of gradient information when searching for a minimum. It is conceivable that perhaps.a decrease of $30 \%$ in number of $\chi(k)$ curves needed could be achieved through a better algorithm. The main criterion is, however, that the routine not stop prematurely on nine or so parameter fits to 20 independent points (we had to reject several algorithms on this basis).

Possibly more rewarding approaches would be modifications to the CHPSCT code. The code at present makes no use of mirror plane symmetry. Since scattering geometries are usually chosen to be in one of the crystal planes, there would be a factor of two gain in speed in the calculation. Note that even in the case of normal photoemission the photon polarization vector is off-normal, so at best we have mirror symmetry. This factor of two because of symmetry would be quite useful: looking at Table 4.3, reducing the time by this factor would allow us to fit a $\chi(E)$ curve with our usual cluster size cut-off of $13 \AA-17 \AA$ in less than a CPU day on a Vax6420. Our fits so far indicate that we are usually within a degree or two of the desired crystal plane, so fixing this parameter is not a large approximation. 
In any case, it is much less important the structural parameters. These could be determined quite accurately. For the final fit, of course, the angle could be allowed to float. Another, perhaps more difficult (and not obvious), modification of the CHPSCT code would be to have it provide not only the $x(\mathrm{k})$ curve as a function of the input parameters but also provide derivatives with respect to these parameters as well, although to be precise these should be the gradients of the Fourier-filtered $\chi(k)$ curve. Being able to use gradient-dependent optimization routines could conceivably decrease the number of $\chi(k)$ computations by an order of magnitude.

Possibly better solutions involve looking at the scattering processes more carefully. In Figures 4.9 and 4.10 we show plots of the scattering amplitudes of atoms for the $\mathrm{p}(2 \times 2) \mathrm{S} / \mathrm{Cu}(001)$ normal emission and $40^{\circ}$ off normal ARPEFS. In these plots the radius of the atom is proportional to that atom's contribution to the ARPEFS, as determined by the log file printed by the CHPSCT program as it calculates a theoretical $\chi(k)$ curve. Each Figure shows an overall view of the adsorbate system, followed by layer-by-layer plots. In both Figures $4.9 \mathrm{a}$ and $4.10 \mathrm{a}$ we see that the scattering atoms are quite close to the emitter, indeed, they tend to lie in one quadrant. For the off-normal directions we see further that in each layer usually one atom is dominant. (It is this dominance that allows us to assign peaks in the Fourier transforms to individual atoms. It is also the basis for the view that ARPEFS allows us to spotlight particular atoms by suitable choice of experimental geometry.) For the normal emission ARPEFS, we see that atoms are highlighted by the emission direction and the electric field vector of the incoming photons (in Figure $4.9 \mathrm{~d}$, note the approximately equal scattering strengths of the so-called ' $\mathrm{C}$ ' and ' $A$ ' atoms). The essential feature is that most of the ARPEFS signal is created by very few atoms. It has been suggested that one possible approach to speed up 
the fitting of $\chi(k)$ curves would be to restrict consideration of scattering atoms to those lying in a narrow cone around the emission direction. We think that this is a misleading approach. First, the cone is not that narrow. Looking at Figure 4.9d, we see that this cone would be at least $40^{\circ}$ wide (haif-angle) to get known important scatterers, so that there is a small time-saving at best. A better approach would ve to redefine the problem. There are two reasons to calculate a $\chi(k)$ curve: (a) to see the curve for a particular structure and (b) to fit a data $\chi(k)$ curve. In Table 4.2 we see that the time to calculate one curve is about $\frac{1}{3}$ CPU hour on a modern workstation. In practice this is not a serious constraint, since for a given adsorbate system the approximate structure is already known or can be guessed at after a few trial curves. The other reason to calculate a $\chi(k)$ curve is in fitting data. Here, we already approximately know the structure, we are just tweaking the atomic positions. After we have calculated the first $\chi(k)$ curve, we know from the log file which scattering events are important. Shifting atom slightly from their initial positions is unlikely to drastically affect the relative importance of various scattering chains. Thus, the r.sults of the first $\chi(k)$ curve could be used as a good guide for the calculation of the next 299 curves. This approach would eliminate the need for empirical cut-offs and the danger that the cut-off might accidentally

eliminate an atom from consideration as its position is changed. Looking at the log file would also allow us to determine which events are symmetry related, thus eliminating the need to perform redundant calculations.

\subsection{Conclusions}

In summary, the following changes should be made:

Geometry Control At present we can set our experimental geometries to within $2^{\circ}$ of the desired angles. For $40^{\circ}$ off normal $\mathrm{p}(2 \times 2) \mathrm{S} / \mathrm{Cu}(001)$ data, a $2^{\circ}$ er- 
ror in the electron take off angle, in the single scattering approximation, would give a $0.01 \AA$ error in the S-Cu bond length. While we can allow these angles to float in the fitting process, the increase in the number of fitting parameters increases our n̂tting time by $30 \%$ to $60 \%$, assuming Equation 4.3 is valid for our case. It is also inelegant to leave the experimental conditions as adjustable parameters during the fitting. One possible way to solve this might be to use a display type analyzer, so that the geometry could be verified directly before the experiment is started. This may also be a factor in our ability to determine precisely $\mathrm{D}_{\mathrm{S} 1}$ in our $\mathrm{p}(2 \times 2) \mathrm{S} / \mathrm{Cu}(001)$ and $\mathrm{p}(2 \times 2) \mathrm{S} / \mathrm{Ni}(111)$ studies. In both of these systems there is thought to be a horizontal reconstruction of the first layer substrate atoms. In other words, there are two structural parameters that go into determining the path length difference for scattering from these atoms. There are also the two experimental angles that are needed to fix the path length difference (i.e. the emission direction). This appears to overtax our fitting procedures. We saw that for $\mathrm{p}(2 \times 2) \mathrm{S} / \mathrm{Cu}(001), \mathrm{D}_{\mathrm{S} 1}$ was less precisely determined than some of the other structural parameters, where there was only one structural degree of freedom.

Data Aquisition A faster digitizer is required. The present detector saturates at too low a count rate and causes a loss of data which can only be determined after the run is over.

Fitting the $\chi(E)$ curves The CHPSCT code should be modified to take into account mirror symmetry. This alone would speed up the fitting process by a factor of two. Effort should also be made to find an algorithm requiring fewer functions call to minimize our R-factor. 
Temperature Control While we are able to maintain stable $( \pm 2 \mathrm{~K})$ temperatures during a $\chi(E)$ curve, we are not able to select that temperature in advance. The present set-up allows us to run either at room temperature (i.e no $\mathrm{LN}_{2}$ ), 'cold' (i.e. $\left.110 \mathrm{~K}-150 \mathrm{~K}\right)$ with $\mathrm{LN}_{2}$, or intermediate $\left(220 \mathrm{~K}, \mathrm{LN}_{2}\right.$ on, along with the sample heater on low power). While this is adequate for structural studies (where the desire is to freeze out all vibration) on materials with high Debye temperatures, the ability to go to lower experimental temperatures would improve the signal-to-noise ratio of our $\chi(k)$ curves. Our present inability to select experimental temperatures in advance precludes doing temperature-dependent ARPEFS. 


\subsection{Tables}

Table 1.1: Results of $k$-space Fitting $40^{\circ}$ off normal ARPEFS data, $\mathrm{p}(2 \times 2) \mathrm{S} / \mathrm{Cu}(001)$. The fit was between $4.7 \AA^{-1}<\mathrm{k}<11.8 \AA^{-1}$ and $1 \AA<\mathrm{R}<17 \AA$ (i.e., $74 \mathrm{eV}<\mathrm{E}<523 \mathrm{eV}$ ).

\begin{tabular}{|c|c|c|c|}
\hline Parameter & Starting Values & Default IMFP & Seah form \\
\hline $\mathrm{D}_{\mathrm{S1}}$ & 1.342 & 1.339 & 1.346 \\
\hline $\mathrm{D}_{\mathrm{sc}}$ & 3.161 & 3.162 & 3.137 \\
\hline $\mathrm{D}_{\text {so }}$ & 3.179 & 3.184 & 3.119 \\
\hline $\mathbf{D}_{\mathrm{SA}}$ & 3.092 & 3.090 & 3.055 \\
\hline $\mathrm{D}_{\mathrm{S} 3}$ & 4.911 & 4.910 & 4.960 \\
\hline Bond & 2.266 & 2.266 & 2.274 \\
\hline$\Delta$ & +0.023 & +0.025 & +0.030 \\
\hline$\theta_{e}$ & 41.4 & 41.5 & 38.4 \\
\hline$\Delta \phi_{e}$ & 1.5 & 1.3 & 0.2 \\
\hline Start R-factor & .0067 & .0067 & .0124 \\
\hline Final R-factor & .0067 & .0065 & .0072 \\
\hline
\end{tabular}

Table 4.2: CPU time needed to calculate a $\chi(\mathrm{k})$ curve using a particular Path Length Difference Cutoff. This is for a $40^{\circ}$ off normal curve at $140 \mathrm{~K}$. The $\mathrm{Cu}$ atoms are assumed to be in the bulk positions. The curve is calculated for $4 \AA^{-1}<\mathrm{k}<12 \AA^{-1}$ on a 128 point mesh. The times shown are approximate and will vary sligkttly between machines at different sites.

\begin{tabular}{ccccc}
\hline Maximum & \multicolumn{4}{c}{ CPU Time (min) } \\
Path Length & VaxStation & VaxStation & Vax & Vax \\
Difference $(\AA)$ & 2000 & 3100 & 8700 & 6420 \\
\hline 11.3 & 45 & 12 & 5 & 4 \\
13.1 & 58 & 15 & 6 & 5 \\
15.5 & 84 & 22 & 9 & 7 \\
17.5 & 92 & 24 & 9 & 8 \\
\hline
\end{tabular}


Table 4.3: CPU time needed to calculate $300 \chi(\mathrm{k})$ curves using a particular Path Length Difference Cutoff. This is for a $40^{\circ}$ off normal curve at $140 \mathrm{~K}$. The $\mathrm{Cu}$ atoms are assumed to be in the bulk positions. The curve is calculated for $4 \AA^{-1}<\mathrm{k}<12 \AA^{-1}$ on a 128 point mesh. The times shown are approximate and will vary slightly between machines at different sites.

\begin{tabular}{ccccc}
\hline Maximum & \multicolumn{4}{c}{ CPU Time (hours) } \\
Path Length & VaxStation & VaxStation & Vax & Vax \\
Difference $(\AA)$ & 2000 & 3100 & 8700 & 6420 \\
\hline 11.3 & 230 & 59 & 23 & 19 \\
13.1 & 290 & 75 & 29 & 24 \\
15.5 & 420 & 110 & 43 & 35 \\
17.5 & 460 & 120 & 47 & 38 \\
\hline
\end{tabular}




\subsection{Figure Captions}

Figure 4.1: Generic ls photoemission spectrum, along with fitting functions: a Voigt to model the line-shape, a Gaussian broadened step function to model inelastic scattering processes, and the empirical background spectrum.

Figure 4.2: Normalization of individual ls spectra in order to make an $\mathrm{I}(\mathrm{E})$ curve. In (a) we show the individual is spectra as they are taken, that is, with no correction for photon flux and beam position changes. In (b) we show the same spectra after renormal:zation. These spectra are a subset of the $40^{\circ}$ off normal ARPEFS 1s spectra of $\mathrm{p}(2 \times 2) \mathrm{S} / \mathrm{Cu}(001)$.

Figure 4.3: Background spectrum of $140 \mathrm{~K} 40^{\circ}$ off normal data set. This is a composite of three spectra:

1) $50 \mathrm{eV}<\mathrm{KE}<244 \mathrm{eV}, \mathrm{h} \nu=2515 \mathrm{eV}$,

2) $200 \mathrm{eV}<\mathrm{KE}<394 \mathrm{eV}, \mathrm{h} \nu:-2665 \mathrm{eV}$, and

3) $350 \mathrm{eV}<\mathrm{KE}<544 \mathrm{eV}, \mathrm{h} \nu=2815 \mathrm{eV}$.

In each case the photon energy was chosen so that the sulfur 1s photopeak would lie $10 \mathrm{eV}^{\prime}$ below the low kinetic energy end of each subspectrum.

a) Composite spectrum.

b) Smoothed derivative of spectrum shown in (a), showing similarity to Auger spectra taken in derivative mode, and highlighting a sulfur LMM Auger peak at $143 \mathrm{eV}$. and a $\mathrm{Cu} \mathrm{MNN}$ Auger peak at $\sim 59 \mathrm{eV}$.

Figure 4.4: Comparison of the raw I(E) curves obtained at SSRL and NSLS. The reproducibility is generally good. The differences between the spectra are a reduction of amplitude variation in the $155 \mathrm{~K}$ curve compared to the $110 \mathrm{~K}$ curve and a change in the shoulder at $100 \mathrm{eV}$. 
Figure 4.5: Difference in statistics for 1 s spectra used for $\mathrm{I}(\mathrm{E})$ curyes. Despite the lower quality of the SSRL spectrum the $\mathrm{I}(\mathrm{E})$ curves in Figure 4.4 are similar.
(a) SSRL spectrum (symbol $O$ ).
(b) NSLS spectrum (symbol $x$ ).

Figure 4.6: Effect of high count rate on data fidelity. In each frame we show the 1s spectrum $(O)$, the background function $(x)$, and the background function rescaled as it would be for fitting the 1 spectrum (no symbol). For purposes of comparison the data have been rescaled to counts per scan at 1 second per channel.

(a) NSLS normal emission data. Note that the empirical background can not be used for data normalization because of distortion in its curvature.

(b) SSRL normal emission data. The background is not affected by saturation and may be used for data normalization.

Figure 4.7: Fourier transform of $40^{\circ}$ off normal data. In fitting this data pathlength difference cutoffs of $11.3 \AA, 13.1 \AA$, or $15.5 \AA$ might be chosen. The dashed line indicates the assumed $2 \%$ noise level. Note that random noise limits our ability to obtain structural information beyond $16 \AA$. 
Figure 4.8: Different forms of the inelastic mean free path in $\mathrm{Cu}$. For the empirical curves the lines are to guide the eye.

\section{Theoretical Relations:}

Thick line: Theoretical relation $\left(\lambda(\AA)=\alpha E(\mathrm{eV})^{\beta}\right)$ from reference 60 .

Thin line: Default relation $\left(\lambda(\AA)=0.75 \mathrm{k}\left(\AA^{-1}\right)\right)$ used in previous ARPEFS work.

\section{Empirical Relations:}

$x$ : Data from reference 61 .

O: Data from reference 62.

๑: Digitized so-called 'Universal Curve'.

Figure 4.9: Importance of scattering atoms to the ARPEFS, normal emission $\mathrm{p}(2 \times 2) \mathrm{S} / \mathrm{Cu}(001)$. The radius of the atoms is proportional to their contribution to the ARPEFS, as determined from the CHPSCT log files. Recall that the adsorbate unit cell for $\mathrm{p}(2 \times 2) \mathrm{S} / \mathrm{Cu}(001)$ is $5.10 \AA$ on a side, thus the $\mathrm{S}$ adatoms sit approximately on the intersections of the grid lines. The photon polarization is in the [111] direction, thereby highlighting substrate atoms in the third quadrant.

a) Overview, showing all layers.

b) Plot showing scattering importance of the adsorbate $\mathrm{S}$ atoms.

c) Plot showing scattering importance of the $\mathrm{Cu}$ atoms in the first layer.

d) Plot showing scattering importance of the $\mathrm{Cu}$ atoms in the second layer. Note that the ' $\mathrm{C}$ ' and ' $\mathrm{A}$ ' atoms are of approximately equal strength, and that the ' $O$ ' atom is ignored.

e) Plot showing scattering importance of the $\mathrm{Cu}$ atoms in the third layer.

f) Plot showing scattering importance of the $\mathrm{Cu}$ atoms in the fourth layer. 
Figure 4.10: Importance of scattering atoms to the ARPEFS, $40^{\circ}$ off normal emission $\mathrm{p}(2 \times 2) \mathrm{S} / \mathrm{Cu}(001)$. The radius of the atoms is proportional to their contribution to the ARPEFS, as determined from the CHPSCT log files. Recall that the adsorbate unit cell for $\mathrm{p}(2 \times 2) \mathrm{S} / \mathrm{Cu}(001)$ is $5.10 \AA$ on a side, thus the $S$ adatoms sit approximately on the intersections of the grid lines. The photon polarization is in the [111] direction, thereby highligiting substrate atoms in the third quadrant.

a) Overview, showing all layers.

b) Plot showing scattering importance of the adsorbate $\mathbf{S}$ atoms. The central atom is probed by serving as a forward scatterer for the backscattering events of the deeper $\mathrm{Cu}$ layers.

c) Plot showing scattering importance of the $\mathrm{Cu}$ atoms in the first layer. Note the dominance of the backscattering atom and the relative unimportance of the other atoms in this layer.

d) Plot showing scattering importance of the $\mathrm{Cu}$ atoms in the second layer. Note the strength of the ' $\mathrm{A}$ ' atom and the relative unimportance of the ' $\mathrm{C}$ ' and ' $O$ ' atoms.

e) Plot showing scattering importance of the $\mathrm{Cu}$ atoms in the third layer. Again, only the backscattering atom is of interest.

f) Plot showing scattering importance of the $\mathrm{Cu}$ atoms in the fourth layer. 
Figure 4.1:

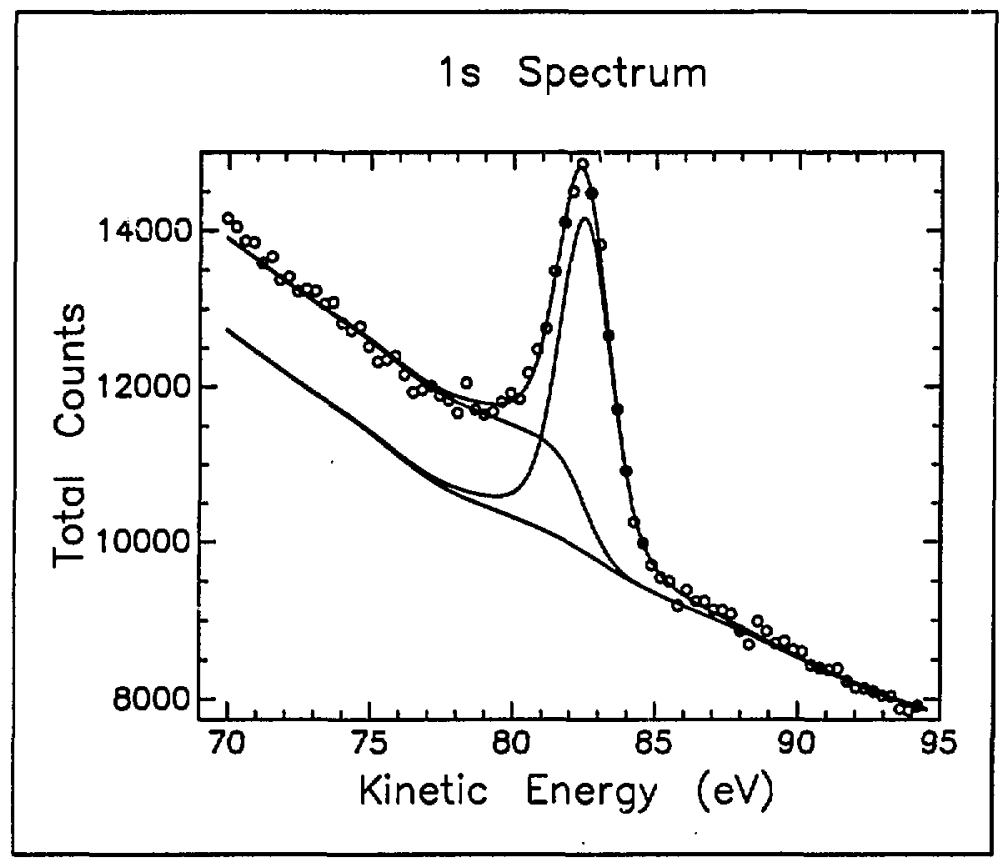


Figure 4.2:

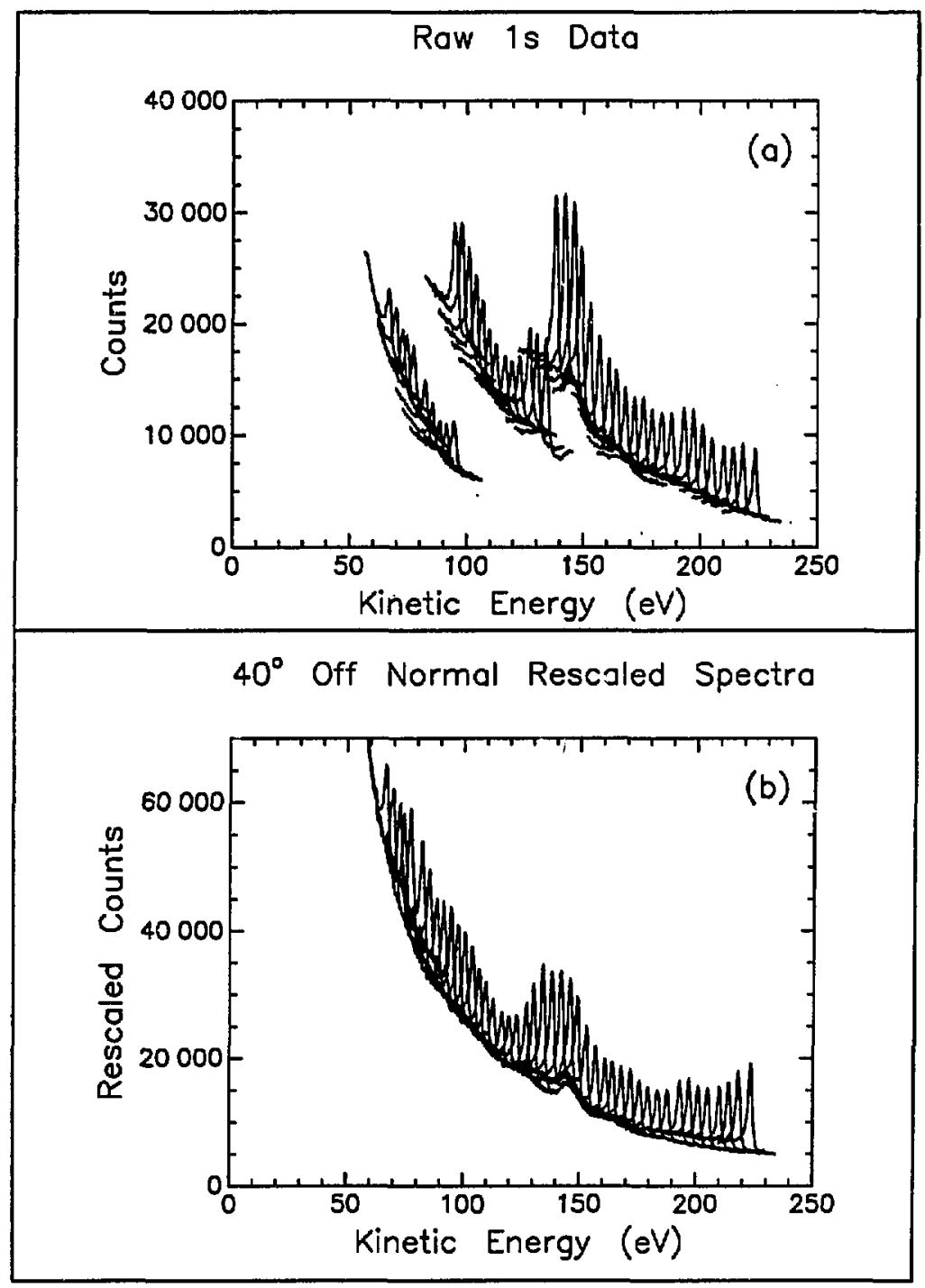


Figure 4.3:

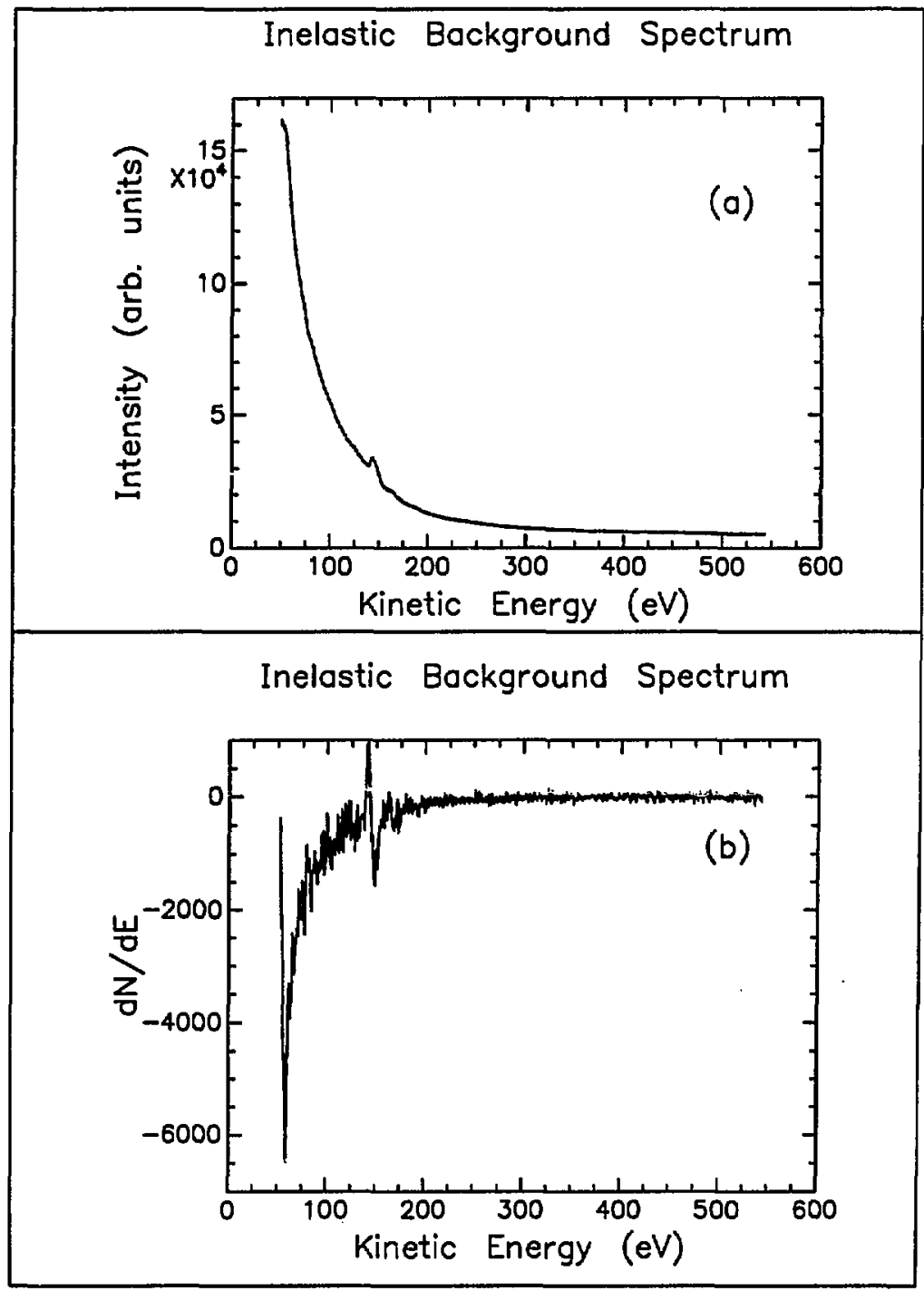


Figure 4.4:

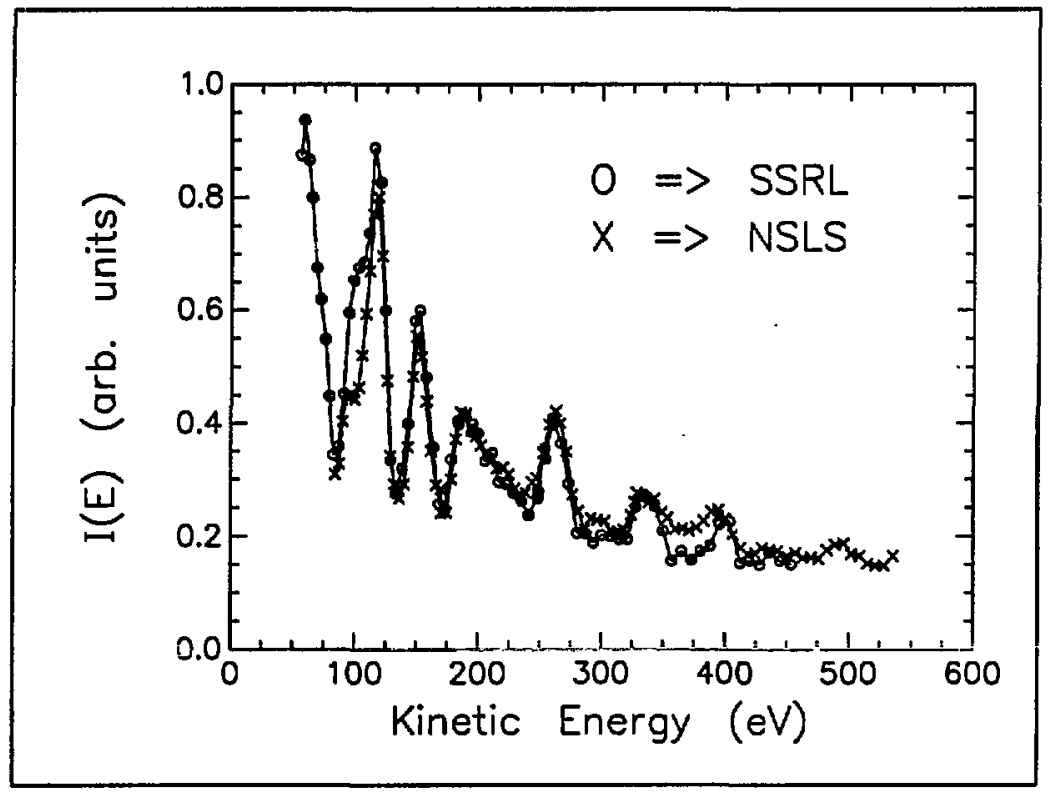


Figure 4.5:

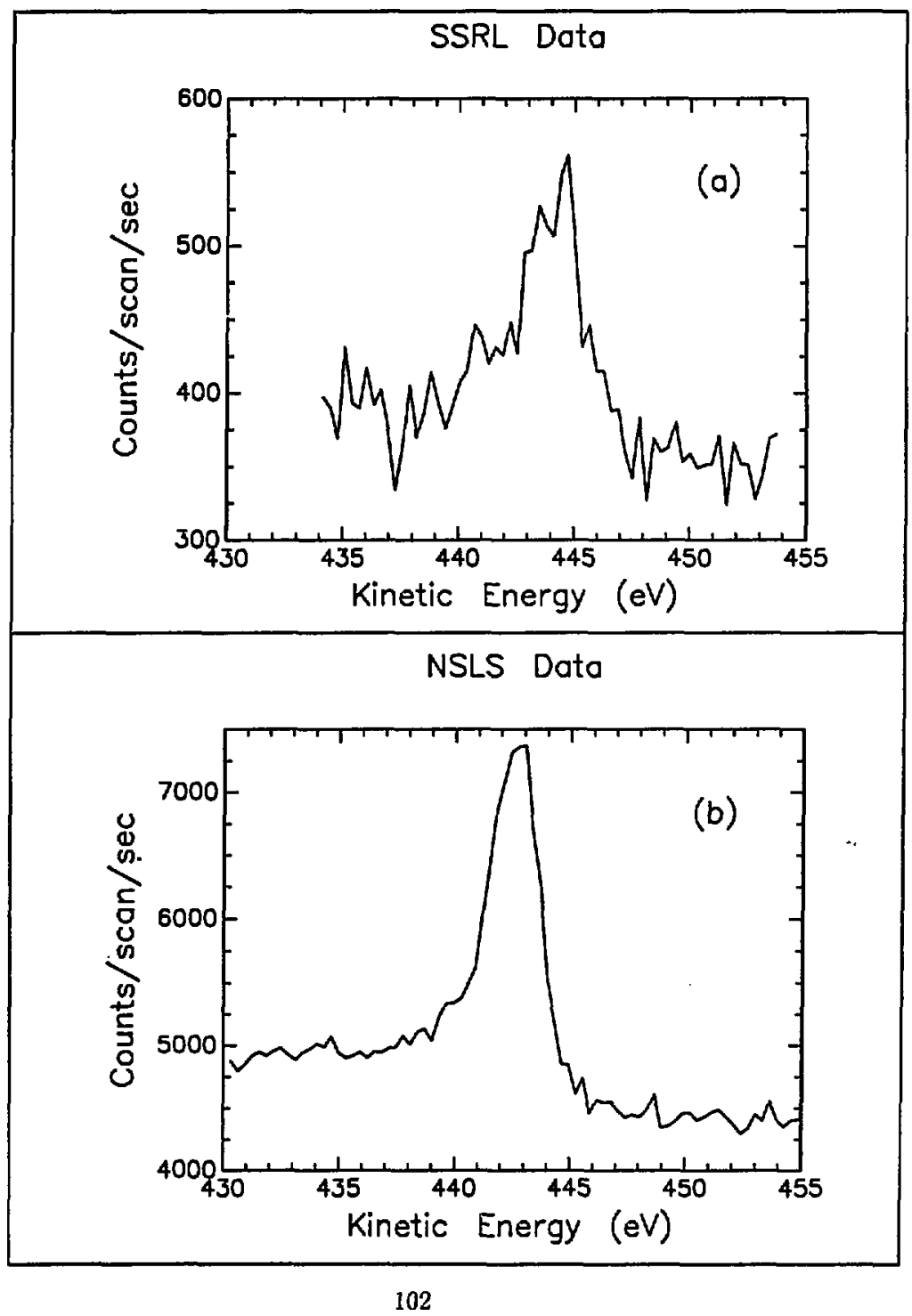


Figure 4.6:

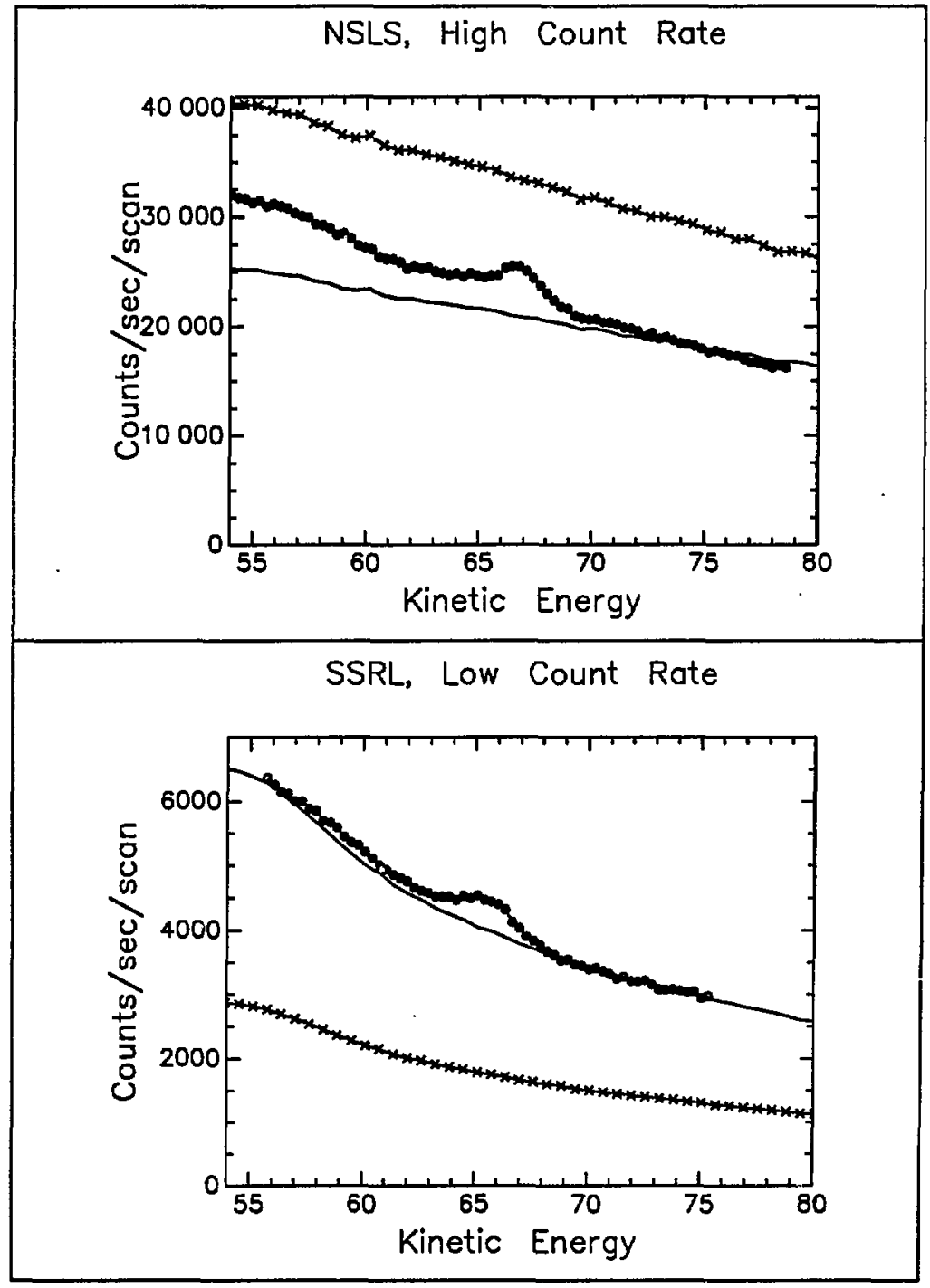


Figure 4.7:

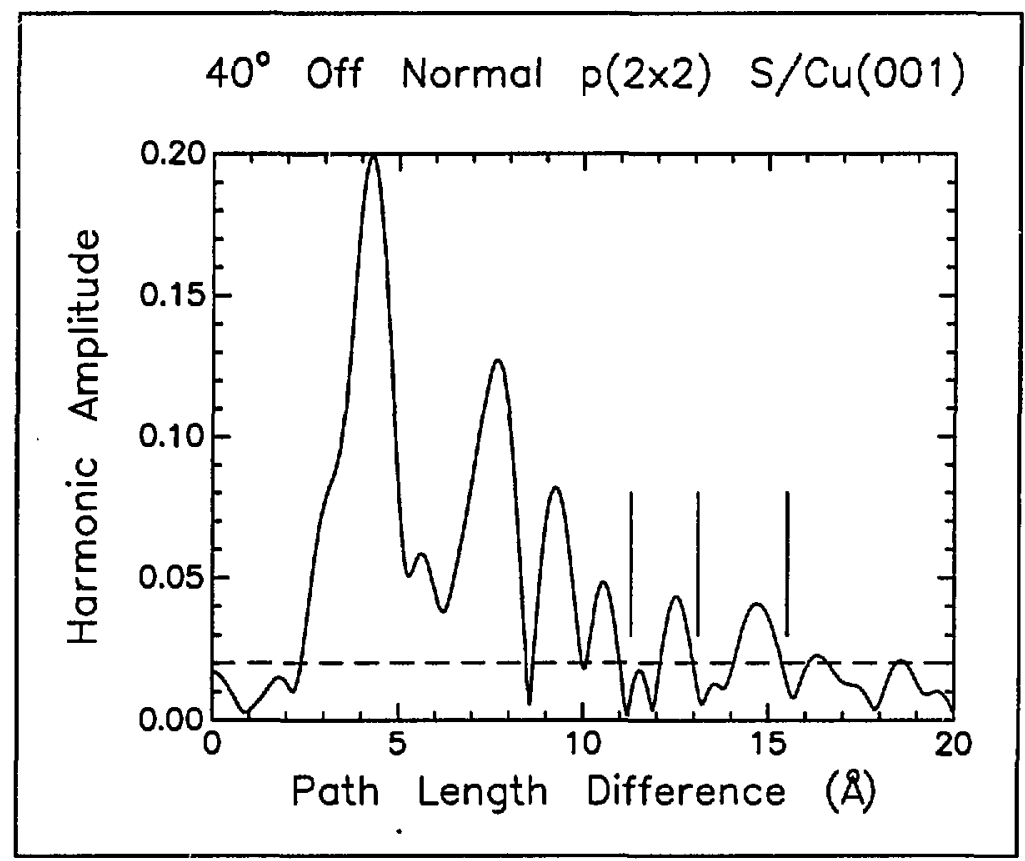




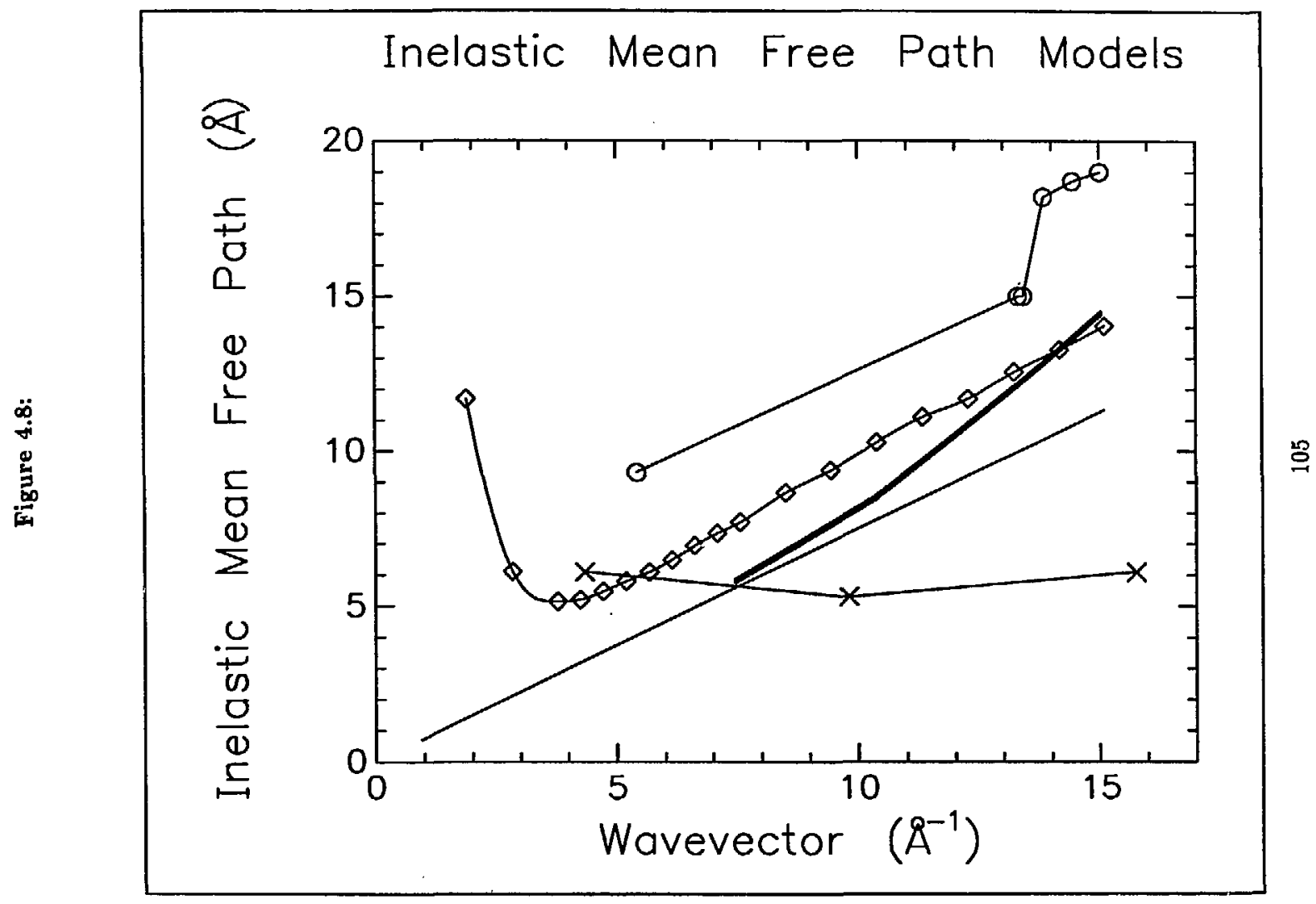


Figure 4.9:

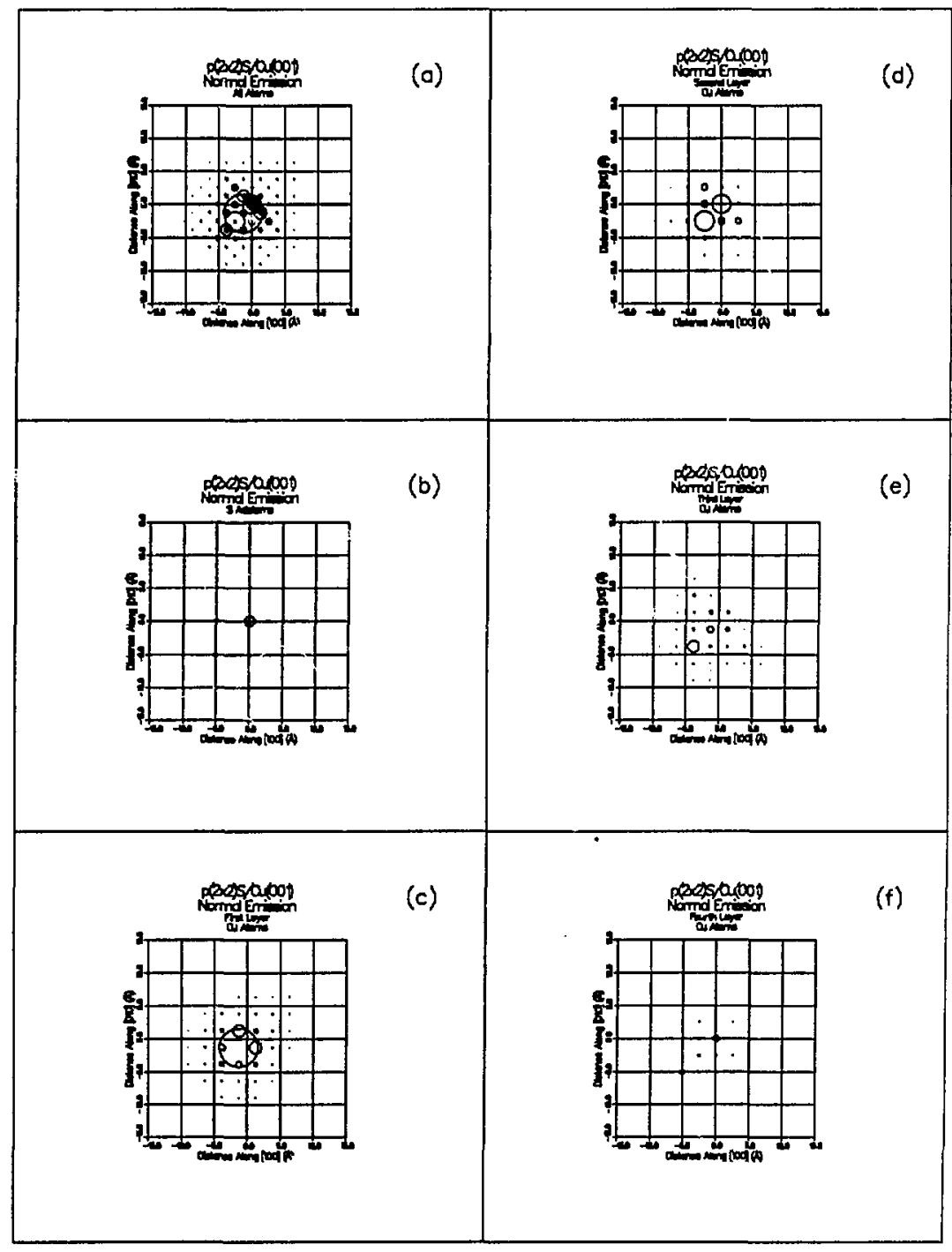


Figure 4.10:

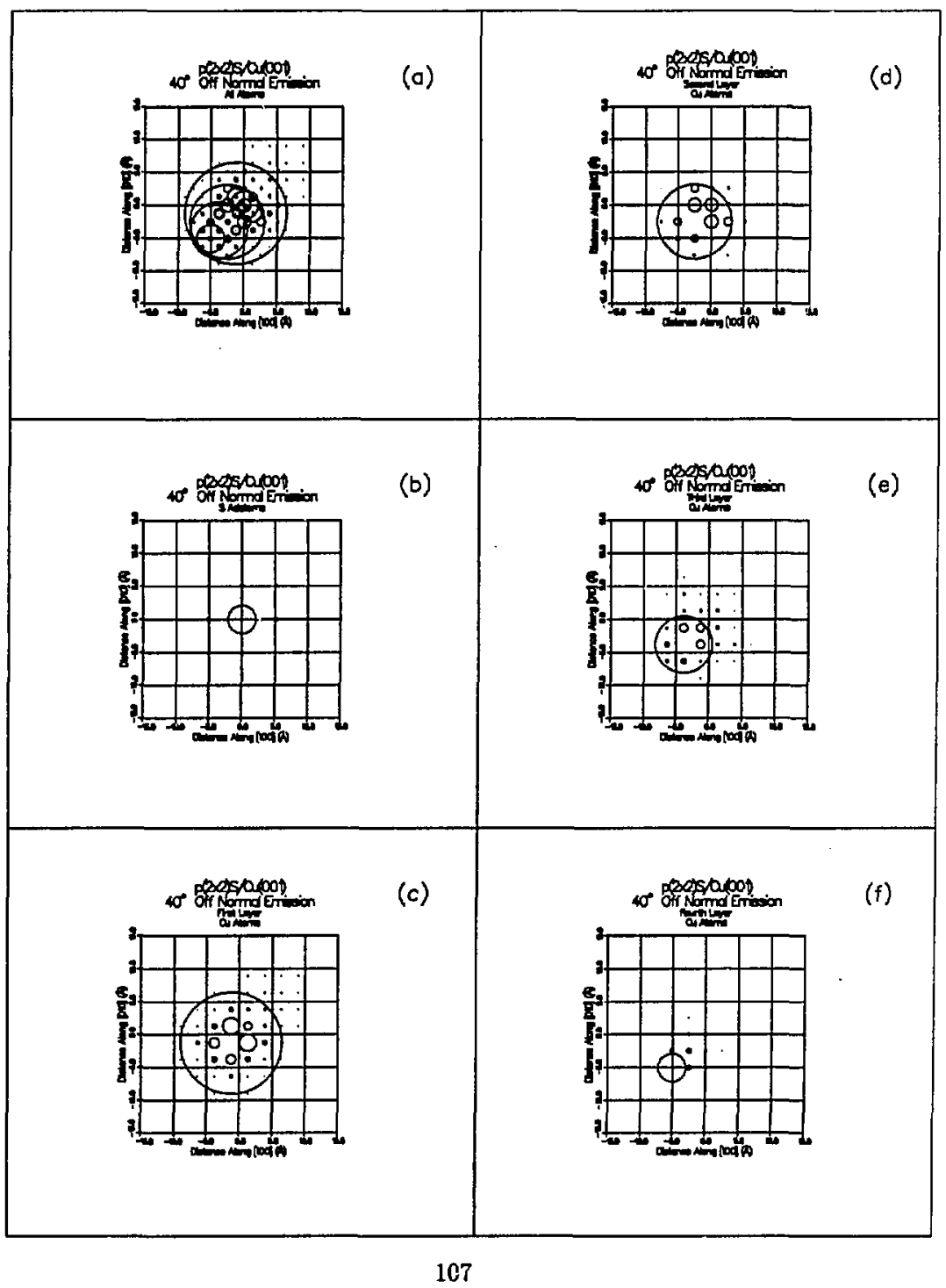




\section{Chapter 5}

\section{Effects of Surface Reconstruction in Valence Band Photoemission: Applications to $\mathrm{Au}(001)$}

\subsection{Abstract}

We have obtained valence band normal photoemission spectra from $\mathrm{Au}(001)-(1 \times 1)$ and $A u(001)-(5 \times 20)$ crystal surface reconstructions using photons ranging in energy from $9 \mathrm{eV}$ to $32 \mathrm{eV}$. The $\mathrm{Au}(001)-(1 \times 1)$ spectra consist almost entj-ely of direct transition features with few density of states peaks. Using the direct transition model and calculated bulk conduction bands as final states we derive an empirical valence band map for Au along the $\Gamma-X$ direction which is in good agreement with self-consistent band structure calculations. Empirical values of critical energies at $\Gamma$ and $X$ points are presented. Surface umklapp effects appear to be small. 


\subsection{Introduction}

Angle-resolved photoemission has been used to obtain valence band dispersion relations for a wide range of crystalline elements and compounds ${ }^{\text {(4) }}$. In most cases the experimentally obtained $\mathrm{E}(\mathrm{k})$ curves agree semi-quantitatively with theoretical predictions. The final state is sometimes taken to have the free electron form

$$
E\left(k_{f}\right)=\frac{\hbar^{2}\left(k_{i}+G\right)^{2}}{2 m^{*}}+V_{0}
$$

Here $k_{f}$ is the final state wavevector, $k_{i}$ is the initial state wavevector, and $G$ is a reciprocal lattice vector. The effective electron mass $m^{*}$ and the crystal potential $V_{0}$ are typically adjusted to maximize agreement between experiment and the calcuiated valence tands. In other cases the bulk conduction bands are used as final states, again with good agreement being reported.

The surface can affect the photoemission spectra in different ways. In $\operatorname{Ir}(001)^{55}$ it was found that surface order drastically affected the photoemission spectra. The changes observed in going from $\operatorname{Ir}(001)-(1 \times 1)$ to $\operatorname{Ir}(001)-(5 \times 20)$ were primarily a broadening of spectral peaks accompanied by a decrease in their intensities. These effects were attributed to surface umklapp processes, which would complicate bulk $k$ resolution by scattering excited electrons away from their primary emission directions. Changes in the photoemission spectra of $\operatorname{Ir}(001)$ after surface reconslauction were attributed to relaxation of bulk transition selection rules. This relaxation of bulk selection rules because of the surface does not appear very strong, as seen in a study of $\mathrm{Cu}(211)^{66}$. Although the symmetry group of the reconstructed surface is $C_{3}$, their spectra are seen to follow $C_{2 v}$ selection rules, as if the surface were not having any effect. A study of $\mathrm{Au}(001)$ with a He resonance lamp was interpreted to show that changes in the photoemission spectra upon surface reconstruction were due to relaxation of bulk selection rules ${ }^{67}$. 
As in Ir and Pt, the (001) face of Au has two surface orders: the metastable $(1 \times 1)$ in which the surface atoms retain their bulk positions (a square array) and the stable so-called $(5 \times 20)$ reconstruction. The latter is thought from LEED measurements to be an approximately square array on top of an essentially unchanged bulk structure. In Au this structure would more accurately be described as $c(26 \times 68)^{68,69}$, but we shall use the $(5 \times 20)$ notation.

We have carried out normal photoemission studies of $\mathrm{Au}(001)$ using monochromatic synchrotron radiation in the energy range $9 \mathrm{eV}-32 \mathrm{eV}$. By studying both the $(1 \times 1)$ and $(5 \times 20)$ reconstructions we could examine the influence of the surface on the photoemission spectra. In addition, we have determined the valence band dispersion relations for $\mathrm{Au}$ along most of the $\Gamma-\mathrm{X}$ line and compared the results with recent calculations ${ }^{70}$. Furthermore, because calculated conduction bands are available for Au we could tell what artifacts are introduced by using a plane wave final state. We note that a valence band map for the $\Gamma-X$ line in $\mathrm{Pt}$ showed severe distortions in the shapes of some of the bands when a plane wave final state was used $^{71}$.

\subsection{Experimental}

A high purity crystal of Au was cut to produce a (001) surface, polished to $6 \mu$ roughness, etched in aqua regia and then electropolished. The crystal was installed in an ultrahigh vacuum chamber and cleaned by a combination of repeated Ar ion sputtering and annealing cycles until Auger peak-to-peak intensities of $\mathrm{C}, \mathrm{S}$, and O were less than $1 \%$ each of the Au peak to peak intensity. Reconstructions were confirmed by LEED spectroscopy.

The photoemission measurements were performed on Beam Line 1-2 at the Stanford Syuchrotron Radiation Laboratory w:th the incident radiation linearly 
polarized (>97\%). The apparatus used has been described elsewhere ${ }^{21,22}$. The crystal was oriented in situ using laser autocollimation and the normal was determined to within $\pm 1^{\circ}$. The photons were incident at $55^{\circ}$ from the surface normal. Base pressure was less than $5 \times 10^{-10}$ torr. The energy resolution (monochromator plus analyzer) ranged from about $74 \mathrm{meV}$ FWHM at $\mathrm{h} \nu=9 \mathrm{eV}$ to $83 \mathrm{meV}$ at $\mathrm{h} \nu=32 \mathrm{eV}$. Analyzer angular resolution was $3^{\circ}$. Two sets of spectra were obtained with each surface reconstruction, one with the photon $E$ vector in the (101) plane and the other with the photon E vector in the (111) plane. Sample spectra are shown in Figure 5.1.

\subsection{Analysis}

\subsubsection{Selection Rules}

The spectra appear to obey relativistic dipole selection rules. With our experimental geometry the final state, a wave travelling in the ' $z$ ' direction, has $\Delta_{6}$ symmetry. With the photon electric field having both perpendicular and parallel components, initial states having either $\Delta_{6}$ or $\Delta_{7}$ symmetry should be observable ${ }^{72}$. In the $\Gamma-\mathrm{X}$ direction in $A u$ there are six such initial state bands and the spectra are consistent with six partially resolved peaks.

Non-relativistic selection rules would have led to a different result. With our experimental geometry, the final state would have symmetry $\Delta_{1}$ and the photon electric field would be $\Delta_{1}+\Delta_{5}$. In this case only initial states of symmetry $\Delta_{1}$ and $\Delta_{5}$ are allowed ${ }^{73}$ and the spectra would have at most three peaks, not the two to six that are observed. 


\subsubsection{Valence Band Structure}

\section{Theoretical Band Structure}

In Figure 5.2 we show theoretical ${ }^{70}$ valence and conduction bands of $\mathrm{Au}$ in the $\mathrm{\Gamma}-\mathrm{X}$ direction. All the valence bands are shown, but only the conduction bands with $\Delta_{6}$ symmetry are included (these are the only allowed final states, see Section 5.4.1 above). The bands have been scaled according to the formula

$$
E(k)=E^{\prime}(k)+1.06\left[E^{\prime}(k)-E_{F}\right]
$$

where $E(k)$ is the rescaled energy, and $E^{\prime}(k)$ is the calculated ${ }^{70}$ energy. In this fashion the scaled values become consistent with results of photoemission studies of $\mathrm{Au}(111)^{74}$. This is meant only as an empirical scaling; we note that de Haas-van Alphen ${ }^{75}$ measurements indicate that the upper valence band crosses the Fermi surface at $k=\frac{2 \pi}{3}(0,0,0.878)$, not at $\frac{2 \pi}{3}(0,0,845)$ as shown here. Our correction does not adjust for this.

\section{Experimental Band Structure, Plane Wave Final State}

As mentioned earlier, the free electron final state is frequently used to determine initial state dispersion relations. We found that several parabolas yielded band maps which agree with the calculated bands in different parts of the zone. Setting $\mathrm{m}^{*}$ to $0.98 \mathrm{~m}_{e}$ and $V_{o}$ to $-2.56 \mathrm{eV}$ in Equation 5.1 maximized agreement near $X$ in the Brillouin zone (Figure 5.3a), at the expense of agreement near the middle of the $\Gamma-\mathrm{X}$ line. In the middle of the $\Gamma-\mathrm{X}$ line the lowest two $\Delta_{6}$ conduction bands flatten and deviate from the quasi-free electron parabola (Figure 5.4). If the true final state(s) resembles the conduction bands, use of the quasi-free electron dispersion curve as the final state would cause serious discrepancies in the assumed reduced $k$ for the transition. In the plane wave band map (Figure 5.3a) several 
of the experimental bands exhibit a sudden sharp bend in the middle of the $\Gamma-\mathrm{X}$ line. No single parabola gave an experimental band map which agreed with the calculated bands over the entire zone, which suggests that several final states are necessary. We note that similar distortions were also obtained in $\operatorname{Pt}(001)^{71}$, where a plane wave final state was used in the valence band mapping.

\section{Experimental Band Structure, Conduction Band Final State}

Experimental bands were determined in the following fashion. If we take the scaled conduction bands to be the true final states, then by knowing both the photon energy and the binding energy of a given peak we can narrow down the reduced wavevector for the transition to at most three possible values, one for each of the conduction bands that may be the final state for the transition. For each transition at a given photon energy, then, we shift the final states down by that photon energy. This gives us at most three possible values $E(k)$ for an initial state. The one which comes closest in $k$ units to any of the valence bands is assumed to have been the initial state. In this way we evaluate the consistency of the calculated bands with the observed spectra. A valence band map derived in this way is shown in Figure 5.3b, along with the scaled theoretical bands. We see that with the exception of band 2 the agreement is good. A similar band map is derived from the $(5 \times 20)$ spectra.

We can, of course, use this process to look at the final states. In Figure 5.4 we show the band map that results when we show the transitions to the final states. We see that the portions of the conduction bands that are chosen by this evaluation-of-consistancy method lie quite close to the free electron parabola used in Figure 5.3a. This partially explains the success of the plane wave model for deriving dispersion relations. The free electron parabola mimics the lowest 
conduction band near the $X$ point, where errors in $k$ are most apparent for $E(k)$. Deviations near $\Gamma$ are not as important, as the valence bands are almost flat. Disagreement between valence bands thus derived and calculated bands in the middle of the $\Gamma-\mathrm{X}$ line has, however, been noted in the case of $\mathrm{Pt}(001)^{71}$. It is not surprising that only the parabolic stretches of the conduction bands are used, as the bulk final state must join reasonably well near the surface with the free electron state in the vacuum.

\subsubsection{Critical Point Energies}

From the valence band dispersion relations (Figure 5.3a or Figure 5.3b) we can estimate the energies of critical points at $\Gamma$ and $X$. Such a summary may be useful for comparison with inverse photoemission experiments. The critical points above the Fermi level can be determined by combining our results with published reflectivity measurements. For example, Olson ${ }^{76}$ identified $\Gamma_{8^{+}} \rightarrow \Gamma_{7^{-}}, \Gamma_{7^{+}} \rightarrow \Gamma_{7^{-}}$, and $\Gamma_{8+} \rightarrow \Gamma_{7^{-}}$transitions as having energies of $19.9 \mathrm{eV}, 21.2 \mathrm{eV}$, and $22.7 \mathrm{eV}$, respectively. This is consistent with a $\Gamma$ energy of $16.5 \mathrm{eV}$ above the Fermi level. Szczepanek ${ }^{77}$ identified $\mathrm{X}_{7+} \rightarrow \mathrm{X}_{6-}$ and $\mathrm{X}_{6+} \rightarrow \mathrm{X}_{6-}$ transitions having energies $3.1 \mathrm{eV}$ and $3.8 \mathrm{eV}$. Along with our results this puts the lowest $\mathrm{X}_{6}-$ level at $0.9 \mathrm{eV}$ above the Fermi level. The critical points which lie below the Fermi level can be taken directly from the diagram by extrapolation. These values along with theoretical results are summarized in Table 5.1.

\subsection{Umklapp Effects}

In Section 5.4 we noted the improvement in agreement between the theoretical bands and our data when the conduction bands were used as final states. In some ways this improvement is unsurprising, since we are in essence introducing more 
fitting parameters. The selection of final states, though, did show a physically reasonable result: only the parabola-like portions of the conduction bands were used.

We feel, though, that the simple algorithm used to do the band assignments may have been overly successful in assigning transitions in an effort to achieve consistence with the theoretical calculations.

Recall that in the Direct Transition Model used here, the reciprocal lattice vector was assumed to be unique. It was further assumed to be $\frac{2 \pi}{4}(0,0,2)$, i.e. the reciprocal lattice vector pointing into the analyzer. This was a slight simplification, any combination of crystal bulk or surface reciprocal lattice vectors may be used in the transition. We divide these reciprocal lattice vectors into two groups: bulk reciprocal lattice vectors and surface reciprocal lattice vectors.

Consider the bulk reciprocal lattice vectors of the set $\frac{2 \pi}{a}( \pm 1, \pm 1,1)$. In the extended zone scheme, the final state in this experiment has the form $k_{f}=\frac{2 \pi}{a}(0,0,2-x)$ (again, the free-electron form of Figure 5.4), where $0<x<1$. In this format, a vertical transition at $\Gamma$ corresponds to $x=0$ and a vertical transition at $\mathrm{X}$ corresponds to $\mathrm{x}=1$.

Now consider using the reciprocal lattice vector $G=\frac{2 \pi}{a}(-1,-1,1)$. If this vector is used for the transition, the initial wavevector would be $\frac{2 \pi}{\mathrm{a}}(1,1,1-\mathrm{x})$. A value of $x=0$ for the initial state corresponds to a state $k_{i}=\frac{2 \pi}{3}(1,1,1)$ (i.e. $\Gamma$ ) making the transition to $\frac{2 \pi}{3}(0,0,2)$ (i.e. $\Gamma$ ). Further, these reciprocal lattice vectors lead to a $\mathrm{X}$ - $\mathrm{X}$ transition. Thus, for our experimental geometry, we suspect that we are insensitive to whether the photoemission is so-called primary or secondary using bulk reciprocal lattice vectors.

Let us now consider surface reciprocal lattice vectors. These would be of the form $\frac{2 \pi}{a}( \pm m, \pm n, 0)$. Of particular interest is $\frac{2 \pi}{4}(-1,-1,0)$. A final state 
$\frac{2 \pi}{a}(0,0,2-x)$ could be reached using the bulk $\frac{2 \pi}{a}(0,0,2)$, the surface $\frac{2 \pi}{a}(-1,-1,0)$, and the initial $\frac{2 \pi}{2}(1,1,-x)$. Thus we have

\begin{tabular}{|c|c|c|c|}
\hline$x=0$ & $\begin{array}{c}\text { Initial State } \\
\frac{2 \pi}{(1,1,0)} \\
(\text { i.e. X) }\end{array}$ & $\rightarrow$ & $\begin{array}{c}\text { Final State } \\
\frac{2 \pi}{(0,0,2)} \\
(\text { i.e. } \Gamma)\end{array}$ \\
\hline$x=-\frac{1}{2}$ & $\begin{array}{l}\frac{2 \pi}{3}\left(1,1,-\frac{1}{2}\right) \\
(\text { i.e. } \Delta)\end{array}$ & $\rightarrow$ & $\begin{array}{c}\quad \frac{2 \pi}{(}\left(0,0,2-\frac{1}{2}\right) \\
(\text { i.e. } \Delta)\end{array}$ \\
\hline $\bar{x}$ & $\begin{array}{l}\frac{2 \pi}{(1,1,-1)} \\
(\text { i.e. } \Gamma)\end{array}$ & $\rightarrow$ & $\begin{array}{l}\frac{2 \pi}{(0,0,1)} \\
(\text { i.e. X) }\end{array}$ \\
\hline
\end{tabular}

Thus, surface umklapp processes would apparently 'reflect' the initial state bands about the center of the $\Gamma-X$ line. We see that the 'weak' features in Figure 5.3a seem to track the middle reflected bands in Figure 5.5, albeit with a shift in binding energy of $\sim 0.6 \mathrm{eV}$. These 'weak' features are quite small. In Figure 5.6 we show a close-up of one of these features.

\subsection{Conclusions}

We have obtained normal photoemission spectra from $A u(001)-(1 \times 1)$ and $A u(001)$ $(5 \times 20)$ using photons energies in the range $9 \mathrm{eV}-32 \mathrm{eV}$. Valence band maps derived from these spectra are consistent with the calculations of Eckhardt et al. The final states used in the direct transitions closely resemble the conduction bands with a large single plane wave component, with several conduction bands being used as final states in this energy range.

\subsection{Acknowledgements}

This work was supported by the Director, Office of Energy Research, Office of Basic energy Sciences, Chemical Sciences Division of the U.S. Department of Energy under Contract No. DE-AC03-76SF00098. It was performed at the Stanford Syn- 
chrotron Radiation Laborator: which is supported by the Department of Energy's Office of Basic Energy Sciences. 


\subsection{Table}

Table 5.1: Critical Point Energies for Au.

\begin{tabular}{c|cc|cc}
\hline & \multicolumn{2}{|c}{ Theory } & \multicolumn{2}{c}{ Experiment } \\
State & ref $^{78}$ & ref $^{70}$ & & \\
\hline$\Gamma_{8+}$ & -3.29 & -3.38 & $-3.55^{\mathrm{a}}$ & $-3.72^{\mathrm{c}}$ \\
$\Gamma_{7+}$ & -4.34 & -4.33 & $-4.45^{\mathrm{a}}$ & \\
$\Gamma_{8+}$ & -5.64 & -5.75 & $-5.90^{\mathrm{a}}$ & $-5.90^{\mathrm{c}}$ \\
$\Gamma_{7-}$ & 15.6 & 16.2 & $16.6^{\mathrm{b}}$ & $16.5^{\mathrm{e}}$ \\
$\mathrm{X}_{7+}$ & -1.53 & -1.72 & $-2.13^{\mathrm{c}}$ & \\
$\mathrm{X}_{6+}$ & -2.59 & -2.77 & $-2.94^{\mathrm{c}}$ & \\
$\mathrm{X}_{7+}$ & -2.77 & -3.00 & $-3.15^{\mathrm{c}}$ & \\
$\mathrm{X}_{7+}$ & -7.00 & -6.89 & $-6.49^{\mathrm{c}}$ & \\
$\mathrm{X}_{6+}$ & -7.39 & -7.27 & $-7.3^{\mathrm{c}}$ & \\
$\mathrm{X}_{6+}$ & 1.47 & 1.89 & $0.9^{\mathrm{d}}$ & \\
\hline
\end{tabular}

- reference $^{74}$.

${ }^{b}$ derived from ${ }^{74}$ and ${ }^{76}$.

c this work.

d derived from this work and reference ${ }^{77}$.

${ }^{e}$ derived from this work and reference ${ }^{76}$. 


\subsection{Figure Captions}

Figure 5.1: Typical normal valence band photoemission spectra of $A u(001)$.

a) Photoemission spectra from $\mathrm{Au}(001)-(1 \times 1)$. The photon $\mathrm{E}$ vector is in the [111] plane. The spectra range from $\mathrm{h} \nu=9 \mathrm{eV}$ at the top of the figure to $\mathrm{h} \nu=24 \mathrm{eV}$ at the bottom, in steps of $\Delta \mathrm{h} \nu=1.0 \mathrm{eV}$.

b) Photoemission spectra from $A u(001)-(1 \times 1)$. The photon $E$ vector is in the [011] plane. The spectra range from $h \nu=9 \mathrm{eV}$ at the top of the fipure to $\mathrm{h} \nu=32 \mathrm{eV}$ at the bottom, in steps of $\Delta \mathrm{h} \nu=1.0 \mathrm{eV}$. There is no spectrum for $\mathrm{h} \nu=27 \mathrm{eV}$.

c) Photoemission spectra from $\mathrm{Au}(001)-(5 \times 20)$. The photon $\mathrm{E}$ vector is in the [111] plane. The spectra range from $h \nu=9 \mathrm{eV}$ at the top of the figure to $\mathrm{h} \nu=32 \mathrm{eV}$ at the bottom, in steps of $\Delta \mathrm{h} \nu=1.0 \mathrm{eV}$.

d) Photoemission spectra from $\mathrm{Au}(001)-(5 \times 20)$. The photon $\mathrm{E}$ vector is in the [011] plane. The spectra range from $\mathrm{h} \nu=9 \mathrm{eV}$ at the top of the figure to $\mathrm{h} \nu=32 \mathrm{eV}$ at the bottom, in steps of $\Delta \mathrm{h} \nu=1.0 \mathrm{eV}$.

Figure 5.2: Theoretical Au valence bands, from reference ${ }^{70}$. All of the valence bands are shown, but only the $\Delta_{6}$ conduction bands are shown. Symmetry labels for the bands and critical points are also included.

Figure 5.3: Empirical valence band maps of $\mathrm{Au}(001)$, determined from the $\mathrm{Au}(001)-(1 \times 1)$ data. Open symbols denote weak features. The arrows mark the point at which the upper valence band crosses the Fermi level, as determined by de Haas-van Alphen measurements.

a) Band map obtained using a single plane wave final state. Note the apparent distortion of the middle $\Delta_{6}$ band in the middle of the $\Gamma-\mathrm{X}$ line.

b) Band map obtained using the $\Delta_{6}$ conduction bands as final states. We 
see that there is improved agreement between the data and the slope of the middle $\Delta_{6}$ line.

Figure 5.4: Free electron parabola (dotted line) with scaled conduction bands. The portions of the conduction bands that were used as final states are marked by crosses.

Figure 5.5: The weak features in Figure 5.3a resemble two of the valence bands, as they would appear if reflected about the cent of the $\Gamma-\mathrm{X}$ line. The reflected (and shifted upwards by $0.6 \mathrm{eV}$ ) bands are shown as dashed lines.

Figure 5.6: Normal emission spectrum from $A u(001)-(1 \times 1), h \nu=17 \mathrm{eV}$, photon $\mathbf{E}$ vector in [011] plane. The vertical lines indicate the peak positions; the arrow shows the relative size of the 'weak features' in Figure 5.3. 
Figure 5.1:

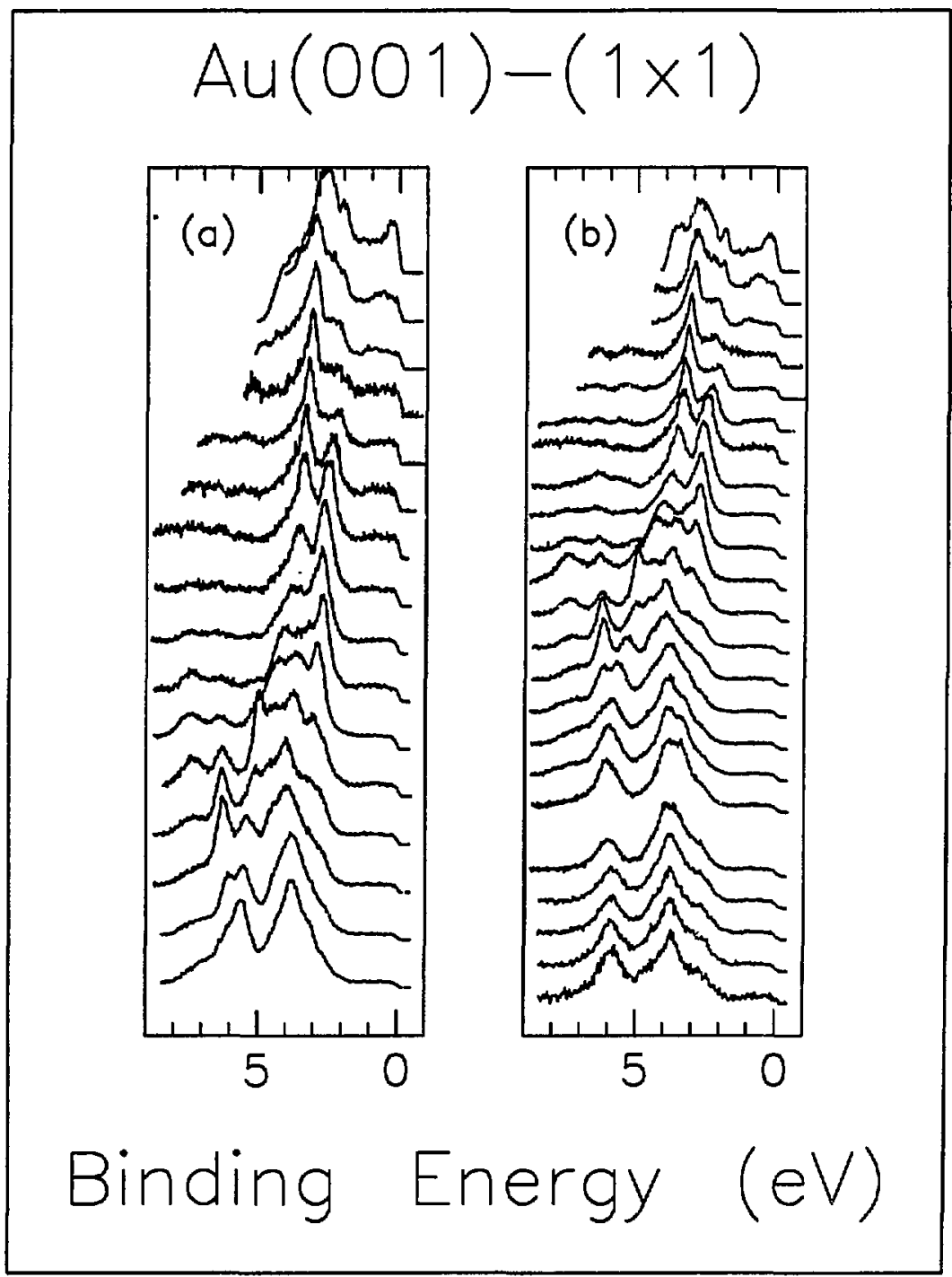


Figure 5.1:

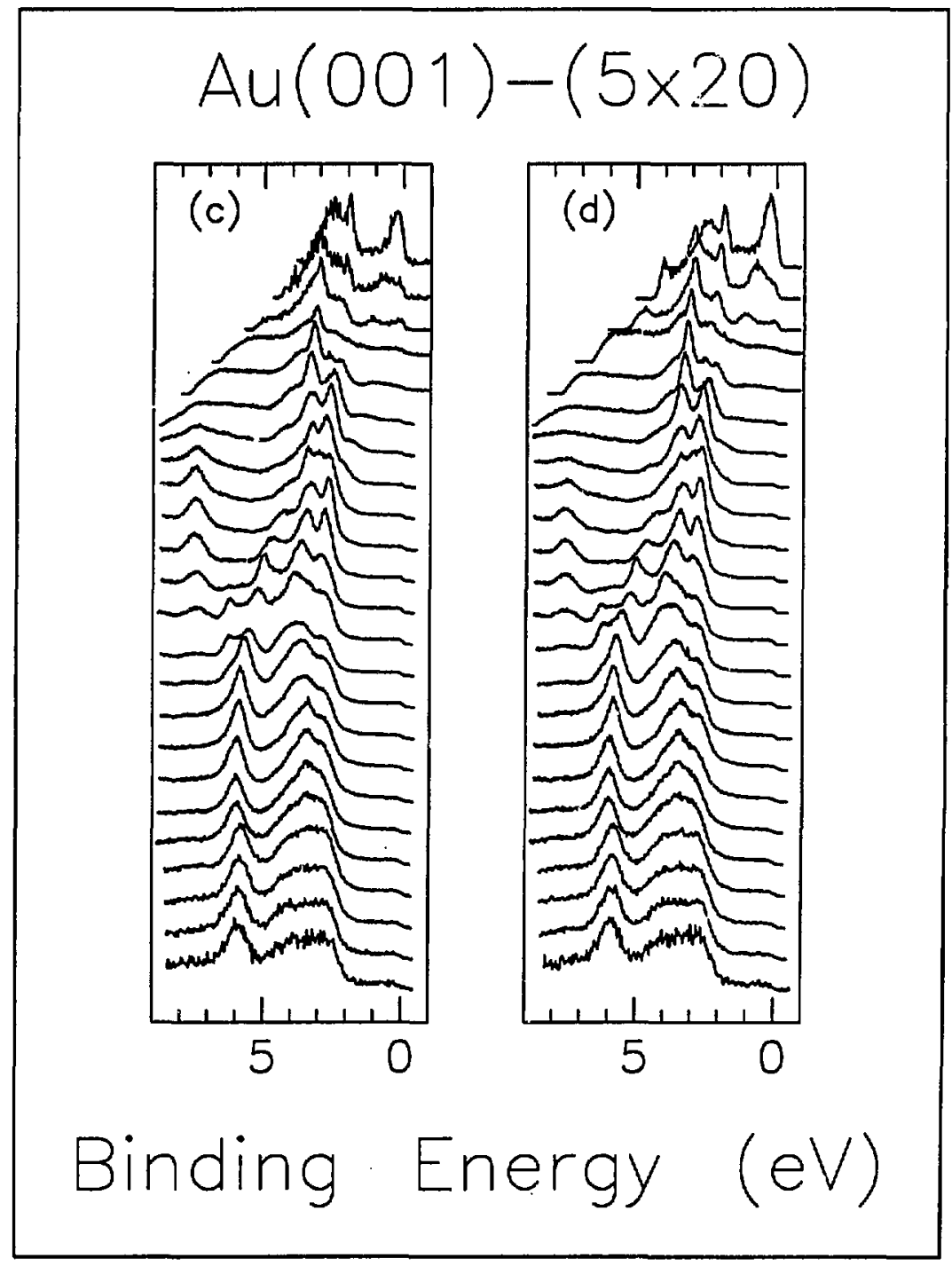


Figure 5.2:

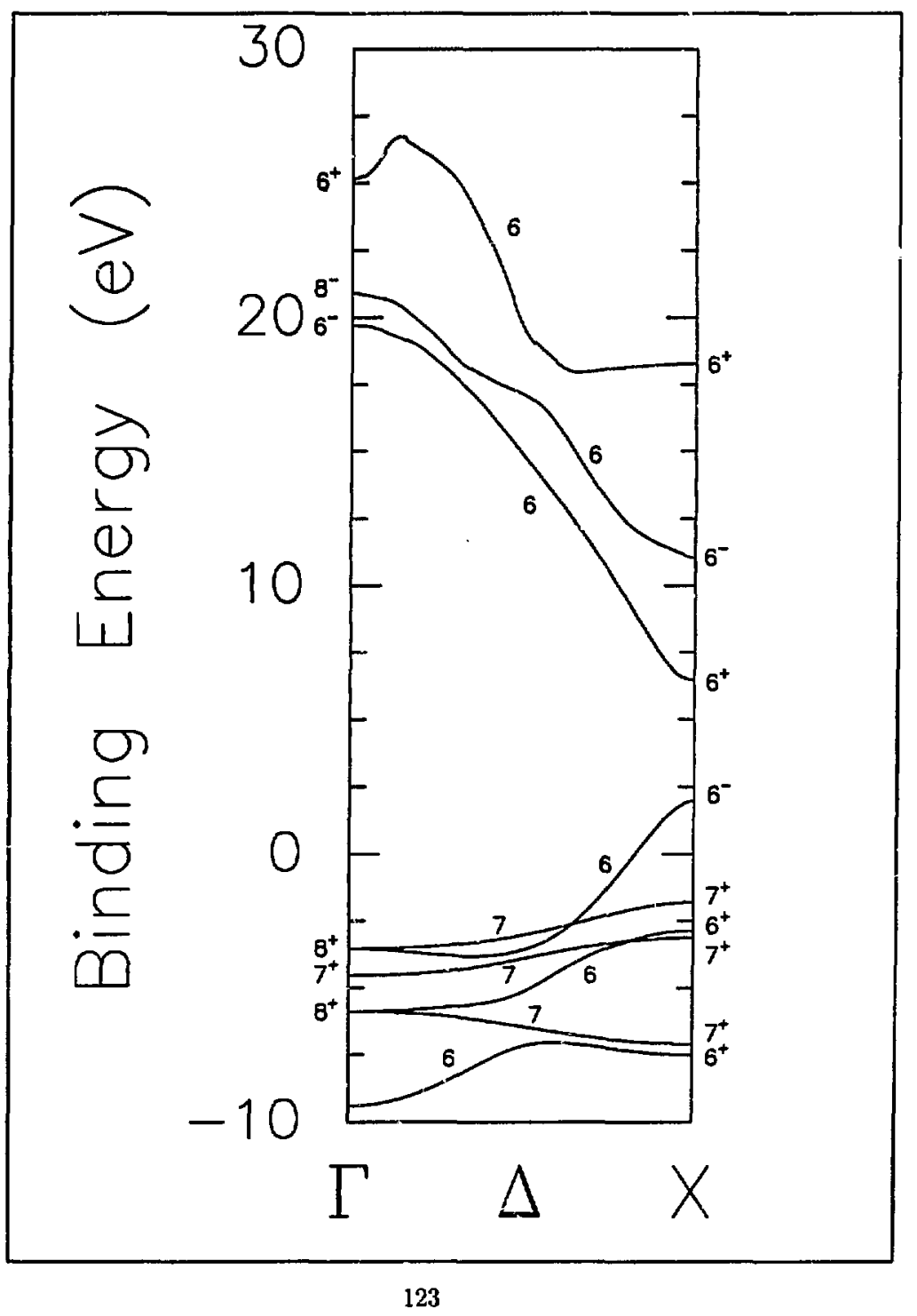


Figure 5.3:

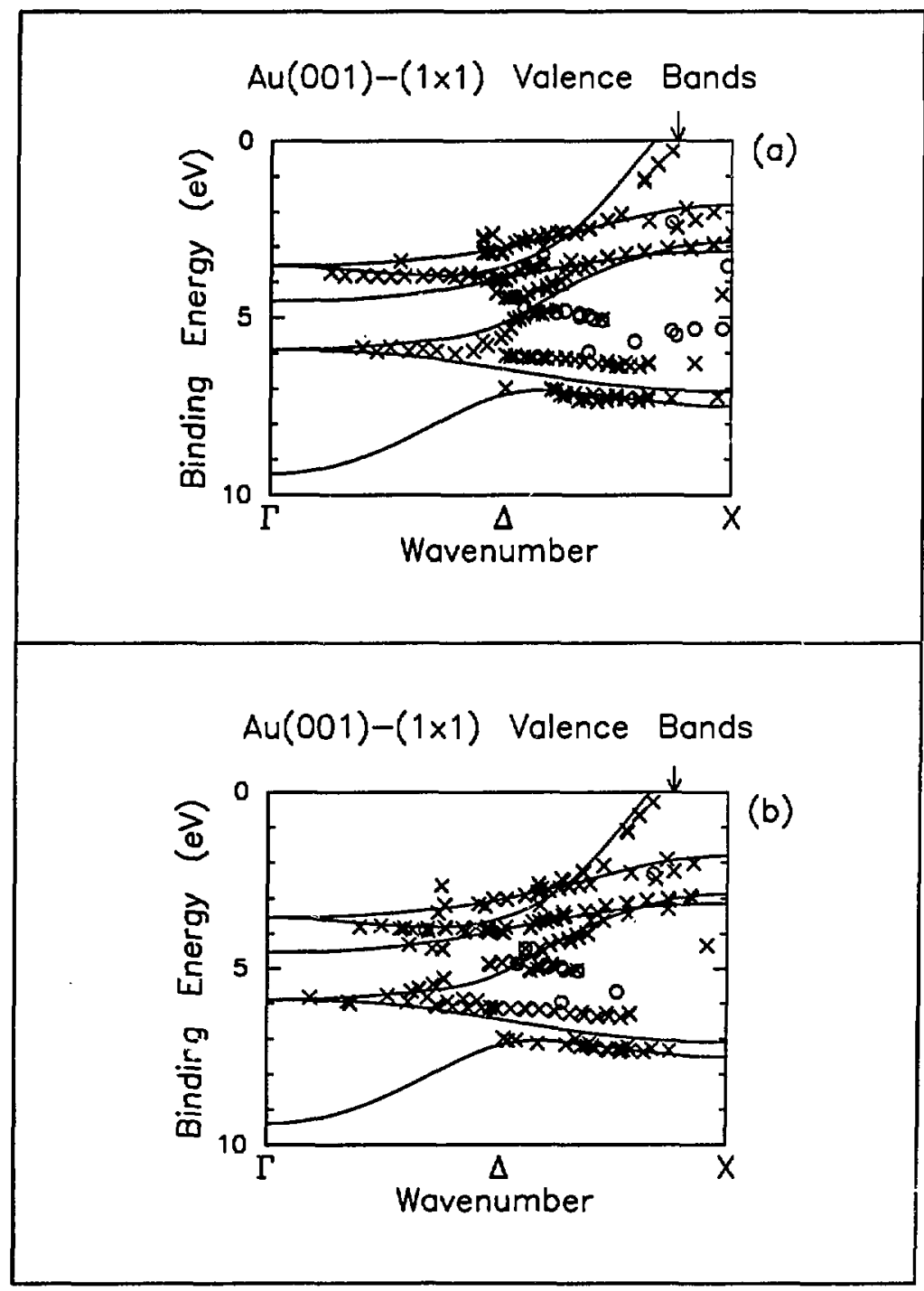




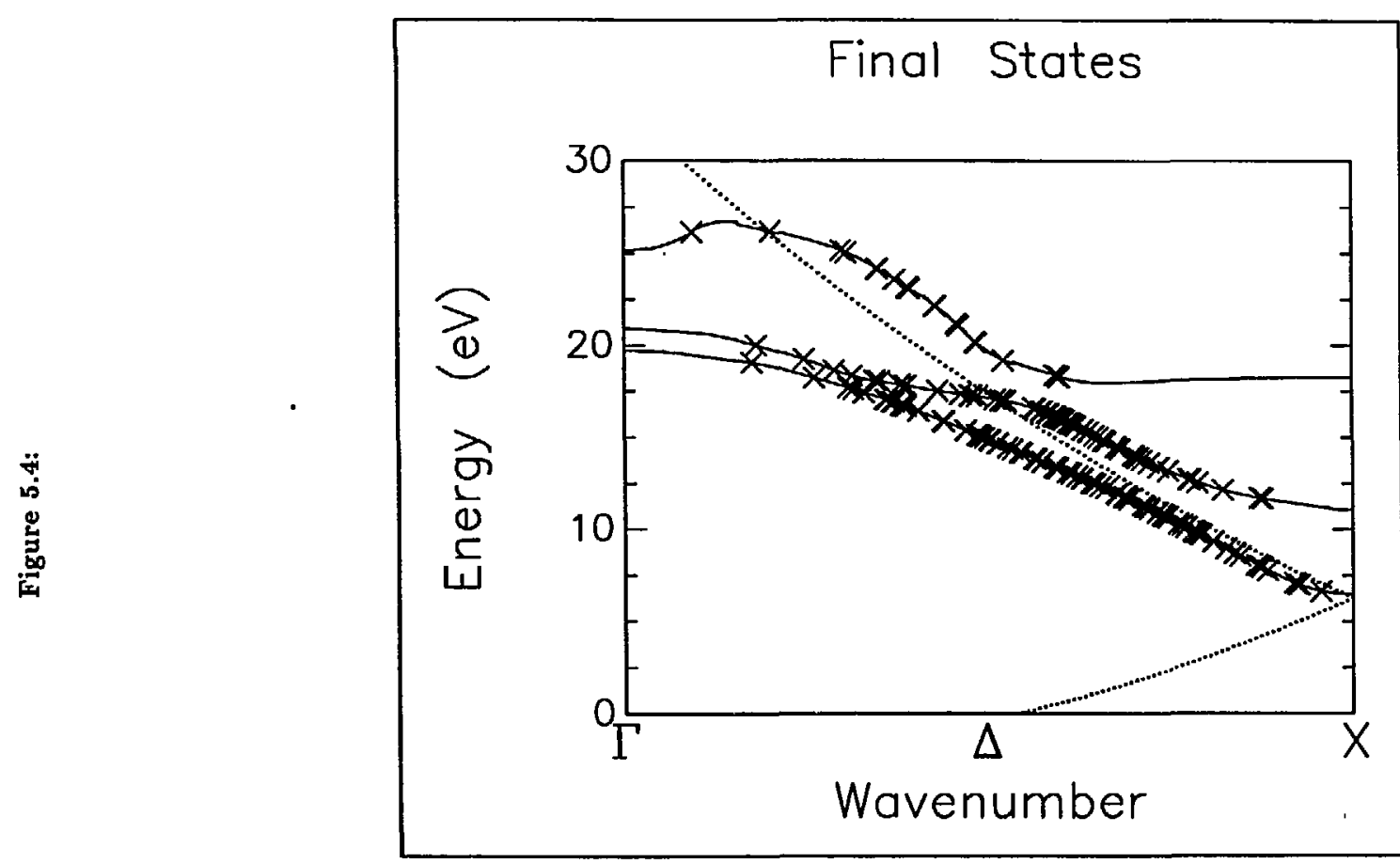


Figure 5.5:

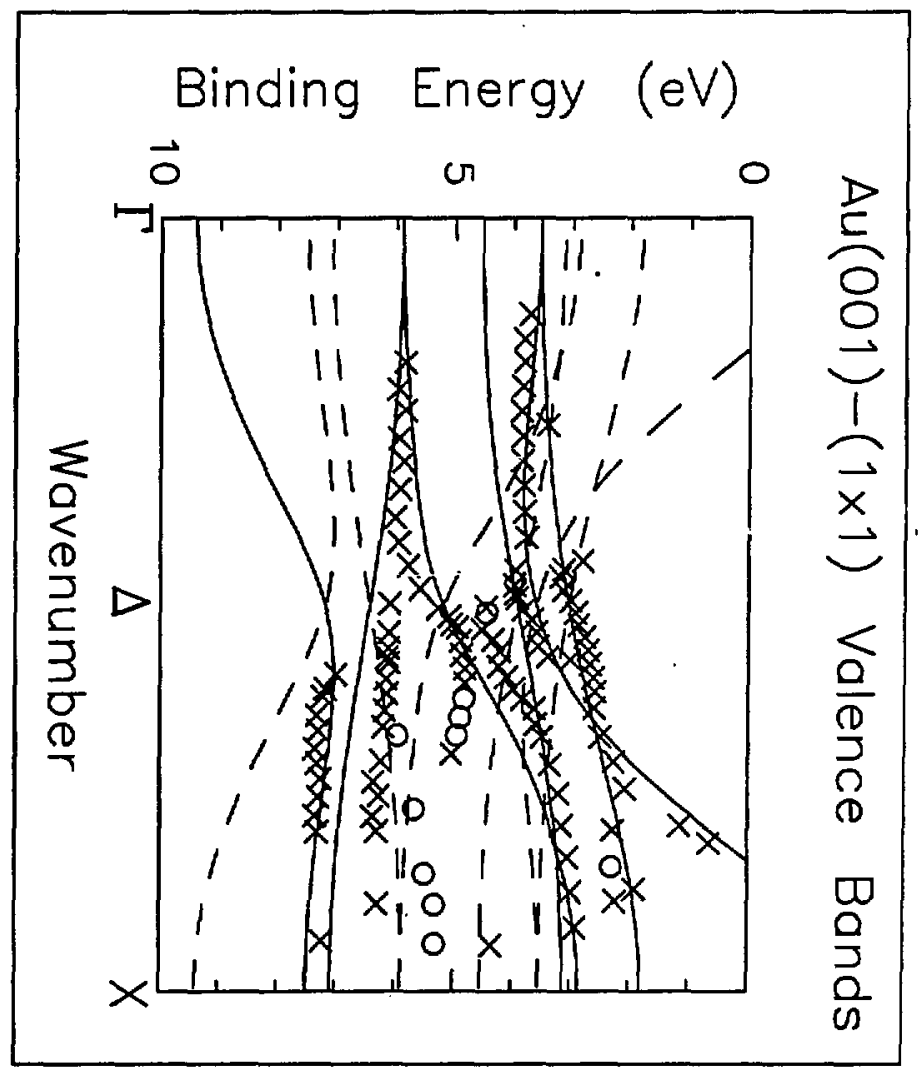


Figure 5.6:

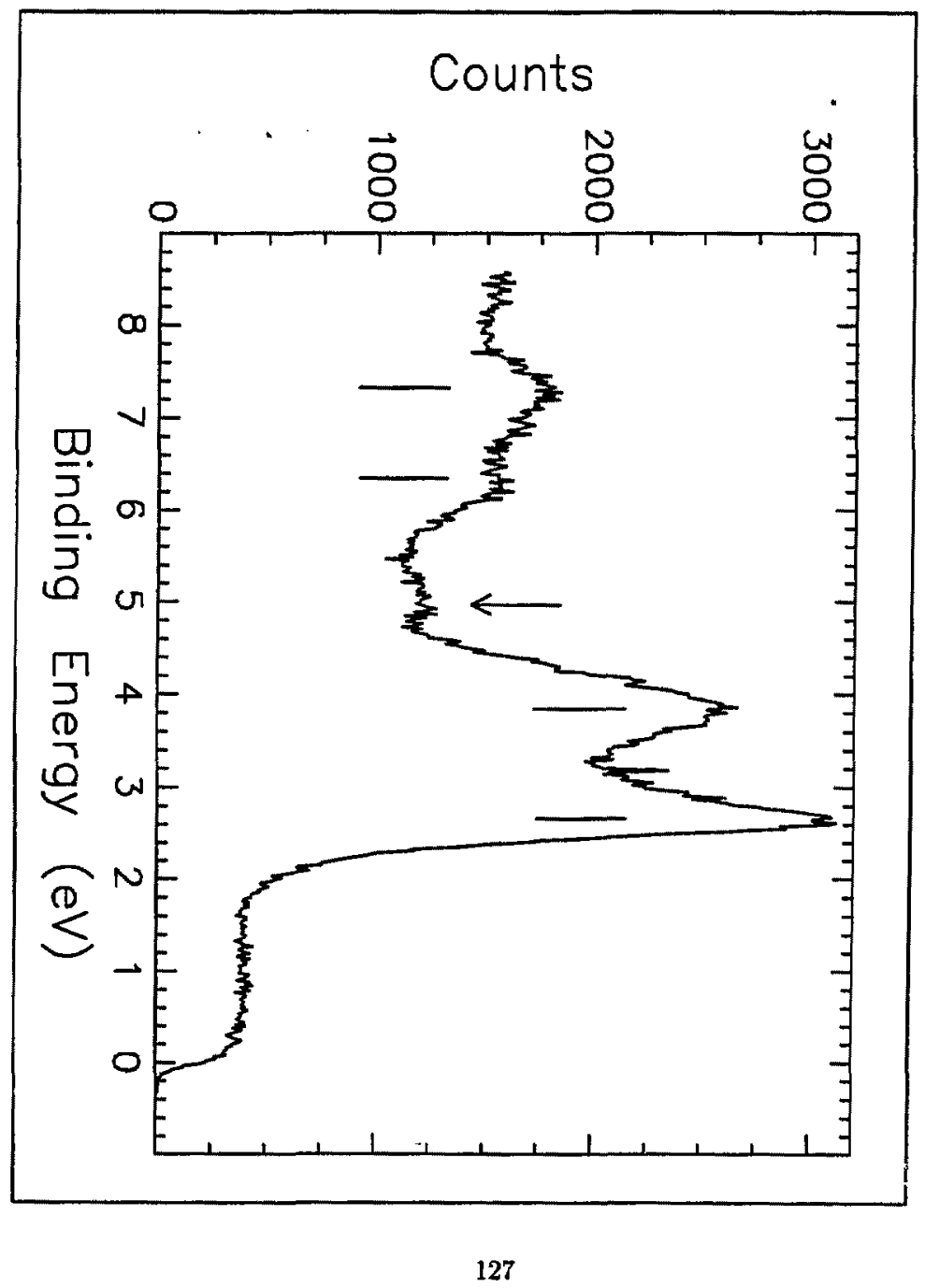




\section{Chapter 6}

\section{Conclusions}

Because of the debate over the $\mathrm{p}(2 \times 2) \mathrm{S} / \mathrm{Cu}(001)$ structure and the apparent error in the intial ARPEFS $p(2 \times 2) S / C u(001)$ result, we have upgraded both the experimental procedures for ARPEFS and the algorithm used to extract a structure. Repeating, the experimental upgrade was the modification of our equipment so that round-the-clock ARPEFS measurements could be made on a chilled system. While this complicates the data aquisition during a run (there is yet more to watch, and the loss of temperature stability can lead to the rejection of a $\chi(\mathrm{k})$ curve data set), the resulting increase in the signal-to-nois: ratio (Figure 2.13) is worth the additional effort. The other upgrade in the AFPEFS technique has been the implementation of the simplex algorithm to extract structural information from the data. This reduces human effort in the fitting of any one curves and facilitates multiple restarts of the fitting process; a useful precaution against stopping in a local minimum. We saw that this algorithmic change resulted in a better fit for previous $\mathrm{p}(2 \times 2) \mathrm{S} / \mathrm{Cu}(001)$ data (Figure 2.14). We point out that the simplex algorithm is not foolproof, Figure 3.3a shows an apparent failure in convergence.

From the results of $\mathrm{p}(2 \times 2) \mathrm{S} / \mathrm{Cu}(001)$, we have found that the data takingprocess is quite reproducible, as judged by the agreement between the normal emission curves taken on different beam lines under vastly different operating con- 
ditions (Figure 4.4). We have further found that the structures obtained are reproducible, as judged by the agreement in the fitted results to the two normal emission curves of $\mathrm{p}(2 \times 2) \mathrm{S} / \mathrm{Cu}(001)$. This would seem to rule out data-taking or data-reduction artifacts as sources of severe error in the ARPEFS technique.

ARPEFS has been used to assign structures to over a dozen adsorbate systems (it has been applied to a few more, with no compelling structural determination resulting). In Table 6.1 we show bond lengths for systems to which ARPEFS, LEED, and SEXAFS have all assigned values. There is a clear pattern: ARPEFS bond lengths are in between the LEED and SEXAFS bond lengths in all cases, and are usually one-third of the way between the LEED and SEXAFS values. Furthermore, with one exception, the LEED value is slightly shorter than the ARPEFS value, which in turn is shorter than the SEXAFS result.

The samples studied for this thesis, while useful to confirm that systematic differences exist, are unfortunately poor choices for a thorough study of the differences between LEED and ARPEFS. This seems due to the many degrees of freedom in the near-surface structural parameters. The $\mathrm{p}(2 \times 2) \mathrm{S} / \mathrm{Cu}(001)$ structure has six structural degrees of freedom, with two of these describing relation in the first layer. The experience with $\mathrm{p}(2 \times 2) \mathrm{S} / \mathrm{Ni}(111)$ and $\mathrm{p}(2 \times 2) \mathrm{S} / \mathrm{Cu}(001)$ shows that this, combined with the emission angle uncertainty, overwhelms the structure determination process. Since ARPEFS is unable to assign a precise structure to these samples, it naturally follows that they are poor standards for an inter-technique comparison. Samples which by symmetry have no surface reconstruction $\left(\mathrm{c}(2 \times 2) \mathrm{Cl} / \mathrm{Cu}(001),(\sqrt{3} \times \sqrt{3}) \mathrm{R} 30^{\circ} \mathrm{Cl} / \mathrm{Ni}(111)\right)$ which were studied with the techniques developed for this thesis (i.e. as low temperature as possible to maximize the signal-to-noise ratio, combined with simplex fitting of the resulting curves), appear quite amenable to an ARPEFS analysis. 
We are at a loss about the disagreement within the second layer reconstruction for $\mathrm{p}(2 \times 2) \mathrm{S} / \mathrm{Cu}(001)$, but one point bears mentioning. This is that the ARPEFS result was obtained at low temperatures. This effectively freezes out vibrations, enhancing the signal-to-noise ratio in the $\chi(\mathrm{k})$ curves. Trial calculations indicated that room temperature ARPEFS measurements would not compellingly resolve the differences in the second layer reconstruction. This may have affected the LEED result, although not being LEED experts we do not state this definitively. We also note that LEED experiments treat the relative intensities of data and theory curves as an adjustable parameter, whereas ARPEFS does not. This may be an additional complication for LEED. It would have added an extra fitting parameter to the ARPEFS $p(2 \times 2) S / C u(001)$ analysis, complicating an already lengthy data reduction process. This non-adjustability of the relative amplitudes is what led to our rejection of all assumed structures for $(\sqrt{3} \times \sqrt{3}) \mathrm{R} 30^{\circ} \mathrm{S} / \mathrm{Ni}(111)$, the FCC structure in particular.

To resolve/improve the agreement between LEED and ARPEFS, we suggest that both methods be applied to a chilled sample. We would suggest that this sample be some sort of $c(2 \times 2)$ overlayer on a (001) face; because of symmetry, these structures do not have surface layer reconstruction. 


\subsection{Table}

Table 6.1: Comparison of bond lengths for systems studied by ARPEFS, compared to the same parameters as determined by LEED and SEXAFS.

\begin{tabular}{c|ccc}
\hline System & LEED & ARPEFS & SEXAFS \\
\hline $\mathrm{p}(2 \times 2) \mathrm{S} / \mathrm{Cu}(001)$ & $2.23^{\mathrm{a}}$ & $2.26^{\mathrm{b}}$ & $2.31^{\mathrm{c}}$ \\
$\mathrm{c}(2 \times 2) \mathrm{S} / \mathrm{Ni}(001)$ & $2.19^{\mathrm{d}}$ & $2.19^{\mathrm{e}}$ & $2.23^{\mathrm{f}}$ \\
$\mathrm{c}(2 \times 2) \mathrm{S} / \mathrm{Ni}(011)$ & $2.32^{\mathrm{s}}$ & $2.31^{\mathrm{h}}$ & $2.23^{\mathrm{j}}$ \\
$\mathrm{p}(2 \times 2) \mathrm{S} / \mathrm{Ni}(111)$ & $2.10^{\mathrm{j}}$ & $2.13^{\mathrm{b}}$ & $2.20^{\mathrm{k}}, 2.23^{\mathrm{i}}$ \\
$\mathrm{c}(2 \times 2) \mathrm{Cl} / \mathrm{Cu}(001)$ & $2.41^{\mathrm{l}}$ & $2.42^{\mathrm{m}}$ & $2.37^{\mathrm{n}}$ \\
\hline
\end{tabular}

reference ${ }^{14}$

b this work

c reference ${ }^{11}$

d $_{\text {reference }}{ }^{79}$

- reference $^{80}$

1 reference ${ }^{81}$

reference ${ }^{82}$

${ }^{h}$ reference $^{3}$

i reference 46

${ }^{j}$ reference ${ }^{41}$

${ }^{k}$ reference ${ }^{42}$

1 reference ${ }^{83}$

$m$ reference ${ }^{57}$

${ }^{n}$ reference $^{84}$ 


\section{References}

'J.J. Barton, Ph.D. thesis, University of California, Berkeley 1985.

${ }^{2}$ C.C. Bahr, J.J. Barton, Z. Hussain, S.W. Robey, J.G. Tobin, and D.A. Shirley, Phys. Rev. B 35, 3773, (1987).

${ }^{3}$ S.W. Robey, J.J. Barton, C.C. Bahr, G. Liu, and D.A. Shirley, Phys, Rev. B 35, 1108, (1987).

${ }^{4}$ L.J. Terminello, X.S. Zhang, Z.Q. Huang, S. Kim, A.E. Schach von Wittenau, K.T. Leung, and D.A. Shirley, Phys. Rev. B 38, 3879, (1988).

${ }^{5}$ X.S. Zhang, L.J. Terminello, A.E. Schach von Wittenau, S.H. Kim, Z.Q. Huang, Z.Z Yang, D.A. Shirley, F.M. Tao, and Y.K. Pan, in prep.

${ }^{6}$ X.S. Zhang, L.J. Terminello, S. Kim, A.E. Schach von Wittenau, and D.A. Shirley, J. Chem. Phys. 89, 6538, (1988).

${ }^{7}$ S.W. Robey, C.C. Bahr, Z. Hussain, J.J. Barton, K.T. Leung, J.R. Lou, A.E. Schach von Wittenau, and D.A. Shirley, Phys. Rev. B 35, 5657, (1987).

${ }^{8}$ C.C. Bahr, S.W. Robey, Z. Hussain, L.T. Terminello, K.T. Leung, J.R. Lou, A.E. Schach von Wittenau, and D.A. Shirley, LBL Report \#21221, (1987).

${ }^{9}$ L.J. Terminello, K.T. Leung, Z. Hussain, T. Hayashi, and D.A. Shirley, Phys. Rev. B 41, 12787, (1990). 
${ }^{10}$ K.T. Leung, L.J. Terminello, , Z. Hussain, X.S. Zhang, T. Hayashi, and D.A. Shirley, Phys. Rev. B 38, 8241, (1988).

${ }^{11}$ J.R. Patel, D.W. Berreman, F. Sette, P.H. Citrin, J.E. Rowe, P.L. Cowan, T. Jach, añd B. Karlin, Phys. Rev. B 40, 1330, (1989).

${ }^{12}$ H.C. Zeng, R.N.S Sodhi, and K.A.R. Mitchell, Surf. Sci. 177, 329, (1986).

${ }^{13}$ H.C. Zeng, R.A. MacFarlane, and K.A.R. Mitchell, Phys. Rev. B 39, 8000 , (1989).

${ }^{14}$ H.C. Zeng, R.A. MacFarlane, and K.A.R. Mitchell, Can. J. Phys. 68, 353, (1990).

${ }^{15}$ E. Vlieg, I.K. Robinson, and R.McGrath, Phys. Rev. B 41, 7896, (1990).

${ }^{16}$ Z.Q. Wu, M.L. Xu, Y. Chen, S.Y. Tong, M.H. Mohamed, and L.L Kesmodel, Phys. Rev. B 36, 9329, (1987).

${ }^{17}$ Q.T. Jiang, P. Fenter, and T. Gustafsson, Phys. Rev. B 42, 9291, (1990).

${ }^{18}$ E.A. Stern, D.E. Sayers, and F.W. Lytle, Phys. Rev. B 11, 4836, (1975).

${ }^{19}$ R.B. Gregor and F.W. Lytle, Phys. Rev. B 20, 4902, (1979).

${ }^{20}$ E. Sevillano, H. Meuth, and J.J. Rehr, Phys. Rev. B 20, 4908, (1979).

${ }^{21}$ S.D. Kevan, Ph.D. thesis, University of California, Berkeley (19S0).

${ }^{22}$ S.D. Kevan and D.A. Shirley, Phys. Rev. B 22, 542, (1980).

${ }^{23}$ Z. Hussain, E. Umbach, D.A. Shirley, J. Stoehr, and J. Feldhaus, Nucl. Instrum. Methods 195, 115, (1982).

${ }^{24}$ P.L. Cowan, S. Brennan, R.D. Deslattes, A. Henins, T. Jach, and E.G. Kessler, Nucl. Instrum. Methods Phys. Res. Sect. A 246, 154, (1986). 
${ }^{25}$ J.J. Barton, S.W. Robey, and D.A. Shirley, Phys. Rev. B 34, 778, (1986).

${ }^{26}$ P.A. Flinn, G.M. McManus, and J.A. Rayne, Phys. Rev. B 123, 809, (1961).

${ }^{27}$ D.L. Adams and U. Landman, Phys. Rev. Bं 15, 3775, (1977).

${ }^{28}$ R. Trehan and C.S. Fadley, Phys. Rev. B 34, 6784, (1986).

${ }^{29}$ L.F. Mattheiss, Phys. Rev. 113, A134, (1964).

${ }^{30}$ T.L. Loucks, Augmented Plane Wave Method, (W.A. Benjamin), 1967.

31.B. Mann, Los Alamos National Laboratory Report \#3691, (1968).

${ }^{32}$ G. Frohnmeyer and R. Glocker, Acta Cryst. 6, 19, (1953).

${ }^{33}$ T.A. Hahn, J. Applied Physics 41, 5096, (1970).

${ }^{34}$ J.F. Janak, A.R. Williams, and V. L. Moruzzi, Phys. Rev. B 6, 4367, (1972).

${ }^{35}$ B.K. Teo, EXAFS, Basic Principles and Data Analysis, (Springer), 1986.

${ }^{36}$ J.M. Parkinson and D. Hutchinson, in Numerical Methods for Non-linear Optimization, (Academic Press), 1971.

${ }^{37}$ J.A. Nelder and R. Mead, Computer Journal 7, 308, (1965).

${ }^{38}$ W.H. Press, B.P. Flannery, S.A. Teukolsky, and W.T. Vetterling, Numerical Recipes, (Cambridge University Press) 1989.

${ }^{39}$ F.W. Lytle, D.E. Sayers, and E.A. Stern, Physica B 158, 701, (1988).

${ }^{40}$ P.R. Bevington, Data Reduction and Error Analysis for the Physical Sciences, (McGraw-Hill) 1969.

${ }^{41}$ Y.K. Wu and K.A.R. Mitchell, Can. J. Chem. 67, (1975, (1989). 
${ }^{42}$ T. Yokoyama, M. Funabashi, Y. Kitajima, T. Ohta, and H. Kuroda, Physica B 158, 643, (1989).

${ }^{13}$ T.W. Capehart, C.W. Seabury, G.W. Graham, and T.N. Rhodin, Surf. Sci. Letters 120, L441, (1982).

${ }^{14}$ D.R. Warburton, P.L. Wincott, G. Thornton, F.M. Quinn, and D. Norman, Surf. Sci. 211/212, 71, (1989).

${ }^{45}$ T. Fauster, H. Dürr, and D. Hartwig, Surf. Sci. 178, 657, (1986).

${ }^{46}$ D.R. Warburton, P.L. Wincott, G. Thornton, D. Norman, C.H. Richardson, F.M. Quinn, and R. McGrath, Vacuum 38, 241, (1988).

${ }^{47}$ J.E. Demuth, D.W. Jepsen, and P.M. Marcus, Phys. Rev. Lett. 32, 1182, (1974).

${ }^{48}$ J.B. Benziger and R.E. Preston, Surf. Sci. 141, 567, (1974).

${ }^{49}$ W. Erley and H. Wagner, J. Catalysis 53, 287, (1978).

${ }^{50}$ T. Edmonds, J.J. McCarroll, and R.C. Pitkethly, J. Vacuum Sci. Technology 8, $68,(1971)$.

${ }^{51}$ G.E. Becker and H.D. Hagstrom, Surf. Sci. 30, 505, (1972).

${ }^{52}$ P. Delescluse and A. Masson, Surf. Sci. 100, 423, (1980).

${ }^{53}$ Y.Kitajima, T. Yokoyama, T. Ohta, M. Funabashi, N. Kosugi, and H. Kuroda, Surf. Sci. Letters 214, L261, (1989).

${ }^{54}$ T.W. Capehart and T.N. Rhodin, Solid State Comm . 44, 7, (1982).

${ }^{35}$ A.E. Schach von Wittenau, L.Q. Wang, Z. Hussain, Z.Q. Huang, Z.G. Ji, and D.A. Shirley, in prep. 
${ }^{56}$ A.E. Schach von Wittenau, L.Q. Wang, Z. Hussain, Z.Q. Huang, and D.A. Shirley, in prep.

${ }^{57}$ L.Q. Wang, A.E. Schach von Wittenau, Z.G. Ji, L.S. Wang, T. Shulman, and D.A. Shirley, Phys. Rev. B 43, xxxx, (1991).

${ }^{58}$ J.J. Barton, Phy . Rev. B 32, (1906, (1985).

${ }^{69}$ J.B. Pendry, Low Energy Electron Diffraction, (Academic Press) 1974.

${ }^{60}$ J.C. Ashley and C.J. Tung, Surf. Int. Anal. 4, 52, (1982).

${ }^{61}$ M.P. Seah, Surf. Sci. 32, 703, (1972.

${ }^{62}$ M.A. Burke and J.J Schreuers, Surf. Int. Anal. 4, 42, (1982).

${ }^{63}$ D.M. Himmelblau, in Numerical Methods for Non-linear Optimization, (Academic Press) 1971.

${ }^{64}$ F.J. Himpsel, Ac். Phys. 32, 1, (1983).

${ }^{65}$ J.F. van der Veen, F.J. Himpsel, and D.E. Eastman, Phys. Rev. B 22, 4226, (i980).

${ }^{66}$ R.F. Davis, R.S. Williams, S.D. Kevan, P.S. Wehner, and D.A. Shirley, Phys. Rev. B 31, 1997, (1985).

${ }^{67}$ P. Heimann, J. Hermanson, H. Miosga, and H. Neddermeyer, Phys. Rev. Lett. 43, 1757, (1979).

${ }^{68}$ M.A. van Hove, R.J. Koestner, P.C. Stair, J.P. Biberian, L.L. Kesmodel, I. Bartos, and G.A. Somorjaj, Surf. Sci. 103, 189, (1981). 
${ }^{69}$ M.A. van Hove, R.J. Koestner, P.C. Stair, J.P. Biberian, L.L. Kesmodel, I. Bartcs, and G.A. Somorjai, Surf. Sci. 103, 21E, (1981).

${ }^{70}$ H. Eckhardt, L. Fritsche, and J. Noffke, J. Phys. F 14, 97, (1984).

${ }^{71}$ G. Thornton, R.F. Davis, K.A. Mills, and D.A. Shirley, Solid State Commun. 34, 87, (1980).

${ }^{72}$ G. Borstel, W. Braun, W. Neumann, and G. Seitz, Phys. Status Solidi (b) 95, $453,(1979)$.

73J. Hermanson, Solid State Commun. 22, 9, (1977).

${ }^{74}$ K. Mills, R.F. Davis, S.D. Kevan, G. Thornton, and D.A. Shirley, Phys. Rev. B 22, 581, (1980).

${ }^{75}$ P.T. Coleridge and I.M. Templeton, Phys. Rev. B 25, 7818, (1982).

${ }^{76}$ C.G. Olson, M. Piancentini, and D.W. Lynch, Phys. Rev. Lett. 33, 644, (1974).

${ }^{77}$ P. Szczpanek and R. Glosser, Solid State Commun. 15, 1425, (1974).

${ }^{78}$ N.E. Christensen and B.O. Seraphin, Phys. Rev. B 4, 3321, (1971).

${ }^{79}$ U. Starke, F. Bothe, W. Oed, and K. Heinz, Surf. Sci. 232, 56, (1990).

${ }^{80}$ J.J. Barton, C.C. Bailr, S.W. Robey, Z. Hussain, E. Umbach, and D.A. Shirley, Phys. Rev. B 34, 3807, (1986).

${ }^{81}$ J. Stoehr, R. Jaeger, and S. Brennan, Surf. Sci. 117, 503, (1982).

${ }^{82}$ R. Baudoing, Y. Gauthier, and Y. Joly, J. Phys. C 18, 4061, (1985).

${ }^{83}$ F. Jona, D. Westphal, A. Goldman, and P.M. Marcus, J. Phys. C 16, 3001, (1983). 
${ }^{84}$ F. Sette, C.T. Chen, J.E. Rowe, and P.H. Citrin, Phys. Rev. Lett. 59, 311, (1987). 